Aus der Klinik für Neurochirurgie

(Prof. Dr. med. V. Rohde)

der Medizinischen Fakultät der Universität Göttingen

\title{
Unterschiede im intensivmedizinischen \\ Management bei akuter \\ aneurysmatischer \\ Subarachnoidalblutung: \\ eine Umfragestudie
}

\author{
INAUGURAL-DISSERTATION \\ zur Erlangung des Doktorgrades \\ der Medizinischen Fakultät der
}

Georg-August-Universität zu Göttingen

vorgelegt von

Clara Charlotte Salfelder

aus

Hamburg

Göttingen 2021 
Dekan:

\section{Betreuungsausschuss}

Betreuer:

Ko-Betreuer:

\section{Prüfungskommission}

Referent:

Ko-Referentin:

Promotor:

Datum der mündlichen Prüfung:
Prof. Dr. Wolfgang Brück

PD Dr. Christian von der Brelie

Prof. Dr. José Hinz

PD Dr. Christian von der Brelie

PD Dr. Katrin Wasser

Prof. Dr. Ralf Dressel

02. November 2021 
Hiermit erkläre ich, die Dissertation mit dem Titel „Unterschiede im intensivmedizinischen Management bei akuter aneurysmatischer Subarachnoidalblutung: eine Umfragestudie“ eigenständig angefertigt und keine anderen als die von mir angegebenen Quellen und Hilfsmittel verwendet zu haben.

Göttingen, den 
Die Daten, auf denen die vorliegende Arbeit basiert, wurden teilweise publiziert:

Hernández-Durán S, Salfelder C, Schaeper J, Moerer O, Rhode V, Mielke D, von der Brelie C (2020): Mechanical ventilation, sedation and neuromonitoring of patients with aneurysmal subarachnoid hemorrhage in Germany: Results of a nationwide survey. Neurocrit Care $\underline{34}$, 236-247 


\section{Inhaltsverzeichnis}

Abbildungsverzeichnis ..................................................................................III

Tabellenverzeichnis......................................................................... IV

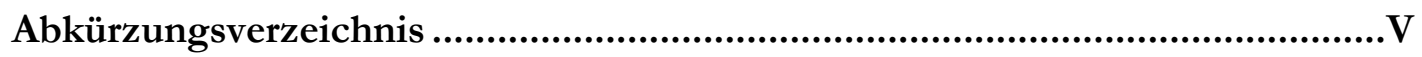

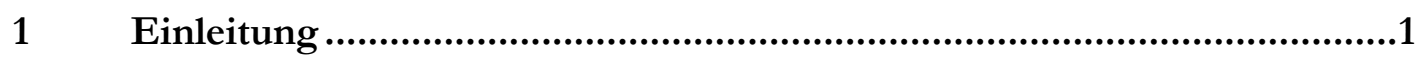

1.1 Das Krankheitsbild der akuten aneursymatischen Subarachnoidalblutung....................... 1

1.1.1 Definition und Pathophysiologie ……………………………...................................... 1

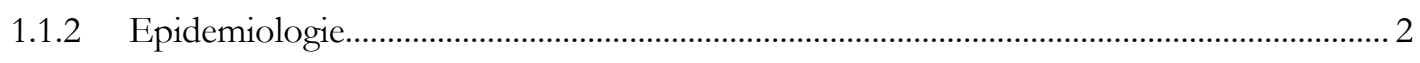

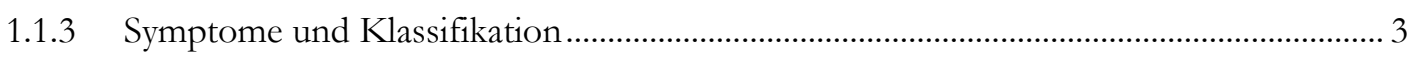

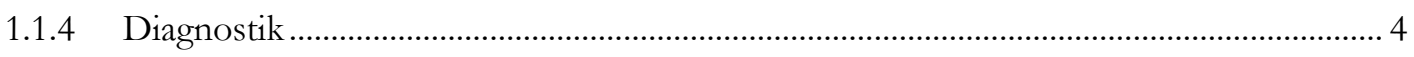

1.1.5 Therapie und klinisches Management ............................................................................ 4

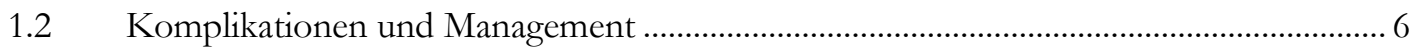

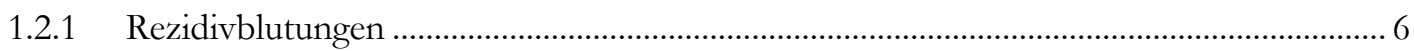

1.2.2 Zerebraler Makrovasospasmus und zerebrale Ischämie ..................................................... 7

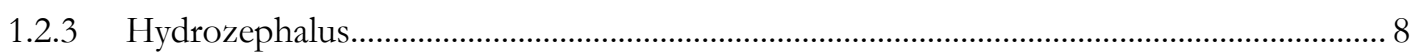

1.3 Bedeutung der Intensivtherapie bei SAB ……........................................................... 9

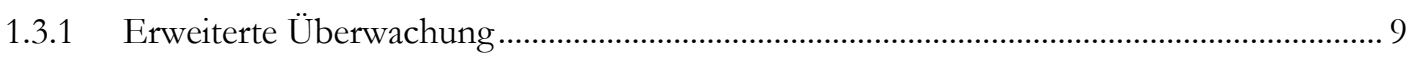

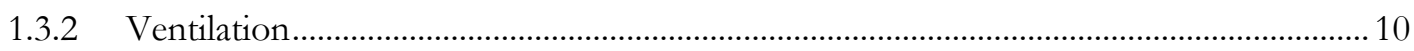

1.3.3 Analgosedierung.................................................................................................... 11

1.4 Einordnung der Arbeit im aktuellen Kontext von Leitlinen und weiteren Umfragestudien ......................................................................................................... 12

1.4.1 Spezialisierte Zentren für eine optimale Behandlung......................................................... 14

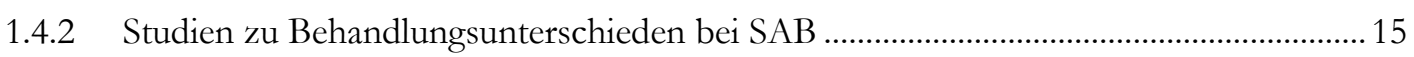

1.5 Zielsetzung der Arbeit und Leithypothese........................................................................ 16

$2 \quad$ Material und Methoden..............................................................17

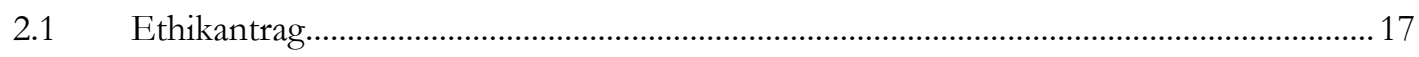

2.2 Allgemeines zur Erstellung des Fragebogens ............................................................. 17

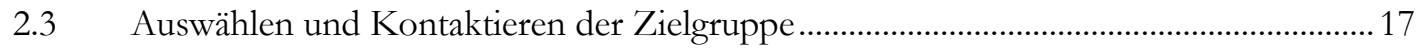

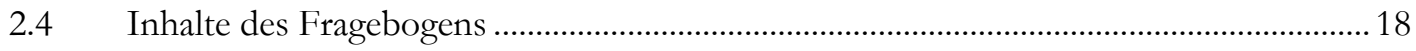

2.4.1 Allgemeine Informationen über die Struktur und Organisation des Krankenhauses... 19

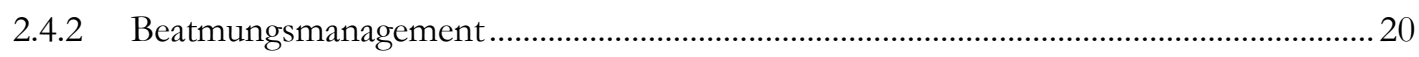

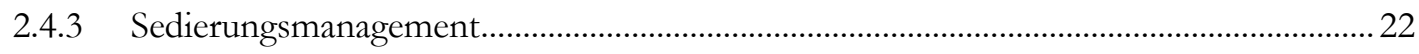

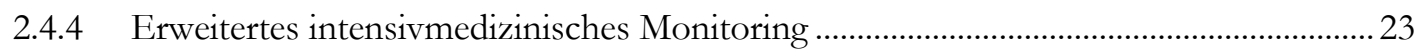

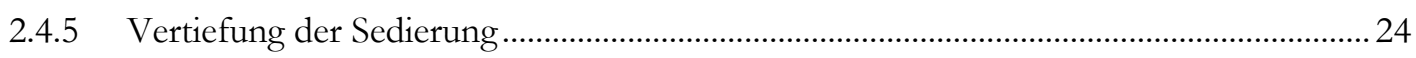

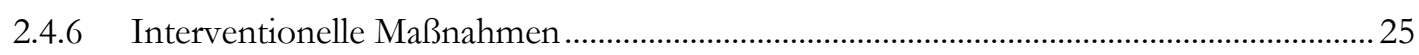

2.4.7 Reduktion der Analgosedierung und Auftreten von Komplikationen ............................2 25

$2.5 \quad$ Auswertung und Statistik ..............................................................................................2 


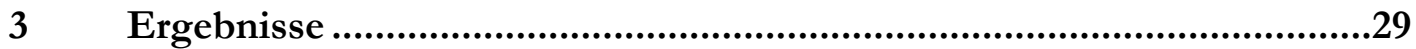

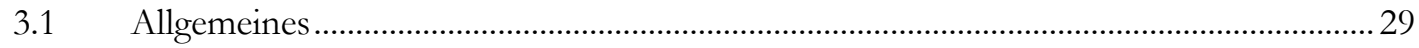

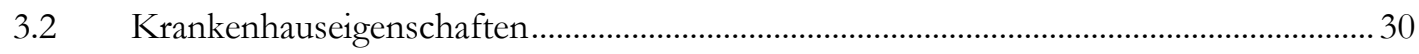

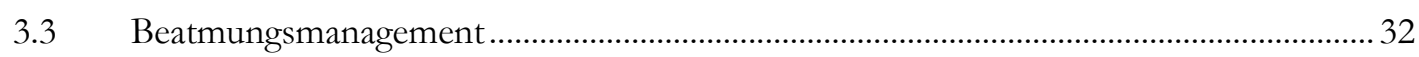

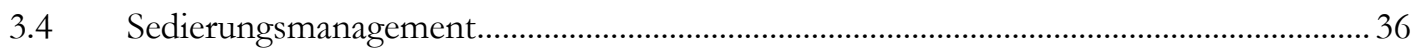

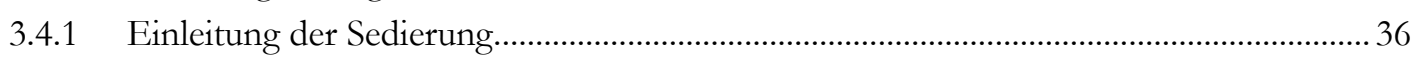

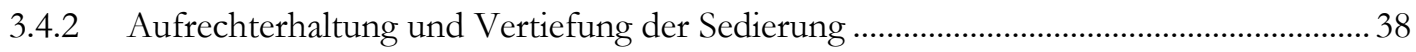

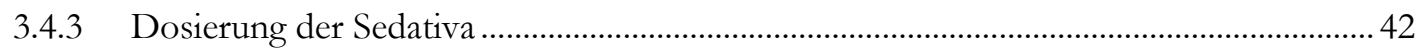

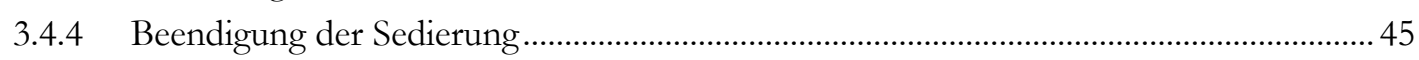

3.5 Unerwünschte Nebenwirkungen der Analgosedierung .................................................... 46

3.6 Intensivmedizinisches Monitoring ………………..................................................... 51

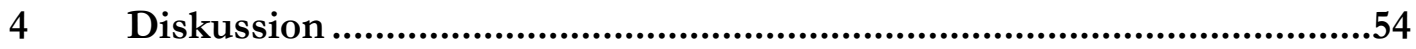

4.1 Bedeutung der Ventilations- und Sedierungstherapie bei SAB-Patienten ........................54

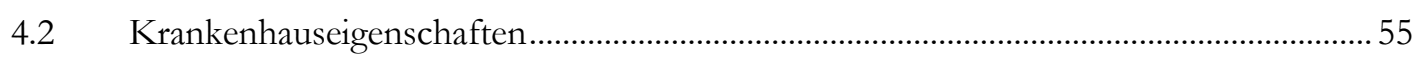

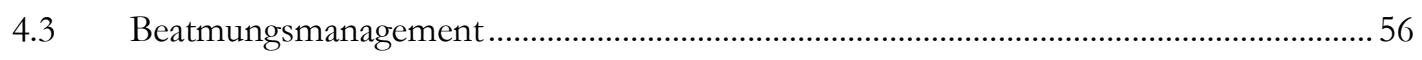

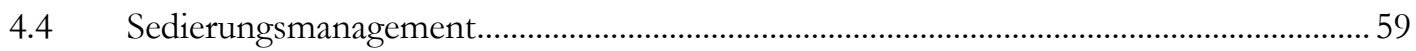

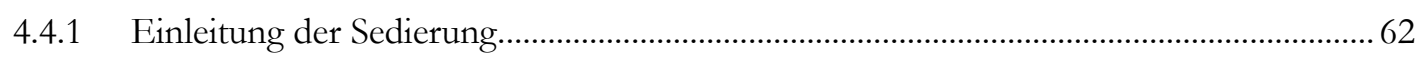

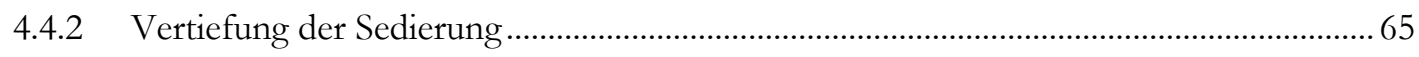

4.5 Unerwünschte Nebenwirkungen der Analgosedierung ....................................................6

4.6 Erweitertes intensivmedizinisches Monitoring ...................................................................... 69

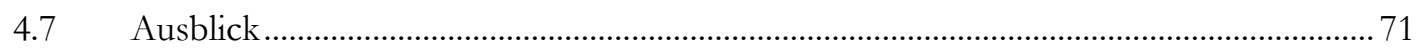

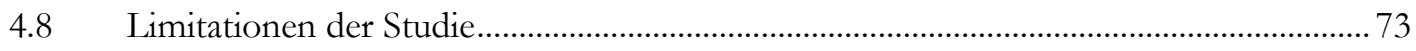

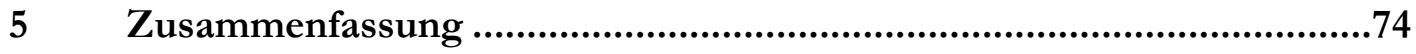

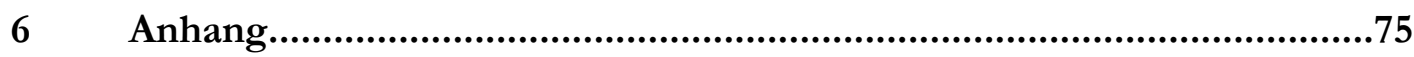

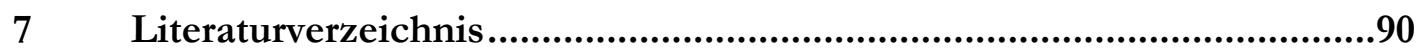




\section{Abbildungsverzeichnis}

Abbildung 1: Intraoperative Aufnahme. Clipping eines Aneurysmas I

Abbildung 2: Intraoperative Aufnahme. Clipping eines Aneurysmas II

Abbildung 3: Gesamtbehandlungsvolumen. Absolute Anzahl an SAB-Patienten pro Jahr ...... 29

Abbildung 4: Durchschnittliches Behandlungsvolumen einer Klinik von SAB-Patienten pro

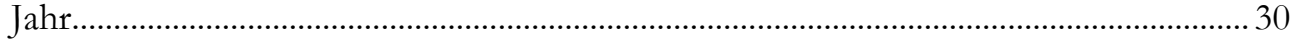

Abbildung 5: Indikationsstellung zur Beatmung...................................................................... 33

Abbildung 6: Parameter zur Indikationsstellung der mechanischen Ventilation.........................34

Abbildung 7: Medikamente zur Einleitung der Sedierung in den jeweiligen Zentren ...............37

Abbildung 8: Medikamente zur Aufrechterhaltung der Sedierung ..............................................39

Abbildung 9: Medikamente zur Vertiefung der Sedierung............................................................ 41

Abbildung 10: Variabilität der maximalen Dosierungen am Beispiel von Midazolam und

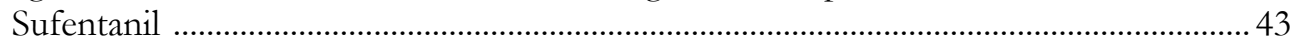

Abbildung 11: Antworten zur Klassifizierung eines Delirs........................................................47

Abbildung 12: Objektivierung des Sedierungsniveaus ...........................................................5 51

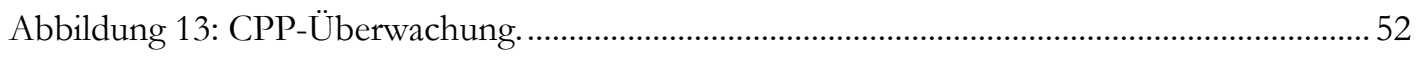




\section{Tabellenverzeichnis}

Tabelle 1: Übersicht der Leitlinien

Tabelle 2: Fragen zu allgemeinen Informationen über die Struktur und Organisation des Krankenhauses.

Tabelle 3: Fragen zum Beatmungsmanagement..................................................................... 20

Tabelle 4: Fragen zum Management der Analgosedierung ....................................................... 22

Tabelle 5: Fragen zum erweiterten intensivmedizinischen Monitoring..........................................2 23

Tabelle 6: Fragen zur Vertiefung der Sedierung........................................................................... 24

Tabelle 7: Fragen zu interventionellen Maßnahmen .............................................................. 25

Tabelle 8: Fragen zur Reduktion der Analgosedierung und dem Auftreten von Komplikationen .25

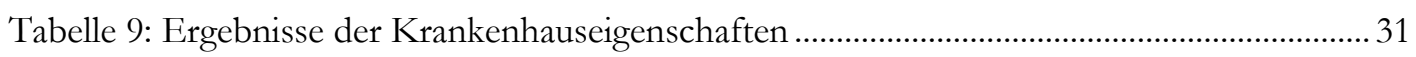

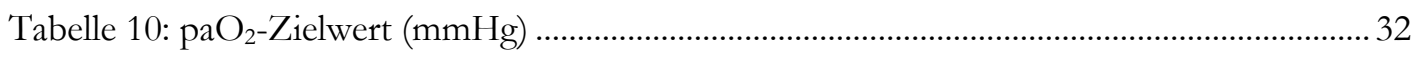

Tabelle 11: Ergebnisse zum Beatmungsmanagement ................................................................... 35

Tabelle 12: Darstellung der intubierten und analgosedierte Patienten auf der Intensivstation . 36

Tabelle 13: Medikamente zur Einleitung der Sedierung …………………………......................... 36

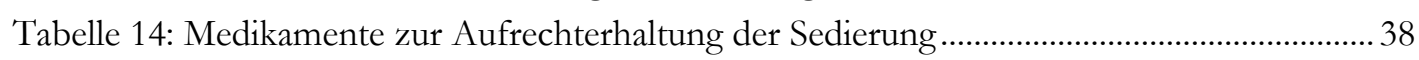

Tabelle 15: Medikamente zur Vertiefung der Sedierung.............................................................. 40

Tabelle 16: Vertiefung der Sedierung: Gabe eines Barbiturats und Dauer der Administration 41

Tabelle 17: Maximale Anzahl an verwendeten Sedativa zur Vertiefung der Sedierung............... 42

Tabelle 18: Dosierung von Midazolam und Sufentanil............................................................... 42

Tabelle 19: Einsatz von Ketamin im Rahmen der Sedierung ........................................................... 43

Tabelle 20: Einsatz von Propofol im Rahmen der Sedierung......................................................... 44

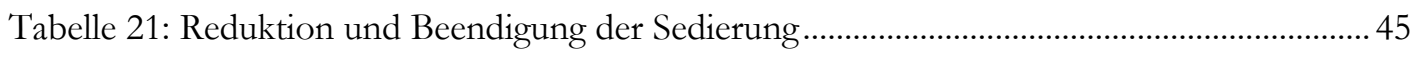

Tabelle 22: Häufigkeit des Auftretens beobachteter Komplikationen ............................................. 46

Tabelle 23: Wahrnehmung als schwere vs. leichte Komplikation.................................................... 47

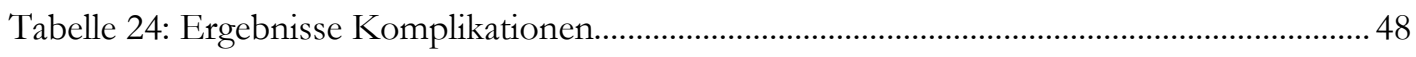

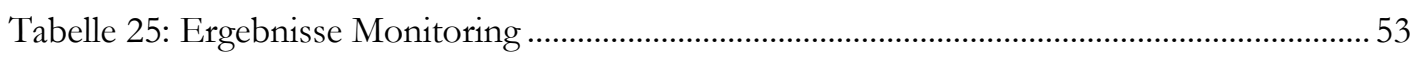

Tabelle 26: Leitlinienempfehlungen zum Beatmungsmanagement................................................5 57

Tabelle 27: Leitlinienempfehlungen zum Sedierungsmanagement ................................................. 61

Tabelle 28: Leitlinienempfehlungen zum Sedierungsmanagement ................................................66

Tabelle 29: Leitlinienempfehlungen zum Monitoring ................................................................. 71 


\section{Abkürzungsverzeichnis}

ABI Akute Hirnverletzung, acute brain injury

AnaConDa ${ }^{\circledR}$ Medizinprodukt für die Verabreichung volatiler Anästhetika, Anesthetic Conserving Device

ARDS akutes Lungenversagen, acute respiratory distress syndrome

$\mathrm{aSAB} \quad$ aneurysmatische Subarachnoidalblutung

AY Aneurysma

AWMF Arbeitsgemeinschaft der Wissenschaftlichen Medizinischen Fachgesellschaften

BIPAP biphasischer positiver Atemwegsdruck, biphasic positive airway pressure

BIS Bispektralindex

BP Blutdruck, blood pressure

CBF Zerebraler Blutfluss, cerebral blood flow

CBV Zerebrales Blutvolumen, cerebral blood volume

CIP Critical-Illness Polyneuropathie

$\mathrm{CMRO}_{2}$ zerebrale Stoffwechselrate für Sauerstoff, cerebral metabolic rate for oxygen

CPP Zerebraler Perfusionsdruck, cerebral perfusion pressure

CPPopt Optimaler zerebraler Perfusionsdruck

CSD Kortikale Streudepolarisation, cortical spreading depolarization

cCT kraniale Computertomographie

CT Computertomographie

DCI verzögert eintretende zerebrale Ischämie, delayed cerebral ischemia

DGN Deutsche Gesellschaft für Neurologie

DGNC Deutsche Gesellschaft für Neurochirurgie

DHC Dekompressive Hemikraniektomie

DIND verzögerte ischämisch-neurologische Defizite, delayed ischemic neurological deficit

DSA Digitale Substraktionsangiographie

DSI Tägliche Sedierungsunterbrechung, daily sedation interruption

ECMO extrakorporale Membranoxygenierung

EVD externe Ventrikeldrainage

GABA Gammaaminobuttersäure

GCS Glasgow Coma Scale

GHB Gammahydroxybutyrat

HVC hochvolumiges Zentrum, high-volume center

ICB intrazerebrale Blutung 
ICU Intensivstation, intensive care unit

IH Intrakranielle Hypertension

IMCCMM International Multidisciplinary Consensus Conference on Multimodality Monitoring

ISAT International Subarachnoid Aneurysma Trial

LP Lumbalpunktion

LVC niedrigvolumiges Zentrum, low-volume center

MAP mittlerer arterieller Druck, mean arterial pressure

MMCAI maligne mittlere zerebrale Arterieninfarkte, maligne middle cerebral artery infarctions

MRT Magnetresonanztomographie

NCC Neurointensivbehandlung, neurocritical care

NMDA N-Methyl-D-Aspartat

NWT Aufwachtest, neurological wake-up test

$\mathrm{paCO}_{2} \quad$ arterieller Kohlenstoffdioxidpartialdruck

$\mathrm{paO}_{2} \quad$ arterieller Sauerstoffpartialdruck

PEEP positiv endexpiratorischer Druck

PiCCO Pulskontur-Herzzeitvolumen, pulse contour cardiac output

PRIS Propofol-Infusions-Syndrom

PRx pressure reactivity index

$\mathrm{ptiO}_{2} \quad$ zerebraler Sauerstoffgewebspartialdruck, brain tissue oxygen

RAAS Richmond Agitation-Sedation Scale

ROS reaktive Sauerstoffspezies

SAB Subarachnoidalblutung

$\mathrm{SaO}_{2} \quad$ arterielle Sauerstoffsättigung

$\mathrm{StiO}_{2} \quad$ zerebrale Sauerstoffsättigung, brain tissue oxygen saturation

UH Universitätskrankenhaus, University Hospital

VAP ventilationsassoziierte Pneumonie

VST Ventilations- und Sedierungstherapie

WFNS World Federation of Neurosurgical Societies 


\section{Einleitung}

Die vorliegende Dissertationsschrift befasst sich mit den intensivmedizinischen Behandlungstrategien der akuten aneurysmatischen Subarachnoidalblutung (SAB). Die SAB ist ein nicht selten sehr schwerwiegendes und potentiell komplikationsbehaftetes Krankheitsbild, welches häufig mit schlechtem neurologischen Outcome vergesellschaftet ist.

Neben den Maßnahmen zum Verschluss des rupturierten Aneurysmas (durch z. B. operatives Clipping und endovaskuläres Coiling), sind intensivmedizinische Maßnahmen in der Behandlung der SAB von zentraler Bedeutung. Intensivmedizinsche Maßnahmen umfassen häufig eine längere Analgosedierung und eine mechanische Beatmung. Diese komplexen Therapieschritte und deren Abfolge und Anpassung müssen an den individuellen Krankheitsverlauf angepasst werden.

Das Ziel dieser Arbeit ist es, etwaige Unterschiede in der täglichen, praktischen intensivmedizinischen Behandlung zu erfassen. Hierzu wurde ein Fragebogen entwickelt, der die verschiedenen intensivmedizinischen Strategien erfasst. Die Auswertung des Fragebogens gibt daher einen Überblick über die verschiedenen Behandlungsmethoden der SAB im deutschsprachigen Raum. Ein weiterer Fokus dieser Dissertationsschrift liegt in der Einordnung der Umfrageergebnisse in den Kontext der existierenden Leitlinien.

\subsection{Das Krankheitsbild der akuten aneursymatischen Subarachnoidalblutung}

\subsubsection{Definition und Pathophysiologie}

Die SAB ist ein schwerwiegendes neurologisches Krankheitsbild. In 85\% der Fälle kommt es durch ein spontan rupturiertes Aneurysma im Bereich des Circulus arteriosus Willisii zu einer Blutung in den liquorgefüllten Subarachnoidalraum zwischen den Hirnhäuten Arachnoidea und Pia mater (Abbildung 1 und 2). In den übrigen 15\% der Fälle ensteht die SAB nicht aus einem rupturierten Aneurysma sondern z. B. als spontane perimesenzephale Blutung, als Blutung aus einer arterio-venösen Malformationen oder aus der Ruptur von dissezierten intraduralen Gefäßen (van Gijn und Rinkel 2001). Die hier vorliegende Arbeit beschäftigt sich ausschließlich mit dem Krankheitsbild der akuten aneurysmatischen SAB. 
Diese macht ca. 5\% aller Schlaganfälle aus und tritt im Vergleich zu ischämischen Schlaganfällen mit einem durchschnittlichen Lebensalter von ca. 50 Jahren deutlich früher auf (Feigin et al. 2009).

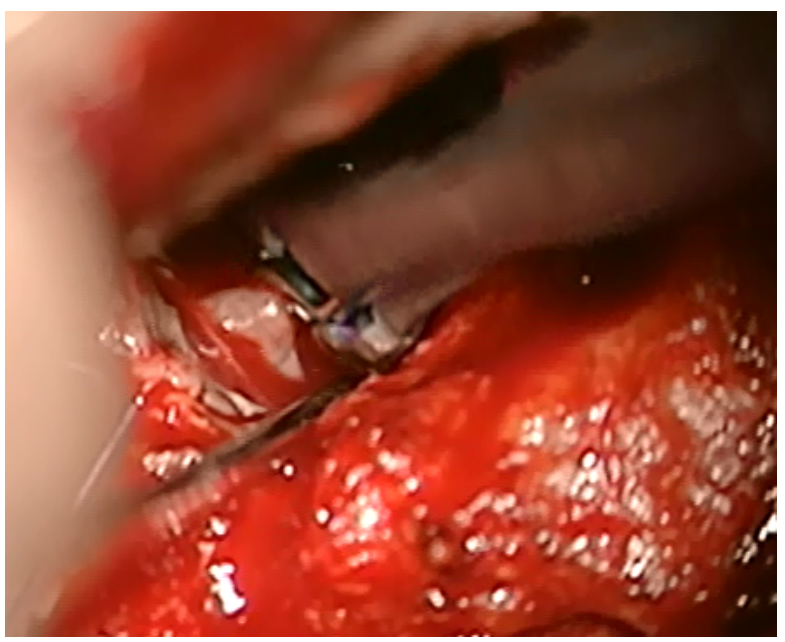

Abbildung 1: Intraoperative Aufnahme. Clipping eines Aneurysmas I. (Zur Verfügung gestellt von Frau Dr. S. Hernández-Durán, Klinik für Neurochirurgie, UMG)

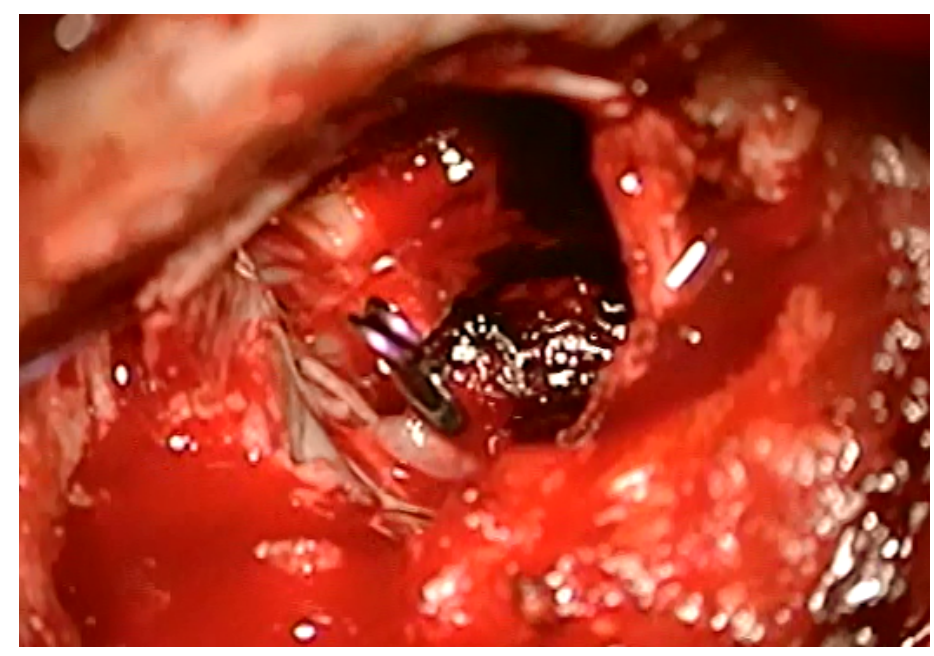

Abbildung 2: Intraoperative Aufnahme. Clipping eines Aneurysmas II. (Zur Verfügung gestellt von Frau Dr. S. Hernández-Durán, Klinik für Neurochirurgie, UMG)

\subsubsection{Epidemiologie}

Die Inzidenz der aneurysmatischen SAB beträgt ca. sechs bis neun pro 100.000 Personen/Jahr (Feigin et al. 2009; Leitlinie Subarachnoidalblutung 2012). Im Vergleich dazu kommen unrupturierte intrakranielle Aneurysmen bei ca. 3\% aller Erwachsenen vor (Rinkel et al. 1998; Vlak et al. 2011). Diese werden häufig zufällig entdeckt und erweisen sich in den meisten Fällen als asymptomatisch (Raabe et al. 2002; Wermer et al. 2007). 
Die 30-Tage-Letalität beträgt aktuell immer noch ca. 35 bis 40\% (Feigin et al. 2009; Schmutzhard et al. 2010). Bis zu 15\% aller Patienten sterben bevor sie das Krankenhaus erreicht haben. Allerdings konnte die Mortalitätsrate der SAB innerhalb der letzten Jahrzehnte stark verringert werden. Die Reduktion der Mortalitätsrate ist mit den maßgeblichen Fortschritten der einzelnen Therapiekomponenenten, den verbesserten diagnostischen Methoden sowie den engmaschig ineinandergreifenden Elemente der Rettungskette zu begründen (Hop et al. 1997; Cross et al. 2003).

Die ebenfalls sehr hohe Morbidität der SAB führt dazu, dass ein Drittel der Überlebenden eine dauerhafte Behinderung mit Pflegebedürftigkeit davonträgt, wie z. B. Einschränkungen der körperlichen Funktion. Ein Drittel der Überlebenden erleidet dauerhafte neuropsychologische Defizite, wie z. B. Störungen von Wahrnehmung und Sprache oder Persönlichkeitsveränderungen (van Gijn et al. 2007; Feigin et al. 2009; Schmutzhard et al. 2010). Nur ca. $30 \%$ der überlebenden Patienten weisen eine gute Langzeiterholung ohne psychische oder neurologische Beschwerden auf (van Gijn et al. 2007).

Entscheidend für die Prognose ist nicht nur die primäre Hirnschädigung durch die initiale $\mathrm{SAB}$, sondern auch das Auftreten möglicher SAB spezifischer Komplikationen, wie z. B. Rezidivblutungen aus dem rupturierten Aneurysma, verzögert auftretende zerebrale Ischämien und Liquorzirkulationsstörungen (Bederson et al. 2009; Schmutzhard et al. 2010). Diese Daten belegen, wie schwerwiegend eine $\mathrm{SAB}$ sein kann und wie deutlich die resultierenden Einschränkungen die Lebensqualität der Überlebenden beeinflussen können. Dies verdeutlicht den großen Stellenwert des intensivmedizinischen Managements in der Therapie der SAB.

\subsubsection{Symptome und Klassifikation}

Das Kardinalsymptom der SAB ist der plötzlich auftretende „Vernichtungskopfschmerz“. Diese Kopfschmerzen sind häufig vergesellschaftet mit neurologischen Fokalzeichen, wie beispielsweise Sehstörungen. Auch Meningismus und Symptome einer intrakraniellen Drucksteigerung, wie Vigilanzminderung, Übelkeit und Erbrechen können auftreten. Der klinische Schweregrad der SAB sollte durch die Verwendung validierter Skalen, wie z. B. Hunt and Hess oder World Federation of Neurological Surgeons (WFNS) bestimmt werden (Connolly et al. 2012). Bei der Skala nach Hunt and Hess erfolgt die Einteilung nach Schweregraden von Grad I (asymptomatisch oder leichte Kopfschmerzen) bis Grad V (tiefes Koma und moribund) (Hunt und Hess 1968). 
Die Einteilung nach WFNS orientiert sich ebenfalls an der Klinik des Patienten und hat die Glasgow Coma Scale (GCS) zur Grundlage (Teasdale und Jennett 1974).

Eine weitere Einteilung der SAB orientiert sich an radiologischen Kriterien. Die modifizierte Fisher-Skala nimmt durch die Quantifizierung der Blutmenge und der Blutverteilung in der computertomographischen Bildgebung (CT) eine Kategorisierung vor. Anhand der FisherSkala kann zum Beispiel die Wahrscheinlichkeit für das Auftreten von Vasospasmen in gewisser Weise vorhergesagt werden (Fisher et al. 1980).

\subsubsection{Diagnostik}

Insbesondere bei weniger schweren Kopfschmerzen und fehlender Symptomatik werden ca. 5-10 \% aller SAB zunächst ärztlich nicht korrekt diagnostiziert (Leitlinie Subarachnoidalblutung 2012).

Die Methode der Wahl zum Nachweis einer SAB ist die kraniale Computertomografie (cCT). Das cCT hat in den ersten 24 Stunden eine Sensitivität von ca. 95\% (Bederson et al. 2009). Die Magnetresonanztomografie (MRT) erreicht in den ersten Tagen ähnliche Werte.

Die MRT-Untersuchung hat sich im Vergleich zur CT einige Tage nach der Blutung als sensitivere Methode erwiesen (van Gijn et al. 2007). Wenn trotz unauffälliger Bildgebung der klinische Verdacht einer SAB besteht, muss eine eine Lumbalpunktion (LP) erfolgen. Die visuelle Inspektion eines etwaig gelblichen Überstands (Xantochromie) im Liquorröhrchen sollte durch einen erfahrenen Untersucher für den Ausschluss oder den Nachweis einer Xanthochromie erfolgen. Letztlich ist der spezifische Nachweis einer Ferritinmenge ausschlaggebend für die Diagnose einer SAB durch eine LP (Linn et al. 2005).

Bei Nachweis einer SAB im cCT oder einer Xanthochromie in der LP sollte eine CT-Angiographie oder digitale Substraktionsangiographie (DSA) der hirnversorgenden Gefäße zur Aneurysmadarstellung und weiteren Therapieplanung erfolgen (Leitlinie Subarachnoidalblutung 2012).

\subsubsection{Therapie und klinisches Management}

Bei der Akutversorgung der SAB ist die Sicherung der Vitalfunktionen und eine Stabilisierung des Patienten durch geeignete Basismaßnahmen das vorrangige Ziel. Danach erfolgen bildgebende Diagnostik zum Nachweis eines Aneurysmas, der Aneurysmaverschluss sowie eine erweiterte intensivmedizinische Behandlung in Abhängigkeit des Schweregrads der SAB. 


\subsubsection{Aneurysmaverschluss}

Nach der Stabilisierung des Patienten und der Diagnostik gilt es im weiteren Verlauf, das Aneurysma zu verschließen. Die Okklusion des Aneurysmas ist der zentrale therapeutische Schritt, denn hierdurch wird die Gefahr der Rezidivblutungen minimiert. Die Okklusion erfolgt entweder interventionell durch Platinspiralen (sog. Coiling) oder operativ durch einen Verschluss mit einem Metallclip (sog. Clipping). Beide Methoden wurden in einer Studie, dem International Subarachnoid Aneurysm Trial (ISAT), miteinander verglichen. In die Studie wurden SAB-Patienten in relativ gutem neurologischem Zustand (88\% mit WFNS-Grad 1-2) und mit relativ kleinen Aneurysmen eingeschlossen. Im ISAT konnte gezeigt werden, dass bei beiden Verfahren das Risiko für neuauftretende Blutungen aus dem ehemals rupturieten Aneurysma sehr gering war. Jedoch war die 5-Jahres-Mortalität bei Patienten, die durch Coiling versorgt wurden, geringfügig niedriger, als bei Patienten, die mit Clipping behandelt wurden (Molyneux et al. 2002). Eine Umfragestudie von Sakowitz et al. (2006) zur Behandlung von SAB in Deutschland kommt zu dem Ergebnis, dass die bevorzugte Behandlung von Aneurysmen der vorderen Zirkulation überwiegend chirurgisch ist, während endovaskuläre Behandlungsoptionen bevorzugt bei Aneurysmen der hinteren Zirkulation eingesetzt werden. Insgesamt wird die Modalität der Versogung mittels Clipping oder Coiling in Abhängigkeit von individuellen Faktoren getroffen, wie z. B. der Lokalisation des Aneurysmas und des Allgemeinzustands des Patienten, zudem spielt aber auch der Erfahrungsschatz der Therapeuten eine große Rolle.

\subsubsection{Basismaßnahmen zur Überwachung und Stabilisierung}

Die Basismaßnahmen im Management der SAB umfassen u. a. die Überwachung der Vitalparameter, wie Blutdruck und Herzfrequenz, sowie die arterielle Sauerstoffsättigung. Desweiteren erfolgt eine stündliche Evaluation des neurologischen Status mit Hilfe etablierter Skalen (GCS, WFNS), um fokale Defizite zu erkennen (Leitlinie Subarachnoidalblutung 2012). Zudem erfolgen EKG-Messungen und neuroprotektive Basismaßnahmen, wie die Überwachung des Elektrolythaushalts, der Köpertemperatur, der Blutzuckerwerte und des Wasserhaushalts. Bei sedierten Patienten erfolgt zusätzlich eine Überwachung des intrakraniellen Drucks (ICP). Bei der Einstellung des Blutdrucks sollte eine arterielle Hypotonie wegen der potenziell kritischen Verminderung des zerebralen Perfusionsdrucks (CPP) und der etwaig auftretenden Gefäßsspasmen zwingend verhindert werden. Allerdings muss auch der gegenteilige Zustand (Hypertonie) unbedingt vermieden werden, da es hierdurch zu einer erneuten Ruptur des Aneurysmas kommen kann. 
Es wird daher empfohlen, den arteriellen Mitteldruck (MAP) bei neurologisch unauffälligen Patienten zwischen 60 und $90 \mathrm{mmHg}$ einzustellen und systolische Blutdruckwerte über 140 mmHg zu vermeiden (Schmutzhard et al. 2010; Leitlinie Subarachnoidalblutung 2012).

Weiterhin ist eine speziell angepasste intensivmedizinische Überwachung sowie ein geeignetes Komplikationsmanagement nötig. Das erweiterte intensivmedizinischen Management umfasst u. a. bei bewusstseinseingeschränkten Patienten eine Analgosedierung und eine Intubation zur Sicherung der Atemwege mit nachfolgender maschineller Beatmung. Die Indikation zur Intubation bei reduzierter Vigilanz (GCS < 8) sollte allerdings gut abgewogen werden, da das klinisch neurologische Monitoring bei intubiert beatmeten und sedierten Patienten nicht mehr möglich ist. Auf der einen Seite gilt es eine Hypoxie und Hyperkapnie zu vermeiden, auf der anderen Seite ist der neurologische Status und das Auftreten von Komplikationen bei einem sedierten Patienten kaum mehr beurteilbar.

\subsection{Komplikationen und Management}

Die verschiedenen Komplikationen einer SAB führen häufig zu einer neurologischen und systemischen Verschlechterung der Patienten. Die Komplikationen und deren Auftreten bestimmen daher maßgeblich für das neurologische Outcome und die Mortalität. Das unmittelbare Management nach Ruptur des Aneurysmas (AY) zielt mittels Okklusion des rupturierten AYs auf das Verhindern von Nachblutungen ab. Ein weiteres Ziel ist die Stabilisierung von Atem- und Kreislaufverhältnissen. Das intensivmedizinische Management im Verlauf der Erkankung dient vor allem der Prophylaxe, Diagnostik und der ggf. notwendigen Therapie der neurologischer und systemischer Komplikationen (Seule et al. 2010). Daher werden Patienten mit einer SAB in der Regel für ca. 14 Tage intensivmedizinisch überwacht. Für die möglichen Komplikationen gibt es viele pathophysiologische Gründe, welche in den folgenden Kapiteln beschrieben werden.

\subsubsection{Rezidivblutungen}

Rezidivblutungen bei einem unbehandelten Aneurysma gehören mit ca. 40\% zu den häufigsten Komplikationen einer SAB (Coppadoro und Citerio 2011; Doukas et al. 2019). Das Risiko einer Rezidivblutung ist in den ersten Tagen am höchsten. Die Prognose ist für betroffene Patienten sehr schlecht, da ca. 80\% der Patienten an der erneuten Blutung versterben oder eine schwere Behinderung davon tragen (Dupont et al. 2010; Zhao et al. 2016; Doukas et al. 2019). 
Durch einen frühen Aneurysmaverschluss ( $<72$ Stunden) konnte allerdings die Häufigkeit von Rezidivblutungen vermindert werden (de Gans et al. 2002; Rabinstein et al. 2010).

\subsubsection{Zerebraler Makrovasospasmus und zerebrale Ischämie}

Das Autreten eines zerebralen Makrovasospasmus zählt zu den häufigsten Gründen für die neurologische Verschlechterung eines SAB-Patienten im klinischen Verlauf. Dieser führt häufig zu einer verspäteten zerebralen Ischämie (DCI) und äußert sich in 50\% der Fälle symptomatisch in einem verzögertem ischämischen Defizit (delayed ischemic neurological deficit, DIND)(Dorsch 2002; Dreier 2011). Der zerebrale Makrovasospasmus tritt in der Regel ca. 4 bis 14 Tage nach der initialen Blutung auf und betrifft ca. 30 bis 70\% der Patienten (Dorsch 2002; Sarrafzadeh et al. 2014). Die Entstehung der DCI unterliegt allerdings auch noch anderen Pathomechanismen, wie z. B. Neuroinflammation, Aktivierung von Thrombozyten und kortikaler Streudepolarisation. Diffuse Vasospasmen führen zu einer Minderdurchblutung und damit zu einer Ischämie des Hirngewebes (Macdonald 2014).

Allerdings kann die DCI auch ohne vorangegangene Vasospasmen auftreten. Ischämien können auch aus einer Phase einer vorübergehenden intrakraniellen Hypertension (IH) entstehen und durch eine Minderdurchblutung, welche durch ein zerebrales Ödem verursacht wird (Rowland et al. 2012; Macdonald 2014; Sarrafzadeh et al. 2014).

Da die DCI entscheidend zur Prognose des Patienten beiträgt, ist die Erkennung und die Prophylaxe von großer Bedeutung. Die DGN-Leitlinie Subarachnoidalblutung (2012) der Deutschen Gesellschaft für Neurologie (DGN) empfiehlt daher die Einstellung folgender protektiver Zielwerte: zentralvenöser Druck $>4 \mathrm{mmHg}, \mathrm{MAP}>70 \mathrm{mmHg}$ und $\mathrm{CPP}>60$ mmHg. Die Einnahme des Calciumantagonisten Nimodipin für drei Wochen wird von der DGN-Leitlinie Subarachnoidalblutung (2012) als einzige gesichert wirksame Primärprophylaxe von DIND nach aneurysmaler SAB empfohlen (Feigin et al. 1998; Mees et al. 2007; Leitlinie Subarachnoidalblutung 2012). Der Flüssigkeits- und Elektrolythaushalt sollte zwingend überwacht werden, um eine Hypovolämie oder eine Veränderung des Natriumhaushaltes im Sinne eines Diabetes insipidus (Hypernatriämie) oder ein zerebrales Salzverlust-Syndrom (Hyponatriämie) zu erkennen und zu behandeln (Claassen et al. 2004). Die vorbeugende Wirksamkeit der früher häufig als Kombinationsmaßnahme durchgeführten TripleH-Therapie (Hypervolämie, Hämodilution, Hypertension) zur Verbesserung der zerebralen Perfusion, konnte durch Studien bisher nicht gesichert werden (Feigin et al. 2000; Dankbaar et al. 2010; Rowland et al. 2012). 
Allerdings kann in der Akuttherapie bei bereits manifester DCI eine hämodynamische Therapie insbesondere im Sinne einer induzierten Hypertension angewendet werden (Treggiari 2011; Macdonald 2014; Leitlinie Subarachnoidalblutung 2012).

\subsubsection{Hydrozephalus}

Eine weitere, sehr schwerwiegende Komplikation stellt der akute Hydrozephalus dar. Dieser bildet sich dadurch, dass der Liquor durch die Beimischung von subarachnoidalem Blut und der gleichzeitig auftretenden intrakraniellen Drucksteigerung einen erhöhten Abflusswiderstand überwinden muss oder es durch Einbruch von Blut ins Ventrikelsystem zur Koagelbildung kommt, die im Sinne eines Okklusivhydrozephalus akut die Liquorzirkulation stört. Ein akuter Hydrozephalus tritt bei ca. 20 bis 30\% der Patienten in den ersten drei Tagen auf (Schmutzhard et al. 2010; Leitlinie Subarachnoidalblutung 2012). Durch die Erhöhung des Liquorabflusswiderstandes kann es zu einer deutlichen Steigerung des ICP kommen. Der Hydrozephalus muss zwingend zügig durch eine Liquoraußenableitung behandelt werden, damit der ICP normalisiert werden kann.

Eine Überwachung des ICP ist daher bei allen SAB-Patienten indiziert und insbesondere bei Patienten, die aufgrund der Sedierung keine Symptome eines erhöhten ICP aufweisen. Es werden Werte zwischen 15 bis 20 mmHg angestrebt (Schmutzhard et al. 2010; Leitlinie Subarachnoidalblutung 2012). Nach Empfehlung der DGN-Leitlinie Subarachnoidalblutung (2012) sollten SAB-Patienten mit einem symptomatischen oder fraglich symptomatischen Hydrozephalus eine Liquorableitung (z. B. durch eine externe Ventrikeldrainage oder eine lumbale Drainage) erhalten.

Die Liquorkommunikation kann auch im späteren Verlauf der SAB noch zu Komplikationen führen. Einen chronischen Hydrozephalus entwickeln ca. 25\% der Patienten im späteren Verlauf der Erkrankung, insbesondere diejenigen mit einer schweren SAB. Bei den Symptomen handelt es sich typischerweise um Kopfschmerzen, Erbrechen und Vigilanzminderung. Diese sollten mit einem ventrikuloperitonealen oder ventrikuloatrialen Shunt behandelt werden (Leitlinie Subarachnoidalblutung 2012). 


\subsection{Bedeutung der Intensivtherapie bei SAB}

Neben den neurologischen Komplikationen können auch systemische Komplikationen wie kardiale und pulmonale Komplikationen auftreten. Andere systemische Komplikationen sind Fieber, pulmonale Insuffizienz, akute Herzinsuffizienz, Glaskörpereinblutungen (Terson-Syndrom), Elektrolytentgleisungen und weitere internistische Krankheitsbilder. Diese nicht-neurologischen Komplikationen betonen, wie wichtig eine spezialisierte intensivmedizinische Betreuung für die betroffenen Patienten ist. Die Herausforderung für die behandelnden, interdisziplinären Ärzteteams liegt darin, neu aufgetretende Verschlechterungen der Patienten vor dem Hintergund der SAB, zu identifizieren und optimal zu behandeln. Leitlinien für die Therapie der SAB empfehlen die Behandlung auf neurochirurgisch spezialisierten Intensivstationen. Allerdings ist weder der genaue Aufbau einer solchen spezialisierten Intensivstation, noch die Ventilations- und Sedierungstherapie (VST) bei SAB genau definiert (Connolly et al. 2012; Leitlinie Subarachnoidalblutung 2012). Im Folgenden wird auf die erweiterte Überwachung, die Bedeutung der Ventilation und der Analgosedierung bei SAB im intensivmedizinischen Management eingegangen.

\subsubsection{Erweiterte Überwachung}

Um Komplikationen rechtzeitig zu erkennen und zu behandeln, sollten Patienten nach einer $\mathrm{SAB}$ auf einer Intensivstation mit erfahrenem Fachpersonal behandelt werden (Connolly et al. 2012; Leitlinie Subarachnoidalblutung 2012). Die DGN-Leitlinie Subarachnoidalblutung (2012) empfiehlt, neben den bereits erwähnten Basismaßnahmen ein engmaschiges Monitoring weiterer verschiedener Parameter, wie tägliche transkranielle Dopplersonografie und ggf. Messungen des zentralvenösen Druckes. So können beispielsweise Vasospasmen und Hypovolämie frühzeitig erkannt werden. Eine tägliche transkranielle Dopplersonografie wird empfohlen, um einen angiografischen Spasmus z. B. der Arteria cerebri media anzuzeigen. Um die regionale Perfusion und Infarkte darzustellen, werden die CT- und die MRT-Bildgebung eingesetzt. Invasives Neuromonitoring umfasst aber auch direkte Messungen des zerebralen Sauerstoffpartialdrucks (ptiO2), zerebralen Blutfluss (CBF), Temperatur und extrazellulärer Molekülkonzentrationen (Mikrodialyse) im Hirn (Chan et al. 1993; Meixensberger et al. 2001; Marion et al. 2002). 


\subsubsection{Ventilation}

Die Ventilationstherapie stellt eine elementare Säule des Managements von SAB-Patienten dar. Neurologisch stark beeinträchtigte Patienten müssen aufgrund der reduzierten Vigilanz und der fehlenden Schutzreflexe intubiert und beatmet werden. Zudem müssen Patienten mit einer erhöhten ICP-Situation analgosediert werden, auch diese Patienten müssen mit einer maschinellen Beatmung behandelt werden. Primär pulmonale Komplikationen werden bei ca. 25\% der Patienten beobachtet. Dazu zählen das kardiogene oder das neurogene Lungenödem, eine nosokomiale Pneumonie, eine Aspirationspneumonie oder eine ventilatorassoziierte Pneumonie (Friedman et al. 2003; Kahn et al. 2006). Zu einem kardialen Lungenödem kann es u. a. durch kardiale Vorerkrankungen oder als Folgen einer Vasospasmustherapie (z. B. Katecholamine und Hypervolämie) kommen. Ein Anstieg des ICP durch SAB und konsekutive Sympathikusaktivierung kann zu einem neurogenen Lungenödem führen. Das Lungenödem mit der daraus resultierenden Hypoxie steigert wiederum das Risiko für Ischämien. Somit können die neurologischen Komplikationen im Sinne eines circulus vitiosos systemische Komplikationen bedingen und andersherum. Im Falle einer respiratorischen Insuffizienz mit potentieller Hirnschädigung durch Hypoxie muss eine individuelle Riskoabwägung beim Einsatz einer mechanischen Beatmung bei Sedierung erfolgen, da eine neurologische Beurteilung dadurch deutlich erschwert wird.

Das Ziel der Beatmung ist Normoxie und Dekarboxylierung (Normokapnie). Es sollte dabei auf eine lungenprotektive Beatmungsstrategie geachtet werden (Seule et al. 2010). Es konnte gezeigt werden, dass es einen Zusammenhang zwischen Unterschieden in der Ventilation und dem Outcome der Patienten gibt (Reiff et al. 2020). An dieser Stelle sei genauer auf die Bedeutung der Einstellung des arteriellen Kohlenstoffdioxidpartialdrucks $\left(\mathrm{paCO}_{2}\right)$ hingewiesen. Sowohl Hyperkapnie als auch Hypokapnie korrelieren nachweislich mit einer schlechteren Prognose (Curley et al. 2010). Durch eine spezifische Anpassung der Ventilationsparameter und durch eine zielgerichtete Einstellung des $\mathrm{paCO}_{2}$ kann der $\mathrm{CBF}$ verbessert werden. Dieser Zusammenhang zwischen $\mathrm{CBF}$ und $\mathrm{paCO}_{2}$ stellt einen Ansatzpunkt zur Vorbeugung von Vasospasmen und ischämischen Defiziten dar (Westermaier et al. 2016). 


\subsubsection{Analgosedierung}

Eine weitere zentrale Säule des intensivmedizinischen Managements ist die Analgosedierung. Die im Rahmen der VST stattfindende Analgosedierung ist von der allgemeinen Anästhesie abzugrenzen, letztgenannte findet im Rahmen der initialen Versorgung des Aneurysmas oder bei operativen Folgeeingriffen statt. Die Indikationen für eine Sedierung umfassen beispielsweise die Reduktion von Schmerz und Ängsten, sowie die Durchführung einer mechanischen Beatmung und Blut- und Hirndruckkontrollen. Das Hauptziel der Analgosedierung besteht darin, erst Schmerzen und Beschwerden zu behandeln und dann bei Bedarf hypnotische Mittel hinzuzufügen (Mattia et al. 2006; Egerod 2009).

Allerdings ist es ein großer Nachteil, dass die Genauigkeit der klinischen Untersuchung zur Beurteilung des Zustands des Patienten und des Autretens neurologischer Komplikationen unter der Sedierung verringert wird (Samaniego et al. 2011). Daher muss die Indikation zur Sedierung individuell und streng gestellt werden, da dadurch der Goldstandard der klinischen neurologischen Untersuchung wegfällt.

Die Kenntnis der Grundlagen der molekularen Wirkweisen ist elementar für den gewinnbringensten und vorteilhaftesten Einsatz der jeweiligen Substanz zur Sedierung (Rudolph und Antkowiak 2004). Dies ist besonders wichtig bei der Sedierung eines schwererkrankten Patienten, wie beispielsweise bei der SAB. Die Unterschiede der molekularen Wirkweisen und Interaktionen der Sedativa bei intensivmedizinischen Patienten, sowie die Pathophysiologie der Grunderkrankung (z. B. SAB) werden häufig unterschätzt. Oft spielen sie eine Nebenrolle bei klinischen Entscheidungen, wenn es z. B. darum geht, ein weiteres Sedativum zur Vertiefung der Sedierung auszuwählen (Oddo et al. 2016).

In einer Arbeit von Rabinstein et al. (2010) wird die Analgesie bei SAB als ein wichtiger Aspekt der frühzeitigen medizinischen Versorgung genannt. Die genaue Durchführung der Analgesie bei SAB wird allerdings in der Arbeit von Rabinstein et al. (2010) nicht weiter definiert, dabei bleibt es im Allgemeinen bei der Erwähnung der Substanzen Paracetamol, Tramadol und Narkotika (z. B. Propofol und Ketamin). Zum Einsatz kommende Medikamentenkombinationen bei SAB sind in der Regel Sedativa (Benzodiazepine, wie z. B. Midazolam; Narkotika, wie z. B. Propofol und Ketamin) und Opioide, wie z. B. Fentanyl zur Analgesie (Smith 2007; Rowland et al. 2012).

Die Bereitstellung einer ausreichenden Analgesie und Sedierung für mechanisch beatmete, schwerkranke Patienten ist ein wichtiger Bestandteil einer optimalen Behandlung auf der Intensivstation. 
Eine tiefe Sedierung ist beispielsweise bei Hirndrucksymptomatik mit drohender Einklemmung und Hypoxie zur Reduktion des Sauerstoffverbrauchs zu indizieren (Baron et al. 2015). Es ist jedoch wichtig, die Sedierung trotz neuroprotektiver Eigenschaften kritisch zu betrachten und die Indikation streng zu stellen. Trotz der Implementierung von Sedierungsprotokollen bleibt die Anzahl von Übersedierungen hoch (Devabhakthuni et al. 2012). Der Einsatz von Sedativa kann mit erheblichen Nebenwirkungen und Risiken verbunden sein, die die mechanische Beatmung und die Verweildauer auf der Intensivstation verlängern können (Bauer et al. 1995; Nelsen et al. 2008).

\subsection{Einordnung der Arbeit im aktuellen Kontext von Leitlinen und weiteren Umfragestudien}

Die deutschsprachige S1-Leitline aus dem Jahr 2012 mit dem Titel "Subarachnoidalblutung““ der DGN, die auf der allgemein anerkannten Website der AWMF (Arbeitsgemeinschaft der Wissenschaftlichen Medizinischen Fachgesellschaften e.V., www.awmf.org) zur Verfügung gestellt wird, ist seit über fünf Jahren nicht aktualisiert worden. Die wissenschaftliche Gültigkeit dieser Leitlinie ist am 11.09.2015 abgelaufen und bis zum 29.09.2017 zum letzten Mal verlängert worden (Stand Oktober 2020). Allein dieser Umstand verdeutlicht, wie viel Aktualisierungs- und Einigungsbedarf zu diesem Thema besteht.

In den letzten Jahren wurden evidenzbasierte Leitlinien zum Management der SAB in einigen Punkten entwickelt. Diese Leitlinien legen aber den Fokus auf die Diagnostik, die mikrochirugisch/endovaskuläre Aneurysmaversorgung, Überwachungsmaßnahmen, das Komplikationsmanagement von Nachblutungen und die Erkennung und Behandlung von Vasospasmen. Es exisistieren allerdings keine Leitlinien zur Beatmungs- und Sedierungstherapie bei SAB.

Die Sedierung wird in SAB-Leitlinien nur im Zuge der Aneurysmaversorgung erwähnt und nicht im weiteren Verlauf der Nachsorge, obwohl die Sedierung gerade dort eine wesentliche Rolle spielt. Um diese Problematik zu Veranschaulichen ist im Folgenden eine Übersicht der in dieser Dissertionsschrift verwendeten Leitlinien aufgeführt (Tabelle 1). 


\section{Tabelle 1: Übersicht der Leitlinien}

Leitlinien zum Management von SAB

„Critical care management of patients following aneurysmal subarachnoid hemorrhage: recommendations from the Neurocritical Care Society's multidisciplinary consensus conference" (Diringer et al. 2011)

„Guidelines for the management of aneurysmal subarachnoid hemorrhage. American Heart Association." (Connolly et al. 2012)

„Evidence-based guidelines for the management of aneurysmal subarachnoid hemorrhage. English Edition." (Committee for Guidelines for Management of Aneurysmal Subarachnoid Hemorrhage 2012)

„S1-Leitlinie zur Subarachnoidalblutung (DGN-Leitlinie 2012)“ (Leitlinie Subarachnoidalblutung 2012)

„European stroke organization guidelines for the management of intracranial aneurysms and subarachnoid haemorrhage" (Steiner et al. 2013)

„Clinical guidelines for the emergency department evaluation of subarachnoid hemorrhage "(Meurer et al. 2016)

„Korean clinical practice guidelines for aneurysmal subarachnoid hemorrhage" (Cho et al. 2018)

Leitlinien zur Management von Intensivpatienten und anderen Kopfverletzungen

„S3-Leitlinie der Difficult Airway Society (DAS) zur Analgesie, Sedierung und Delirmanagement in der Intensivmedizin. Revision 2015 (DAS-Leitlinie 2015)“ (Baron et al. 2015)

„Guidelines for the management of severe traumatic brain injury, Fourth Edition. " (Carney et al. 2017)

Bislang werden die täglichen Kernaspekte der Intensivtherapie - die Sedierung (Medikamente und deren Dosierung) und die differenzierten Beatmungsstrategien - nicht in den Leitlinien für SAB berücksichtigt. Es werden allgemeine Standards auf SAB-Patienten angewandt, obwohl die Pathophysiologie völlig unterschiedlich ist.

Weitere Aspekte der Intensivtherapie bei SAB umfassen das Management von Komplikationen und geeignete Monitoringmaßnahmen. Diese werden bereits in separaten Empfehlungen und Leitlinien, wie z. B. in der DAS-Leitlinie zur Analgesie, Sedierung und Delirmanagement in der Intensivmedizin (Baron et al. 2015) behandelt, beziehen sich aber nicht auf die $\mathrm{SAB}$, sondern gelten generell für alle intensivmedizinischen Patienten. Jedoch stellen SABPatienten eine besondere Herausforderung dar.

Sie gleichen weder Patienten mit einem Schädelhirntrauma, noch anderen Krankheitsbildern aus denen sich klinische Applikationen bei der SAB im täglichen Gebrauch ergeben haben. 
Die Strategie der meisten neurochirurgischen Zentren besteht darin, die Behandlung der SAB-Patienten, sofern diese nicht durch SAB-Leitlinien standartisiert ist, mit den Standards und Therapieempfehlungen für traumatische Hirnverletzungen (traumatic brain injury, TBI) oder intrakraniellen Hypertension (IH) durchzuführen (Manoel et al. 2016).

Das betrifft auch die Anwendung der VST bei SAB-Patienten, die vor allem auf der Erfahrung bei der Behandlung von TBI Patienten basiert. Dies verdeutlicht eine weitere Schwierigkeit, da die pathophysiologischen Unterschiede zwischen SAB und TBI massiv sind und nicht in ausreichender Form berücksichtigt werden. Diese Situation zeigt ebenfalls den Bedarf zur Entwicklung weiterer Behandlungsalgorithmen für die SAB.

Auch stellt sich die Frage, inwiefern die verschiedenen Zentren generell Leitlinien berücksichtigen. Die klinischen Herausforderungen bei SAB-Patienten bestehen u. a. darin, dass die genaue Pathophysiologie der SAB und den assozierten neurologischen Komplikationen noch nicht vollkommen verstanden ist. Desweiteren ist die Datenlage zur VST bei SAB sehr spärlich, da die Fallzahl der SAB im Vergleich zu anderen Erkrankungen relativ gering ist. All diese Faktoren haben einen erheblichen Einfluss auf das Management der Intensivtherapie und müssen bei der VST bei SAB unbedingt berücksichtigt werden (Diringer et al. 2011).

\subsubsection{Spezialisierte Zentren für eine optimale Behandlung}

In der Leitlinie der American Heart Association (AHA) für das Management von aneurysmatischer Subarchnoidalblutung finden sich Empfehlungen zu Krankenhausmerkmalen und Versorgungssystemen (Connolly et al. 2012). Niedrigvolumige Krankenhäuser (< 10 SABFälle pro Jahr) sollten eine frühzeitige Verlegung von Patienten mit SAB in hochvolumige Zentren (> 35 SAB-Fälle pro Jahr) mit erfahrenen zerebrovaskulären Chirurgen, endovaskulären Spezialisten und multidisziplinären neurointensivtherapeutisch geschultem Personal in Betracht ziehen (Connolly et al. 2012). Diese Einteilung der Zentren in niedrig- und hochvolumige Zentren wird in dieser Arbeit übernommen. In einer amerikanische Studie wurde festgestellt, dass hochvolumige Zentren ein besseres Überleben der SAB-Patienten beobachten als niedrigvolumige Zentren (McNeill et al. 2013). Auch eine Studie von Rabinstein et. al (2010) zum multidisziplinärem Management und neuen therapeutischen Strategien bei SAB sprach dieselbe Empfehlung aus. Somit wird empfohlen SAB-Patienten in hochvolumigen und spezialisierten Zentren zu behandeln, da dort die Behandlung mit einer höheren Überlebensrate der SAB-Patienten einhergeht. 


\subsubsection{Studien zu Behandlungsunterschieden bei SAB}

Es existiert bisher eine Vielzahl an Studien zu Behandlungsunterschieden zwischen den einzelnen spezialisierten neurochirurgischen Zentren. Im Folgenden werden einige Studien und Leitlinien kurz vorgestellt, welche aufzeigen, dass Unterschiede bei der Behandlung von SAB-Patienten auftreten.

Eine große Umfragestudie von Stevens et al. (2009) analysierte die klinischen Praktiken beim Management von SAB bei Intensivmedizinern aus Nordamerika und Europa. Die beobachteten Unterschiede zwischen den Zentren in Nordamerika und in Europa, sowie zwischen hochvolumigen (> 40 SAB-Fälle pro Jahr) und niedrigvolumigen ( $\leq 40$ SAB-Fälle pro Jahr) Zentren waren besonders groß. Dabei handelte es sich um die unterschiedliche Anwendung von Clipping oder Coiling, Anfallsprophylaxe, Anwendung von Glukokortikoiden, Heparin, Einstellung ders Blutdrucks vor der Aneurysmaversorgung, und Blutzuckerkontrollen. Die Unterschiede zwischen hochvolumigen und niedrigvolumigen Zentren bestanden in der Anwendung der Triple-H-Therapie gegen Vasospasmus, in der Schnelligkeit der Aneurysmaversorgung, dem Gebrauch von Nimodipin, einer Heparinprophylaxe und kühlenden Verfahren gegen Fieber. Die Autoren der Studie schlussfolgerten, dass die Praktiken der Intensivmediziner nicht nur heterogen sind, sondern auch oft im Widerspruch zu den verfügbaren Erkenntnissen stehen (Stevens et al. 2009). Das Thema der VST wurde mit dieser Umfrage nicht untersucht.

Die Leitlinie der AHA für das Management von aneurysmatischer Subarchnoidalblutung von Connolly et. al (2012) beschreibt ebenfalls, dass es in der Literatur sehr wenig Informationen über die neurointensivmedizinische Behandlung von Patienten mit SAB gibt. Auch in dieser Leitlinie wird die VST nicht beschrieben.

Eine skandinavische Umfragestudie zu den Prinzipien der Analgosedierung und Monitoring bei Patienten mit schwerwiegender TBI und SAB in neurointensivmedizinischen Zentren zeigte große Unterschiede zwischen den befragten Institutionen (Skoglund et al. 2013). Obwohl die Gesundheitssysteme der skandinavischen Länder untereinander ähnlich sind, gab es zwischen den teilnehmenden Zentren deutliche Unterschiede bei der Wahl der Überwachungsinstrumente und Beruhigungsmittel, sowie bei der routinemäßigen Anwendung der daily sedation interruption (DSI) und neurological wake-up test (NWT). Diese Unterschiede spiegeln wahrscheinlich unterschiedliche Traditionen im klinischen Management und einen Mangel an evidenzbasierten Richtlinien wider (Skoglund et al. 2013). Die meisten Zentren überwachten routinemäßig den intrakraniellen Druck und damit indirekt den zerebralen Perfusionsdruck. 
Multimodales zerebrales Monitoring wie die sog. „Mikrodialyse“ (das Erfassen von metabolischen Parametern wie extrazelluläres Glutamat, Laktat, Pyruvat und Glyzerol), die jugularvenöse Sauerstoffsättigungsmessung im Bulbus der Vena jugularis interna (sog. „Bulbusoximetrie $\left.{ }^{"}\right)\left(\mathrm{SjvO}_{2}\right)$, und die invasive Gewebesauerstoffpartialdruckmessung in der weißen Substanz der Gehirns ( $\mathrm{ptiO}_{2}$ ) wurden nur selten eingesetzt.

Eine weitere Umfrage von Sakowitz et al. (2006) zum Management bei SAB in Deutschland zeigte ebenfalls eine große Variabilität zwischen den Kliniken, sowie ein hohes Maß an Standartisierung. Die meisten der abgefragten Aspekte bezogen sich auf den Verschluss der Aneurysmen und die Überwachung der Patienten auf der Intensivstation (Sakowitz et al. 2006). Auch in dieser Studie wurde weder das Sedierungs- und Beatmungsmanagement noch die daraus resultierenden Komplikationen abgefragt.

Die im vorausgegangenen Abschnitt erwähnten Studien und Leitlinien zeigen eine Heterogenität im klinischen Management, sowie einen Mangel an Standardisierung bezüglich der VST. Die nicht einheitliche VST bei SAB ist gleichzeitig Problem und Herausforderung. Einerseits hilft die VST beim Management von Komplikationen. Andererseits kann sie zu schwerwiegenden Komplikationen führen. Daher ist der Bedarf an Standards hoch.

\subsection{Zielsetzung der Arbeit und Leithypothese}

Die Hypothese dieser Dissertationsschrift ist, dass die Behandlungsmethoden - insbesondere die Behandlungsstrategien bei der Durchführung der VST - bei der SAB im deutschsprachigen Raum sehr unterschiedlich sind. Es wird angenommen, dass insbesondere zwischen hochvolumigen und niedrigvolumigen Zentren große Unterschiede im intensivmedizinischen Management bestehen.

Das Ziel dieser Studie ist, einen Überblick über die intensivmedizinischen Behandlungsmethoden der SAB und spezieller über die VST-Standards zu gewinnen. Ein weiteres Ziel dieser Arbeit ist es, auf die Notwendigkeit der Ausarbeitung von Leitlinien bei der VST bei SAB hinzuweisen. 


\section{Material und Methoden}

In den folgenden Abschnitten wird auf die Erstellung und Inhalte des Fragebogens, sowie auf die Datenerhebung und die Auswahlkriterien der Zielgruppe eingegangen. Eine Erläuterung der statistischen Methoden zur Auswertung der Ergebnisse schließt das Kapitel ab.

\subsection{Ethikantrag}

Im Rahmen des Projekts „Unterschiede im intensivmedizinischen Management bei akuter aneurysmatischer Subarachnoidalblutung - eine Umfragestudie“ (Antragsnummer: DOK_186_2016 vom 09.12.2016) erfolgten keine forschungsbedingten Maßnahmen am Patienten, keine Untersuchungen an menschlichem Biomaterial oder individuellen personenbezogenen Behandlungsdaten. Daher ergab sich nach Information durch die Ethikkommission keine Notwendigkeit einer Beratung.

\subsection{Allgemeines zur Erstellung des Fragebogens}

Ein interdisziplinäres Team, bestehend aus Neurochirurgen und Anästhesisten der Universitätsmedizin Göttingen, entwickelte diesen Fragebogen. Anschließend erfolgte eine praxisnahe Überprüfung von zwei unabhängigen, nicht teilnehmenden Intensivmedizinern.

Über die Website kwiksurveys ${ }^{\circledR}$ (Bristol, United Kingdom) wurde ein über das Internet abrufbarer Umfragebogen erstellt, um das intensivmedizinische Management bei SAB in verschiedenen Zentren in Deutschland zu erfassen. Der Fragebogen umfasste 73 Fragen.

Ein über die Website erstellter Link wurde via E-Mail an die Kontaktpersonen verschickt, die daurafhin den Fragebogen online ausfüllen konnten. Die Berabeitungsdauer lag bei ca. 30 min. Die Ergebnisse wurden auf der Website von kwiksurveys ${ }^{\circledR}$ gesammelt und konnten von dort weiter verarbeitet werden.

\subsection{Auswählen und Kontaktieren der Zielgruppe}

Die Zentren wurde in erster Linie nach ihrer Nennung auf der Homepage der Deutschen Gesellschaft für Neurochirurgie (DGNC) ausgewählt. Privatpraxen und ambulante Einrichtungen wurden von dieser Studie ausgeschlossen. Somit wurde sichergestellt, dass sich nur Krankenhäuser unter den teilnehmenden Zentren befanden.

Zuerst wurden die einzelnen Ärzte bzw. ihr Sekretariat telefonisch kontaktiert, hierfür wurden die angegebenen Rufnummern der Website der DGNC genutzt. Nach erfolgter 
Zustimmung wurde eine E-Mail mit einem Anschreiben und dem Freigabelink des Fragebogens zugeschickt. Der Link mit Zugang zum Fragebogen wurde per E-Mail an Intensivmediziner und Neurochirurgen an insgesamt 103 deutschen Krankenhäusern geschickt.

Der Fragebogen war für den Zeitraum vom 2. Juni 2017 bis 2. Januar 2018 online geschaltet und zur Bearbeitung verfügbar.

Nach jeweils drei und zwölf Wochen wurde eine E-Mail zur Erinnerung verschickt, insofern bis zu diesem Zeitpunkt noch keine Rückmeldung gegeben wurde. Mit Teilnahme an der Umfragestudie wurde gleichzeitig das Einverständnis zur Verwendung der Daten eingeholt. Im Januar 2018 wurde die Datenerhebung beendet und es wurde mit der Datenauswertung begonnen. Es wurde ein Rücklauf von 50 ausgefüllten Fragebögen notiert.

\subsection{Inhalte des Fragebogens}

Der Fragebogen mit insgesamt 73 Fragen umfasste sieben große Kategorien: 1) Allgemeine Informationen über die Struktur und Organisation des Krankenhauses, 2) Das Beatmungsmanagement, 3) Das Management der Analgosedierung, 4) Erweitertes intensivmedizinisches Monitoring, 5) Chirurgische Interventionen während der Behandlung der SAB, 6) Die Vertiefung der Analogsedierung, und 7) Die Reduktion der Sedierung und assoziierte Komplikationen.

Diese Aufteilung der Fragen hatte zum Ziel, das gesamte Spektrum der intensivmedizinischen Behandlung und insbesondere der Ventilationstherapie und des Sedierungsmanagements im Rahmen der interdisziplinären intensivmedizinischen Behandlung des SAB-Patienten strukturiert zu erfassen. Interventionelle Maßnahmen wie Clipping oder Coiling wurden deshalb in dieser Umfrage nicht berücksichtigt.

Im Folgenden wird auf die einzelnen Kategorien eingegangen. Die offizielle Version des online-Fragenbogens, wie er den Krankenhäusern zugestellt wurde, findet sich im Anhang. 


\subsubsection{Allgemeine Informationen über die Struktur und Organisation des Krankenhauses}

In dieser Kategorie wurden allgemeine Informationen über die Struktur und Organisation des Krankenhauses abgefragt. Dabei ging es darum, einen Überblick über die Erfahrung des Zentrums zu bekommen, um später in der Auswertung eine bessere Vergleichbarkeit der Zentren durch Gruppierungen zu ermöglichen. In Klammern gesetzt, befinden sich die jeweiligen Antwortmöglichkeiten (Tabelle 2).

Tabelle 2: Fragen zu allgemeinen Informationen über die Struktur und Organisation des Krankenhauses

\begin{tabular}{|c|c|}
\hline Fragennummer & Frage und Antwortmöglichkeit \\
\hline 1) & Name des teilnehmenden Zentrums? (Freitext) \\
\hline 2) & Wie viele Betten hat Ihr Krankenhaus? (Zahl) \\
\hline 3) & Aus welcher Stadt nehmen Sie teil? (Freitext) \\
\hline 4) & Name des Ansprechpartners? (Freitext) \\
\hline 5) & $\begin{array}{l}\text { Wie wird die Intensivstation geleitet? (rein neurochirurgisch, rein anästhesiolo- } \\
\text { gisch, gemischt, neurochirurgische/ neurologische Mitarbeit) }\end{array}$ \\
\hline 6) & $\begin{array}{l}\text { Wie häufig am Tag ist Oberarztvisite? (einmal, zweimal, dreimal, mehr als drei- } \\
\text { mal, gar nicht) }\end{array}$ \\
\hline 7) & $\begin{array}{l}\text { In welchem Schichtmodell arbeiten sie? ( } 24 \mathrm{~h} \text { Dienst, Dreischichtmodell, } 12 \mathrm{~h} \\
\text { Modell) }\end{array}$ \\
\hline 8) & Wie ist Ihr Belegschlüssel Facharzt/Assistenzarzt? $(1: 4,1: 2,2: 3)$ \\
\hline 9) & $\begin{array}{l}\text { Wie häufig finden interdisziplinäre Visiten mit z. B. Internisten, Mikrobiolo- } \\
\text { gen, etc. statt? (täglich, mehrfach wöchentlich, einmal pro Woche, nie) }\end{array}$ \\
\hline 10) & $\begin{array}{l}\text { Wie ist Ihr Behandlungsvolumen im Hinblick auf die aneurysmatische Subara- } \\
\text { chnoidalblutung zusammengefasst auf Allgemeinstationen, Intermediate Care } \\
\text { Stationen und Intensivstationen? (n/ Jahr) }\end{array}$ \\
\hline 11) & $\begin{array}{l}\text { Wie viele Patienten mit SAB behandeln Sie in Ihrem Zentrum geschätzt pro- } \\
\text { zentual auf der Intensivstation? (Anzahl/ Jahr) }\end{array}$ \\
\hline
\end{tabular}




\subsubsection{Beatmungsmanagement}

Dieser Abschnitt beinhaltet 13 Fragen zur Vorgehensweise bei der Beatmungstherapie. Dabei wurden vor allem Fragen nach der Verwendung und dem Einsatz objektivierbarer Kriterien zur Betamungsindikation (modified Fisher Skala, WFNS-Grad) gestellt. Es wurde erfragt, inwieweit weitere objektive Kriterien wie Horowitz-Index, zerebraler Vasospasmus, sowie messbare kardiale Mitbeteiligung eine Indikation zur Beatmung darstellten. Ein anderer Schwerpunkt dieser Kategorie befasste sich mit genauen Nachfragen zu den gewünschten lokalen Einstellungen von $\mathrm{paO}_{2}$, sowie $\mathrm{paCO}_{2}$ und den jeweiligen Zentrumsstandards für die differenzierten Beatmungseinstellungen. Es folgt untenstehend die Auflistung der Fragen (Tabelle 3).

Tabelle 3: Fragen zum Beatmungsmanagement

\section{Fragennummer Frage und Antwortmöglichkeit}

Wie viele der SAB-Patienten sind intubiert/beatmet und analgosediert? (< $10 \%, 10-20 \%, 20-30 \%, 30-40 \%, 40-50 \%,<50 \%, 50-60 \%, 70-80 \%,>80 \%)$

Gibt es bei Ihnen eine Indikation zur maschinellen Beatmung bei SAB modified Fisher 3 oder SAB mit begleitender ICB/VEB (modified Fisher 4)? (Ja/Nein)

Bei Indikation zur maschinellen Beatmung aufgrund der reduzierten Vigilanz/WFNS-Grad: ab welchem Schwellenwert wird das bei Ihnen regelmäBig/standartisiert durchgeführt? (Grad 1, Grad 2, Grad 3, Grad 4, Grad 5, WFNS-Grad keine formale Indikation)

Bei Indikation zur maschinellen Beatmung: Pulmonaler Gasaustausch, Aspiration: Wird bei Ihnen der Horowitz-Index in die Bewertung der Indikation zur Beatmung miteinbezogen? (Ja/Nein)

Bei Indikation zur maschinellen Beatmung: Pulmonaler Gasaustausch, Aspiration: Ab welchem Horowitz-Index wird bei Ihnen die Indikation zur maschinellen invasiven Beatmung gestellt? $(<200,<100)$

Indikation zur maschinellen Beatmung: Ist ein zerebraler Vasospasmus, welcher in der initialen Angiographie diagnonstiziert wird, bei Ihnen ein Grund für eine maschinelle Beatmung/Sedierungsperiode? (Ja/Nein)

Indikation zur maschinellen Beatmung: Stellen Sie eine Indikation zur maschinellen Beatmung und Analgosedierung, falls die SAB durch eine signifikante kardiale Mitbeteiligung verkompliziert wird? (Ja/Nein/Je nach Ausmaß der kardialen Mitbeteiligung gemessen mit z. B. PiCCO Katheter oder Echokardiographie)

Wenn die Indikation zur Beatmung gestellt wird - wie hoch ist der angestrebte $\mathrm{paO}_{2}(\mathrm{mmHg})$ ? $\left(<80,80-100,>100\right.$, Steuerung nach $\left.\mathrm{SaO}_{2}\right)$ 


\section{Fragennummer Frage und Antwortmöglichkeit}

20)

Wenn die Indikation zur Beatmung gestellt wird - wie hoch ist der angestrebte $\mathrm{paCO}_{2}(\mathrm{mmHg})$ ? $(<35,35-45,>45)$

21) Sonstige Indikationen zur maschinellen Beatmung bei SAB-Patienten: (Freitext)

22) Gibt es in Ihrer Klinik einen Optimum $\mathrm{paO}_{2}$ auf den die Beatmungseinstellungen angepasst werden? (Ja/Nein/Falls ja, wie ist der Wert?)

Gibt es in Ihrer Klinik einen Optimum $\mathrm{paCO}_{2}$ auf den die Beatmungseinstellungen angepasst werden? (Ja/Nein/Falls ja, wie ist der Wert?)

Welches kontrollierte maschinelle Beatmungsverfahren ist auf Ihrer Intensivstation das Standardbeatmungsverfahren bei sedierten Patienten? (Druckkontrollierte Beatmung mit Möglichkeit der Spontanatmung auf oberem und unterem Druckniveau/BIPAP (biphasic positive airways pressure), Volumenkontrollierte Beatmung, „Klassische“ Druckkontrollierte Beatmung)

Horowitz-Index $=$ arterieller Sauerstoffpartialdruck $\left(\mathrm{paO}_{2}\right) / \mathrm{O}_{2}$-Fraktion der Atemluft 


\subsubsection{Sedierungsmanagement}

Dieser Abschnitt umfasst zehn Fragen zur Vorgehensweise bezüglich der Analgosedierung bei SAB-Patienten (Tabelle 4). Hierbei wurden die zum Einsatz kommenden Medikamente zur Induktion und die jeweiligen Dosierungen abgefragt. Weiterhin wurden Angaben zu den Verfahren zur Objektivierung der Sedierungstiefe erhoben, wie z. B. Bispectralindex, RASSScore, Narcotrend ${ }^{\circledR}$ oder der ICP. Ein weiterer Fokus liegt auf der Messung und Stellenwert des CPP und den angestrebten Zielwerten.

Tabelle 4: Fragen zum Management der Analgosedierung

\section{Fragennummer Frage und Antwortmöglichkeit}

25)

Welche Kombination aus Medikamenten ist Mittel der Wahl zur Induktion/Einleitung von Sedierung bei SAB-Patienten? (Ketamin, Benzodiazepin, Propofol, immer Kombination mit Opioiden, immer Kombination mit Muskelrelaxans) Wird ein Benzodiazepin zur Aufrechterhaltung verwendet? (Nein/Ja/Midazolam/Diazepam)

Welche Kombination aus i.v. Sedativa wird in Ihrer Klinik standardisiert bei Indikation zur Aufrechterhaltung der Analgosedierung zuerst verabreicht? (Freitext)

Wird ein Opioid verwendet? (Nein/Ja/Remifentanil/Fentanyl/Sufentanil)

Welche Dosierung des Benzodiazepins ist die maximale Dosierung pro Stunde bei einem normgewichtigen Erwachsenen? (mg/h)

Welche Dosierung des Opioids ist die maximale Dosierung pro Stunde bei einem normgewichtigen Erwachsenen? $(\mu \mathrm{g}(\mathrm{mg}) / \mathrm{h})$

Welches Verfahren zur Objektivierung der Sedierungstiefe verwenden Sie? (Bispektralindex, RASS-Score, Narcotrend ${ }^{\circledR}, \mathrm{ICP}$ )

Monitoring der Sedierungstiefe: wie wird in Ihrer Klinik der intrakranielle Druck (ICP) gemessen? (ICP wird nicht gemessen, über ICP Sonde, über Externe Ventrikeldrainage, andere druckregulierte Drainagesysteme)

Verwenden Sie in Ihrer Klinik ein optimum cerebral perfusion pressure (CPPopt) gesteuertes Blutdruckmanagement in der Intensivtherapie bei SAB-Patienten? (Ja/Nein) 


\subsubsection{Erweitertes intensivmedizinisches Monitoring}

Dieser Abschnitt schließt sich inhaltlich dem Vorherigen an und erhebt drei weitere, mehr in die Tiefe gehende Fragen bezüglich des erweiterten Monitorings (Tabelle 5). Das besondere Interesse liegt hierbei auf der Erhebung der Vitalparameter, wie z. B. die Pulsüberwachung durch PiCCO oder die Messung des $\mathrm{ptiO}_{2}$, sowie auf der Diagnosestellung einer insuffizienten Sedierung.

Tabelle 5: Fragen zum erweiterten intensivmedizinischen Monitoring

\section{Fragennummer Frage und Antwortmöglichkeit in Klammern dahinter}

Gibt es eine regelhafte Pulskonturanalyse und Thermodilution (PiCCO-Monitoring), Pulmonalarterienkatheter oder intermittierende Echokardiographie bei den sedierten SAB-Patienten? (Ja/Nein)

Findet in der Standardbehandlung von intubiert beatmeten, analgosedierten SAB-Patienten in Ihrer Klinik regelmäßig eine Messung des zerebralen Sauerstoffpartialdrucks (ptiO2) statt? (Ja/Nein)

Wie wird nach dem Ausschluss einer behandelbaren ICP-Erhöhung (z. B. Liquordrainage erhöhen, intrazerebrales Hämatom entfernen, zusätzliche EVD (externe Ventrikeldrainage), Anpassung der Lagerung, Anpassung der Beatmung/Normokapnie, Antiepileptika, Normothermie etc.) die Diagnose einer möglicherweise insuffizienten Sedierung gestellt? (klinisch, anhand von elektrophysiologischem Monitoring (BIS/Narcotrend $®)$, anhand des ICP) 


\subsubsection{Vertiefung der Sedierung}

Dieser Abschnitt behandelt zwölf Fragen zur Vertiefung und Aufrechterhaltung der medikamentösen sowie inhalativen Sedierung (Tabelle 6). Im Speziellen wurde nach einzelnen Medikamenten und der jeweiligen Dosierung gefragt, sowie nach beobachteten Komplikationen, die im klaren Zusammenhang mit den Sedativa gesehen werden.

Tabelle 6: Fragen zur Vertiefung der Sedierung

\section{Fragennummer Frage und Antwortmöglichkeit}

Welches Medikament ist bei SAB-Patienten in Ihrer Klinik das Mittel der Wahl zur Vertiefung der Sedierung? (Gammahydroxybutyrat, Clonidin, Ketamin, Propofol, anderes Benzodiazepin, anderes Opioid)

Wie viele Sedativa werden maximal eingesetzt? $(1,2,>3)$

Welche weiteren Medikamente zur Vertiefung der Analgosedativa werden regelmäßig eingesetzt? (Gammahydroxybutyrat, Clonidin, Ketamin, Propofol, anderes Benzodiazepin, anderes Opioid)

Wenn Propofol: Welche Dosierung des Propofols ist die maximale Dosierung pro Stunde bei einem normgewichtigen Erwachsenen? $(\mathrm{mg} / \mathrm{h}$ )

Wie oft sehen Sie Komplikationen im Zusammenhang mit der Propofolgabe? (sehr selten $<1 \%$, selten zwischen 1-10\%, häufig $>10 \%$ )

Welche Komplikationen mit Propofol treten auf? (Freitext)

Wie lange wird Propofol gegeben? (maximal 3 Tage, maximal 7 Tage, länger als 7 Tage)

Wenn Ketamin: Welche Dosierung des Ketamins ist die maximale Dosierung pro Stunde bei einem normgewichtigen Erwachsenen? (mg/h)

Wenn Ketamin: verwenden Sie bei Ketamin Sedierung regelhaft ein erweitertes kardiales Monitoring ((Pulskonturanalyse und Thermodilution (PiCCO-Monitoring), Pulmonalarterienkatheter oder intermittierende Echokardiographie) z. B. aufgrund der bekannten negativen Inotropie...)? (Ja/Nein)

(Ja/Nein)

Wie werden Barbiturate engesetzt? (kurzzeitig, langzeitig, kein Einsatz)

Wird bei Ihnen inhalative Sedierung (z. B. mit AnaConDa ${ }^{\circledR}$ ) benutzt? (Ja/Nein/ Es ist mir nicht bekannt, dass inhalative Sedierung als Möglichkeit zur Sedierung bei SAB-Patienten eingesetzt werden kann)

50) Wie sind Ihre Erfahrung mit inhalativer Sedierung bei SAB-Patienten? (überwiegend gut, überwiegend schlecht, risikoreiches Verfahren, keine Erfahrung) 


\subsubsection{Interventionelle Maßnahmen}

Dieser Abschnitt mit zwei Fragen befasst sich mit den interventionellen Maßnahmen der Hemikraniektomie sowie der Tracheostomie (Tabelle 7).

Tabelle 7: Fragen zu interventionellen Maßnahmen

\begin{tabular}{ll}
\hline \hline Fragennummer & Frage und Antwortmöglichkeit \\
\hline 51$)$ & $\begin{array}{l}\text { Was gilt als hausinterne Schwelle zur dekompressiven Hemikraniektomie bei } \\
\text { SAB-Patienten? (Freitext) }\end{array}$ \\
& $\begin{array}{l}\text { Wann wird in Ihrer Klinik ein langzeitbeatmeter SAB Patient tracheostomiert? } \\
\text { (früh }<3 \text { Tage, zwischen 3-10 Tagen, eher spät }>10 \text { Tage) }\end{array}$ \\
\hline \hline
\end{tabular}

\subsubsection{Reduktion der Analgosedierung und Auftreten von Komplikationen}

Der letzte Abschnitt mit 20 Fragen zur Reduktion und Beendigung der Sedierung, behandelt ebenso die mit der Sedierung assozierten Komplikationen, und ob diese als leicht oder als schwer gesehen werden. Diese Fragen wurden weiterhin nach Sedierungstiefe und Sedierungslänge unterteilt (Tabelle 8 ).

Tabelle 8: Fragen zur Reduktion der Analgosedierung und dem Auftreten von Komplikationen

\begin{tabular}{ll}
\hline \hline Fragennummer & Frage und Antwortmöglichkeit \\
\hline 53$)$ & In welchen Reduktionsschritten wird bei Ihnen die Sedierung reduziert? (\% der \\
& Höchstdosis) \\
& Was wird als leichte Sedativa-assoziierte Nebenwirkung/Komplikation wahr- \\
& genommen? (leichte Obsitpation, schwere Obstipationen, Ileus, tiefe Beinven- \\
& enthrombose, Lungenembolie, ventilatorassoziierte Pneumonie, milde Erhö- \\
& hung der Transaminasen, milde Erhöhung der Cholestaseparameter, generali- \\
& sierte Muskelatrophie, Critical-Illness-Polyneuropathie, Delir, Katecholamin- \\
& assoziierte Nekrosen, Dekubitus, Sepsis) \\
& Was wird als schwere Sedativa-assoziierte Nebenwirkung/Komplikation wahr- \\
& genommen? (leichte Obstipation, schwere Obstipationen, Ileus, tiefe Beinven- \\
& enthrombose, Lungenembolie, ventilatorassoziierte Pneumonie, Erhöhung der \\
& Transaminasen, Erhöhung der Cholestaseparameter, generalisierte Mus- \\
& kelatrophie, Critical-Illness-Polyneuropathie, Delir, Katecholamin-assoziierte \\
& Nekrosen, Dekubitus, Sepsis) \\
& Sedativa-assoziierte Komplikationen sehen wir bei SAB-Patienten in unserer \\
& Klinik... (sehr selten < 1\%, selten 1-10\%, häufig > 10\%)
\end{tabular}




\section{Fragennummer Frage und Antwortmöglichkeit}

57)

Wie viele ventilatorassoziierte Pneumonien gab es bei Ihnen bei den letzten 30 analgosedierten SAB-Patienten (alternativ bei den intubiert beatmeten, analgosedierten SAB-Patienten im Jahr 2016)? (< 10, 10-20, 21-30, 31-40)

Kommt bei Ihnen eine extensivierte medikamentöse Stimulation der MagenDarm-Passage aufgrund von Sedativa - induzierter Darmmotilitätsstörung zum Einsatz? (Ja, immer/ Ja, bei gestörter Darmmotilität/ Nie)

Wie viele Laparotomien (z. B. aufgrund von Ileus - Problematik oder Obstipation) gab es bei Ihnen bei den letzten 30 analgosedierten SAB-Patienten? (wenige $<5,5-10,>10$ ) Wie viele sedierungsassoziierte Lungenembolien gab es bei Ihnen bei den letzten 30 analgosedierten SAB-Patienten? ( $<5$ von 30, $>5$ von 30, $>10$ von 30)

Wie viele sedierungsassoziierte Transaminase-Erhöhungen/Cholestaseparameter - Erhöhungen etc. gab es bei Ihnen bei den letzten 30 analgosedierten SAB-Patienten? ( $<5$ von $30,>5$ von $30,>10$ von 30$)$

Wie häufig sehen Sie in Ihrer Klinik hämodynamische Komplikationen von Sedierungstherapie wie z. B. einen zügigen Blutdruckabfall? (\% der intubiert beatmeten SAB-Patienten) $(<10 \%, 10-50 \%,>50 \%)$

Können Sie sich an eine drastische Komplikation erinnern, die durch eine zu tiefe Sedierung hervorgerufen worden ist? (Ja/Nein)

Um was für eine Komplikation handelte es sich? (Freitext)

Mit welchem Medikament war diese assoziiert? (Freitext)

Wie lange liegt diese zurück? ( $<1$ Monat, 2-3 Monate, 4-5 Monate, > 6 Monate, $>1 \mathrm{Jahr}, \quad>2$ Jahre)

Hätte man diese Komplikation vermeiden können? Wenn ja, wodurch? (Freitext)

Können Sie sich an eine drastische Komplikation erinnern, die durch eine zu lange Sedierung hervorgerufen worden ist? (Ja/Nein)

Um was für eine Komplikation handelte es sich? (Freitext)

Mit welchem Medikament war diese assoziiert? (Freitext)

Wie lange liegt diese zurück? (<1 Monat, 2-3 Monate, 4-5 Monate, > 6 Monate, $>1 \mathrm{Jahr}, \quad>2$ Jahre)

Hätte man diese Komplikation vermeiden können? Wenn ja, wodurch? (Freitext) 


\subsection{Auswertung und Statistik}

Zunächst wurden die Zentren je nach lokaler Behandlungs-Fallzahl kategorisiert. Diese Einteilung der Zentren wurde gewählt, da angenommen wurde, dass eine ähnliche Fallzahl an SAB-Patienten für eine ähnliche technische Ausstattung und Expertise der Zentren spricht. Folgende Kategorien wurden vergeben:

a) akademische/Universitätskrankenhäuser (university hospital, $\mathrm{UH}$ ),

b) nicht-akademische hochvolumige Zentren (bigh volume center, HVC) mit $\geq 30$ SAB-Patienten/Jahr,

c) nicht-akademische niedrigvolumige Zentren (low volume center, LVC) mit $<30$ SAB-Patienten/Jahr.

Eine Einteilung in weitere Subgruppen erfolgte zwischen denjenigen Intensivstationen (ICU) unter der Leitung von Neurologen und/oder Neurochirurgen (Neuro-ICU), und denjenigen interdisziplinären ICUs, welche unter der gemeinsamen Leitung von Intensivmedizinern, Neurochirurgen und anderen Spezialisten nicht-neurologischer Fachrichtungen (MixedICU) stehen. Diese Einteilung wurde in der Annahme gewählt, dass sich die Behandlung in Abhängigkeit der Expertise der beteiligten Fachrichtungen unterscheiden könnte.

Um die Daten des Fragebogens mit den aktuellen Standardbehandlungsvorschlägen der Intensivmedizin bewerten und vergleichen zu können, wurden allgemeine nationale und internationale Leitlinien für die Behandlung von SAB-Patienten, sowie Richtlinien, die sich auf die einzelnen Punkte des Fragebogens konzentrieren, analysiert und für einen Vergleich vorbereitet. Zu diesem Zweck wurde in PubMed eine Literaturrecherche durchgeführt. Die verwendeten Suchbegriffe waren u. a.: „subarachnoid hemorrhage, guidelines subarachnoid hemorrbage, intensive care management, sedation management, ventilation management".

Die Antworten der Zentren als zu vergleichende Parameter wurden in Kreuztabellen zusammengefasst. Die Häufigkeitsverteilung der jeweiligen Antworten als Vergleichsstatistik wurde aus diesen Kreuztabellen ermittelt. Die Ergebnisse wurden auf ihre Unabhängigkeit in Bezug auf die Gruppeneinteilung der Zentren mit Hilfe des Pearsons Chi Quadrat Test auf ihre statistischen Signifikanz hin überprüft. Die statistische Auswertung erfolgte mit der Statistiksoftware SPSS ${ }^{\circledR}$ v.25 (IBM ${ }^{\circledR}$, Armonk, New York, United States).

Eine statistische Signifikanz wurde bei einem $\mathrm{p}$-Wert weniger/gleich als $0,05(\mathrm{p} \leq 0,05)$ angenommen. Als Nullhypothese wurde angenommen, dass große Unterschiede zwischen den Antworten der jeweiligen Zentren zu erwarten sind. 
Im Rahmen der Auswertung wurde die Möglichkeit einer Bonferroni-Korrektur erwogen, um der Alphafehlerkumulierung entgegenzuwirken und somit falsch positive p-Werte auszuschließen. Allerdings wurde von dieser Methode Abstand genommen, da bei der hohen Zahl an errechneten p-Werten, nach der Analyse kein p-Wert mehr als signifkant anzunehmen gewesen wäre. Es wäre zur Annahme von falsch negativen p-Werten gekommen. Von einer Clusteranalyse über die gesamte Datenmenge wurde im Laufe des Auswertungsprozesses ebenfalls Abstand genommen. Zusammen mit dem Institut für medizinische Statistik der Universtität Göttingen wurde untersucht, ob anhand von Antwortmustern neue Untergruppen identifiziert werden konnten, die nicht mit der ursprünglichen Einteilung nach Fallzahl im Zusammenhang standen. Allerdings zeigte sich dieser statistische Ansatz als nicht zielführend und bei der heterogenen Datenmenge auch als technisch nicht realisierbar. Die Analyse führte zu keiner medizinisch-inhaltlich zielführenden Aussage und wurde deshalb verworfen. Zur besseren Veranschaulichung der Daten wurden Diagramme mit Microsoft ${ }^{\circledR}$ Excel (für Mac v.16.17), sowie SPSS ${ }^{\circ}$ v.25 (IBM®, Armonk, New York, United States) erstellt. Es wurden hauptsächlich Balkendiagramme zur übersichtlichen Darstellung von Häufigkeiten verwendet. Die statistische Auswertung erfolgte nach Beratung durch das Institut für medizinische Statistik der Universität Göttingen. 


\section{Ergebnisse}

Von den insgesamt 103 Zentren, welche kontaktiert und um Teilnahme an der Umfragestudie gebeten wurden, antworteten 50 Zentren (Antwortrate von 49\% ). Aufgrund der Länge und des Umfangs des Fragebogens werden in dieser Dissertationsschrift nur die wesentlichen Ergebnisse präsentiert und diskutiert. Zur besseren Übersichtlichkeit wurden diese in den einzelnen Absätzen zusammengefasst. Um alle 73 Fragen einzusehen, sei an dieser Stelle auf den Anhang bzw. den Material- und Methoden- Teil verwiesen. Da es nicht verpflichtend war, alle Fragen zu beantworten, weisen einige Fragen eine niedrigere Antwortrate auf. Dieser Umstand wurde bei der Analyse berücksichtigt und die jeweilige Antwortrate wurde angeben.

\subsection{Allgemeines}

Die teilnehmenden Kliniken wurden in drei Gruppen aufgeteilt: 21 ( $n=21 / 50,42 \%)$ Universitätskliniken (Universitätskrankenhaus, $\mathrm{UH}$ ), 23 ( $\mathrm{n}=23 / 50,46 \%$ ) Maximalversorger (hochvolumige Zentren mit $\geq 30 \mathrm{SAB} / \mathrm{Jahr}, \mathrm{HVC})$, und 6 ( $\mathrm{n}=6 / 50,12 \%)$ Krankenhäuser, die als niedrigvolumige Zentren kategorisiert wurden ( $<30 \mathrm{SAB}-$ Patienten /Jahr, LVC).

Es wurde eine Inzidenz von SAB von 7,5/100.000 pro Jahr angenommen und die Häufigkeit der Behandlung von SAB in den jeweiligen Zentren erfasst. Die Antworten der teilgenommenen Kliniken bilden somit $58 \%(n=3494 / 6000)$ des Behandlungsvolumens von SAB in Deutschland ab (Abbildung 3 und Abbildung 4). Die Kategorie der UH bildet die Gruppe, die am meisten SAB-Fälle/Jahr angibt, dicht gefolgt von den HVC und mit größerem Abstand LVC.

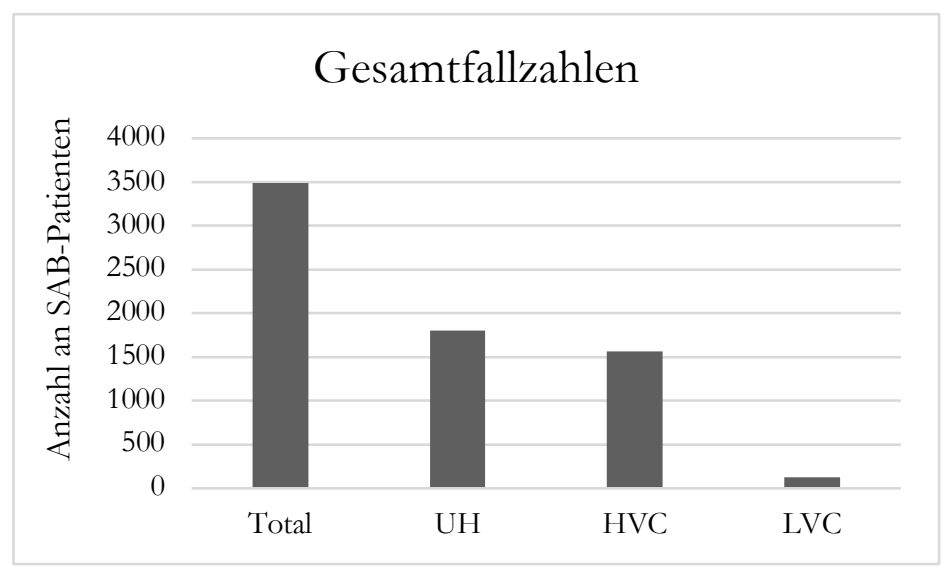

Abbildung 3: Gesamtbehandlungsvolumen. Absolute Anzahl an SAB-Patienten pro Jahr, aufgeteilt nach Art des Zentrums (UH, HVC, LVC und ingesamt (Total)). 


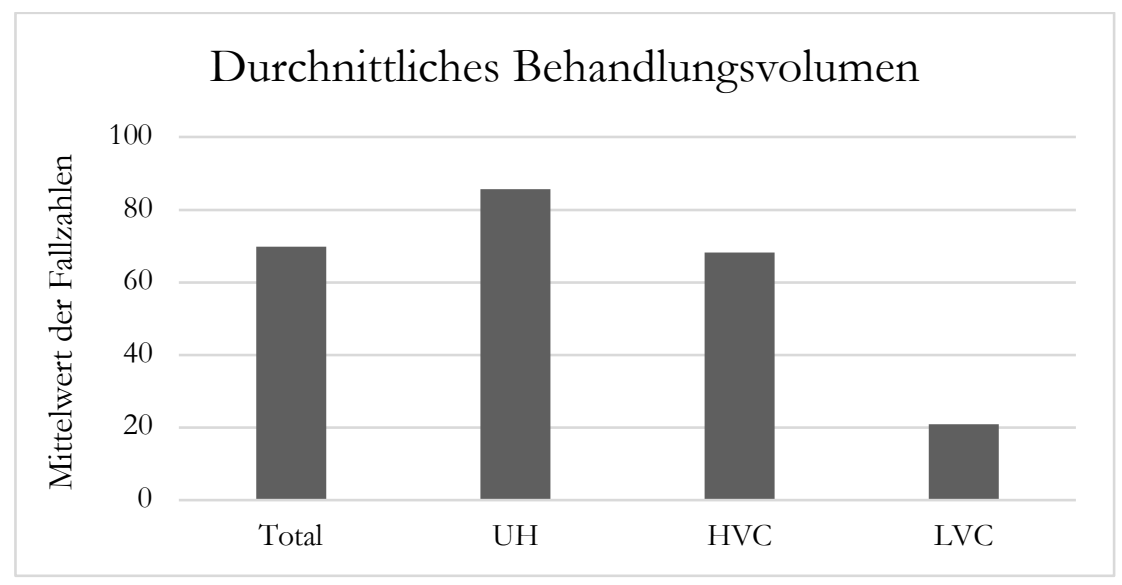

Abbildung 4: Durchschnittliches Behandlungsvolumen einer Klinik von SAB-Patienten pro Jahr. Aufgeteilt nach Art des Zentrums (UH, HVC, LVC und ingesamt (Total)). Dargestellt durch den Mittelwert der jeweils durch die einzelnen Zentren angegebenen Fallzahlen.

\subsection{Krankenhauseigenschaften}

Alle folgenden Zahlen zu den befragten Krankenhäusern beziehen sich, wenn nicht anderes beschrieben, auf die Einteilung nach dem behandelten Fallvolumen (Tabelle 9).

Die Antworten der Zentren bezüglich der Art der Leitung der ICU (rein neurochirurgisch, rein anästhesiologisch, gemischt, mit neurochirurgischer/neurologischer Mitarbeit) erwies sich als statistisch signifikant $(\mathrm{p}=0,012)$. Daraufhin wurden die Antworten in den Kategorien Mixed-ICU und Neuro-ICU zusammengefasst.

Die meisten Zentren gaben an, dass die ICU interdisziplinär geführt wird (Mixed-ICU: $\mathrm{n}=$ 42/50, 84\%; Neuro-ICU: $\mathrm{n}=8 / 50,16 \%$ ). Hier ergab sich kein statistisch signifikanter Unterschied $(\mathrm{p}=0,393)$.

Neuro-ICUs fanden sich am häufigsten bei UH ( $\mathrm{n}=5 / 8,62 \%)$, wobei die Neuro-ICUs bei allen UHs einen Anteil von $n=5 / 21$ (24\%) ausmachten. Bei den HVC machten die NeuroICUs einen Anteil von $n=2 / 8$ (25\%) von allen Neuro-ICUs aus, sowie einen Anteil von $n$ $=2 / 23(9 \%)$ von allen ICUs in der Kategorie HVC. Bei den LVC machten die Neuro-ICUS einen Anteil von $\mathrm{n}=1 / 8(12 \%)$, bezogen auf alle Neuro-ICUs aus, sowie einen Anteil von $\mathrm{n}=1 / 6(17 \%)$ von allen ICUs in der Kategorie der LVC. Aufgrund der stark unterschiedlichen Gruppengröße zwischen Mixed-ICUs ( $\mathrm{n}=42 / 50,84 \%$ ) und Neuro-ICUs ( $\mathrm{n}=8 / 50$, 16\%) wurde davon Abstand genommen statistische Vergleichsanalysen zwischen den beiden Gruppen durchzuführen, um einen Fehler zweiter Art zu vermeiden. 
Tabelle 9: Ergebnisse der Krankenhauseigenschaften

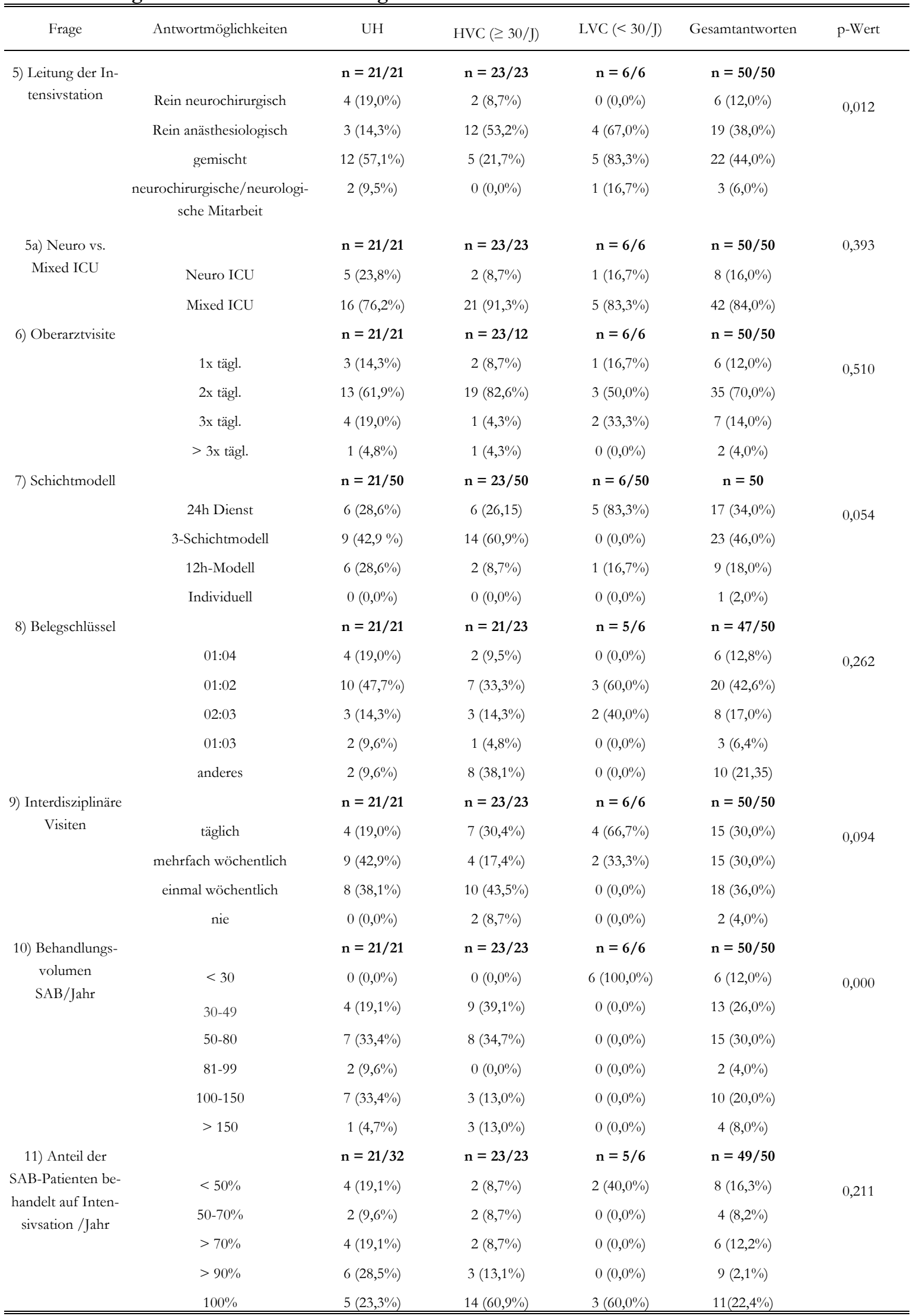




\subsection{Beatmungsmanagement}

Die meisten Zentren gaben keine definierten Werte an, die zur Indikationsstellung einer maschinellen Beatmung führen (Abbildung 6). Dieser Umstand verdeutlicht das unterschiedliche Verständnis, wann im Falle der SAB eine Beatmung durchführt werden sollte.

Bei Indikation zur maschinellen Beatmung aufgrund der Beurteilung der Vigilanz gaben $\mathrm{n}=$ 23/46 (50\%) Zentren an, dass der WFNS-Grad keine formale Indikation darstellt. Die andere Hälfte der Zentren, $\mathrm{n}=23 / 46$ (50\%) gab an, dass eine maschinelle Beatmung bei Grenzwerten von WFNS-Grad 3 und WFNS-Grad 4 regelmäßig und standartisiert durchgeführt wird $(p=0,411)$. Fast die Hälfte der Zentren gab an, dass der modizierte Fisher-Score eine Indikation zur maschinellen Beatmung darstellt $(n=21 / 47,45 \%)$. Für die andere Hälfte der Zentren stellte der modifizierte Fisher-Score keine Indikation dar $(n=26 / 47,55 \%)$. Hier wurde kein signifikanter Unterschied festgestellt $(p=0,102)$ (Tabelle 11).

58\% $(n=26 / 45)$ der Zentren verzichteten darauf, den Horowitz-Index zur Anpassung ihrer Beatmungseinstellung zu nutzen. Es ist anzunehmen, dass diese sich an anderen Parametern, wie beispielsweise am $\mathrm{paO}_{2}$, orientieren. Von den Zentren, die den Horowitz-Index nutzen, gaben die meisten Zentren einen Schwellenwert des Horowitz-Index von $<200 \mathrm{mmHg}$ an ( $n=16 / 26,62 \%$ ). Ein Drittel der Zentren intubierte die SAB-Patienten erst relativ spät, denn sie gaben einen Schwellenwert des Horowitz-Index von $<100 \mathrm{mmHg}$ an $(\mathrm{n}=10 / 26$, $38 \%$ ). Bei der Frage nach der Einstellung des $\mathrm{paO}_{2}$ und $\mathrm{paCO}_{2}$ zeigten sich folgende Ergebnisse. Eine Normokapnie von 35 bis $45 \mathrm{mmHg}(\mathrm{n}=42 / 45,93 \%)$ und Normoxie von 80 bis $100 \mathrm{mmHg}(\mathrm{n}=22 / 46,48 \%)$ wurde von den meisten Zentren angestrebt. Bei 35\% ( $\mathrm{n}=$ 16/46) der Zentren (25\% der UH; 45\% der HVC und 25\% der LVC) betrug der $\mathrm{paO}_{2}-$ Zielwert jedoch $>100 \mathrm{mmHg}$ (Tabelle 10). Es zeigten sich große Unterschiede bei den Zielwerten des $\mathrm{paO}_{2}(\mathrm{p}=0,244)$ und $\mathrm{paCO}_{2}(\mathrm{p}=0,497)$ (Tabelle 11 und Abbildung 5). Mehr als die Hälfte aller Krankenhäuser gab an, einen Optimumzielwert für den $\mathrm{paCO}_{2} \mathrm{zu}$ haben (n $=23 / 43,53 \%)$. Für den $\mathrm{paO}_{2}$ waren dies weniger $(\mathrm{n}=15 / 42,36 \%)($ Tabelle 11$)$.

\section{Tabelle 10: $\mathrm{paO}_{2}$-Zielwert (mmHg)}

\begin{tabular}{|c|c|c|c|c|c|c|}
\hline Frage & Antwortmöglichkeiten & $\mathrm{UH}$ & $\mathrm{HVC}(\geq 30 / \mathrm{J})$ & $\operatorname{LVC}(<30 / \mathrm{J})$ & Gesamtantworten & p-Wert \\
\hline \multirow{4}{*}{$\begin{array}{l}\text { 19) } \mathrm{paO} 2-\mathrm{Zielwert} \\
(\mathrm{mmHg})\end{array}$} & & $\mathrm{n}=20 / 21$ & $\mathrm{n}=22 / 23$ & $n=4 / 6$ & $n=46 / 50$ & \\
\hline & $80-100$ & $11(55,0 \%)$ & $10(45,5 \%)$ & $1(25,0 \%)$ & $22(47,8 \%)$ & 0,244 \\
\hline & $>100$ & $5(25,0 \%)$ & $10(45,5 \%)$ & $1(25,0 \%)$ & $16(34,8 \%)$ & \\
\hline & $\begin{array}{l}\text { Regulierung nach } \\
\qquad \mathrm{SaO} 2\end{array}$ & $4(20,0 \%)$ & $2(9,0 \%)$ & $2(50,0 \%)$ & $8(17,4 \%)$ & \\
\hline
\end{tabular}


Bezüglich der $\mathrm{paCO}_{2}$-Zielwerte herrschte Konsens zwischen den teilnehmenden Zentren zur Hypokapnie (Abbildung 5). 98\% der Zentren $(n=44 / 45)$ gaben an, dass eine Hypokapnie zu vermeiden ist. Desweiteren ergab die Umfrage, dass eine Hyperkapnie ebenfalls zu vermeiden ist, da nur 4\% der Zentren $(n=2 / 45)$ einen Zielwert $>45 \mathrm{mmHg}$ angaben. 93\% der Zentren $(\mathrm{n}=42 / 45)$ streben die Normokapnie an und gaben $\mathrm{paCO}_{2}$-Zielwerte von $35-45$ $\mathrm{mmHg}$ an (siehe Tabelle 11).

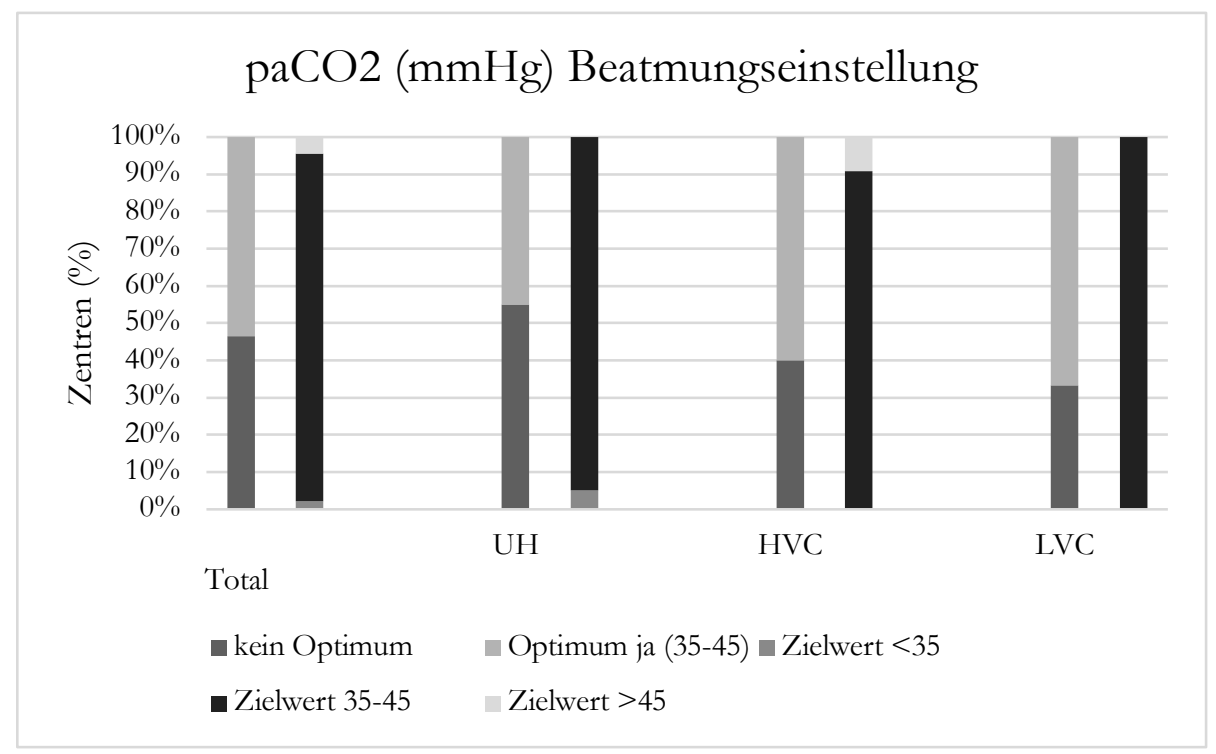

Abbildung 5: Indikationsstellung zur Beatmung. Höhe des angestrebten $\mathrm{paCO}_{2}(\mathrm{mmHg})(<35,35-45,>$ 45) und Existenz eines Optimum $\mathrm{paCO}_{2}$ auf den die Beatmungseinstellungen angepasst werden (ja, Normokapnie bei 35-45 mmHg/nein). Aufgeteilt nach Art des Zentrums (UH, HVC, LVC und ingesamt (Total)).

Zusammenfassend fanden sich große Unterschiede in den entscheidenden Punkten der Beatmungstherapie. Allerdings waren diese Unterschiede zwischen den verschiedenen Kategorien der Krankenhäuser und der unterschiedlichen Leitung der ICU statistisch nicht signifikant (Tabelle 11). 


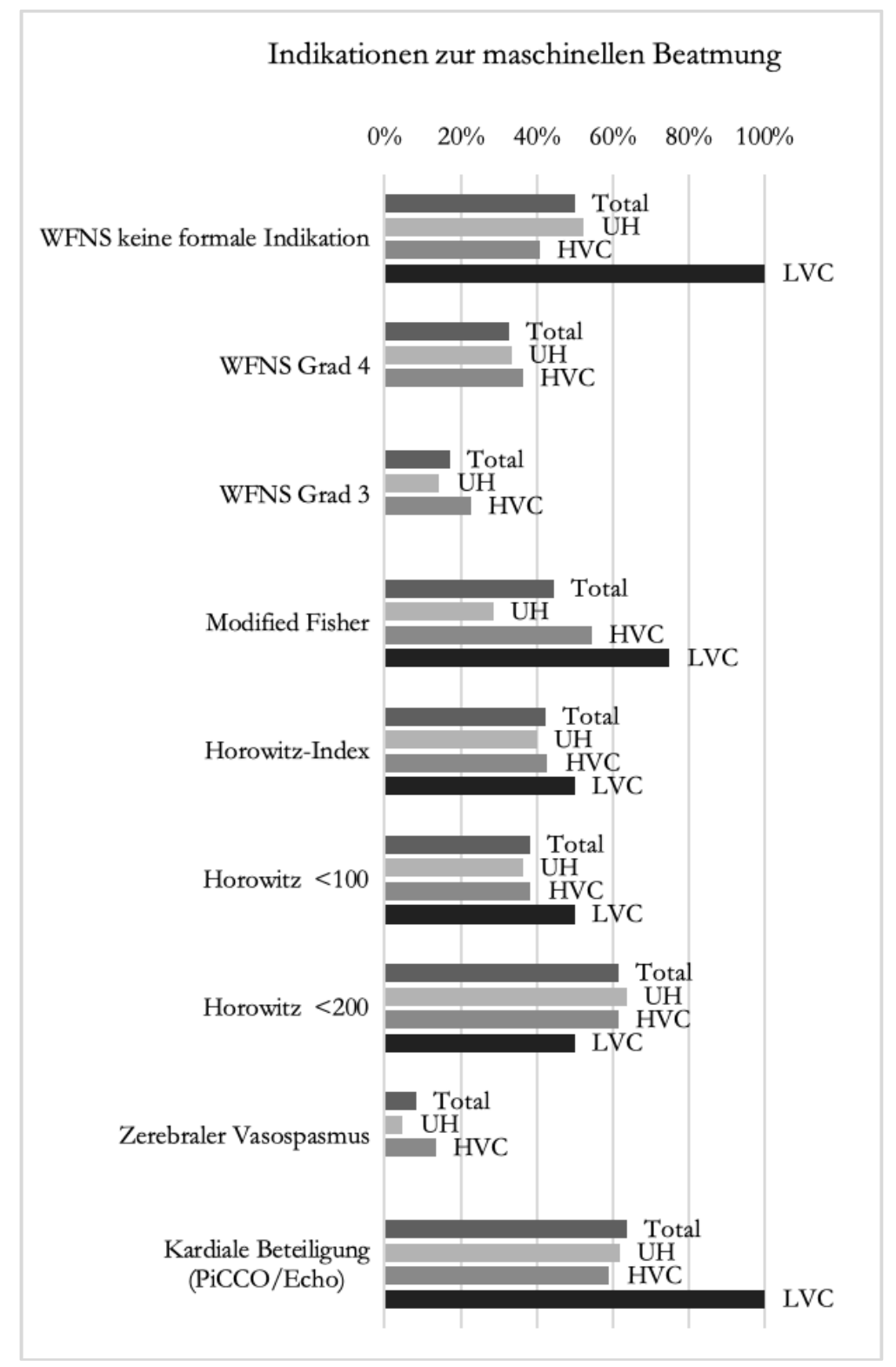

Abbildung 6: Parameter zur Indikationsstellung der mechanischen Ventilation. Aufgeteilt nach Art des Zentrums (UH, HVC, LVC und ingesamt (Total)). 
Tabelle 11: Ergebnisse zum Beatmungsmanagement

\begin{tabular}{|c|c|c|c|c|c|c|}
\hline Frage & Antwortmöglichkeiten & $\mathrm{UH}$ & HVC $(\geq 30 / J)$ & $\operatorname{LVC}(<30 / \mathrm{J})$ & Gesamtantworten & p-Wert \\
\hline \multirow{3}{*}{$\begin{array}{l}\text { 13) modified Fis- } \\
\text { her: Indikation zur } \\
\text { masch. Beatmung }\end{array}$} & & $n=21 / 21$ & $n=22 / 23$ & $n=4 / 6$ & $\mathrm{n}=47 / 50$ & \multirow{3}{*}{0,102} \\
\hline & ja & $6(28,6 \%)$ & $12(54,5 \%)$ & $3(75,0 \%)$ & $21(44,7 \%)$ & \\
\hline & nein & $15(71,4 \%)$ & $10(45,5 \%)$ & $1(25,0 \%)$ & $26(55,3 \%)$ & \\
\hline \multirow{4}{*}{$\begin{array}{l}\text { 14) WFNS als Indi- } \\
\text { kation zur maschi- } \\
\text { nellen Beatmung }\end{array}$} & & $n=21 / 21$ & $n=22 / 23$ & $n=3 / 6$ & $n=46 / 50$ & \multirow{4}{*}{0,411} \\
\hline & Grad 3 & $3(14,2 \%)$ & $5(22,7 \%)$ & $0(0,0 \%)$ & $8(17,4 \%)$ & \\
\hline & Grad 4 & $7(33,3 \%)$ & $8(36,4 \%)$ & $0(0,0 \%)$ & $15(32,6 \%)$ & \\
\hline & keine Indikation & $11(52,4 \% 9$ & $9(40,9 \%)$ & $3(100,0 \%)$ & $23(50,0 \%)$ & \\
\hline \multirow{3}{*}{$\begin{array}{l}\text { 15) Horowitz-In- } \\
\text { dex: Indikation zur } \\
\text { masch. Beatmung }\end{array}$} & & $\mathrm{n}=20 / 21$ & $\mathrm{n}=21 / 23$ & $n=4 / 6$ & $\mathrm{n}=45 / 50$ & \multirow{3}{*}{0,931} \\
\hline & ja & $8(40,0 \%)$ & $9(42,9 \%)$ & $2(50,0 \%)$ & $19(42,2 \%)$ & \\
\hline & nein & $12(60,0 \%)$ & $12(57,1 \%)$ & $2(50,0 \%)$ & $26(57,8 \%)$ & \\
\hline \multirow{3}{*}{$\begin{array}{l}\text { 16) Horowitz-In- } \\
\text { dex: Schwellenwert } \\
\text { für invasive Beat- } \\
\text { mung }\end{array}$} & & $n=11 / 21$ & $n=13 / 23$ & $n=2 / 6$ & $n=26 / 50$ & \multirow{3}{*}{0,936} \\
\hline & $<200$ & $7(63,6 \%)$ & $8(61,5 \%)$ & $1(50,0 \%)$ & $16(61,5 \%)$ & \\
\hline & $<100$ & $4(36,4 \%)$ & $5(38,5 \%)$ & $1(50,0 \%)$ & $10(38,5 \%)$ & \\
\hline \multirow{3}{*}{$\begin{array}{l}\text { 17) Zerebraler Va- } \\
\text { sospasmus als Indi- } \\
\text { kation zur Beat- } \\
\text { mung }\end{array}$} & & $n=21 / 21$ & $\mathrm{n}=22 / 23$ & $n=4 / 6$ & $\mathrm{n}=47 / 50$ & \multirow{3}{*}{0,474} \\
\hline & ja & $1(4,7 \%)$ & $3(13,6 \%)$ & $0(0,0 \%)$ & $4(8,5 \%)$ & \\
\hline & nein & $20(95,3 \%)$ & $19(86,4 \%)$ & $4(100,0 \%)$ & $43(91,5 \%)$ & \\
\hline 18) Schwere kardi- & & $n=21 / 21$ & $\mathrm{n}=22 / 23$ & $n=4 / 6$ & $\mathrm{n}=47 / 50$ & \multirow{4}{*}{0,307} \\
\hline $\begin{array}{l}\text { ale Mitbeteiligung } \\
\text { als Indikation zur }\end{array}$ & ja & $1(4,8 \%)$ & $3(13,6 \%)$ & $0(0,0 \%)$ & $4(8,5 \%)$ & \\
\hline \multirow{2}{*}{$\begin{array}{l}\text { maschinellen Beat- } \\
\text { mung und Anal- } \\
\text { gosedierung }\end{array}$} & nein & $8(38,1 \%)$ & $9(40,9 \%)$ & $0(0,0 \%)$ & $17(36,2 \%)$ & \\
\hline & $\begin{array}{l}\text { Abh. vom Schwere- } \\
\text { grad der kardialen Mit- } \\
\text { beteiligung } \\
\text { (PiCCO/Echo) }\end{array}$ & $12(57,1 \%)$ & $10(45,5 \%)$ & $4(100,0 \%)$ & $26(55,3 \%)$ & \\
\hline \multirow{4}{*}{$\begin{array}{l}\text { 20) } \mathrm{paCO} 2-\mathrm{Ziel}- \\
\text { wert }(\mathrm{mmHg})\end{array}$} & & $\mathrm{n}=20 / 21$ & $\mathrm{n}=22 / 23$ & $\mathrm{n}=3 / 6$ & $\mathrm{n}=45 / 50$ & \multirow{4}{*}{0,497} \\
\hline & $<35$ & $1(5,0 \%)$ & $0(0,0 \%)$ & $0(0,0 \%)$ & $1(2,2 \%)$ & \\
\hline & $35-45$ & $19(95,0 \%)$ & $20(91,0 \%)$ & $3(100,05)$ & $42(93,3 \%)$ & \\
\hline & $>45$ & $0(0,0 \%)$ & $2(9,0 \%)$ & $0(0,0 \%)$ & $2(4,4 \%)$ & \\
\hline 23) Optimum & & $\mathrm{n}=20 / 21$ & $n=19 / 23$ & $n=3 / 6$ & $\mathrm{n}=42 / 50$ & \\
\hline $\begin{array}{l}\text { paO2 zur Anpas- } \\
\text { sung der Beat- }\end{array}$ & $\mathrm{Ja}$ & $8(40,0 \%)$ & $7(36,8 \%)$ & $0(0,0 \%)$ & $15(35,7 \%$ & 0,399 \\
\hline mungseinstellungen & Nein & $12(60,0 \%)$ & $12(63,2 \%)$ & $3(100,0 \%)$ & $27(64,3)$ & \\
\hline 25) Optimum & & $\mathrm{n}=20 / 21$ & $n=20 / 23$ & $n=3 / 6$ & $n=43 / 50$ & \\
\hline $\begin{array}{l}\text { paCO2 zur Anpas- } \\
\text { sung der Beat- }\end{array}$ & $\mathrm{Ja}(35-45 \mathrm{mmHg})$ & $9(44,9 \%)$ & $12(60,0 \%)$ & $2(66,7 \%)$ & $23(53,5 \%)$ & 0,568 \\
\hline mungseinstellungen & Nein & $11(55,1 \%)$ & $8(40,0 \%)$ & $1(33,3 \%)$ & $20(46,5 \%)$ & \\
\hline \multirow{4}{*}{$\begin{array}{l}\text { 27) Standard Beat- } \\
\text { mungseinstellung }\end{array}$} & & $\mathrm{n}=21 / 21$ & $\mathrm{n}=22 / 23$ & $\mathrm{n}=3 / 6$ & $n=46 / 50$ & \multirow{4}{*}{0,129} \\
\hline & BIPAP & $18(85,7 \%)$ & $20(90,9 \%)$ & $2(66,7 \%)$ & $40(87,0 \%)$ & \\
\hline & Druckkontrolliert & $3(14,3 \%)$ & $0(0,0 \%)$ & $1(33,3 \%)$ & $4(8,7 \%)$ & \\
\hline & Volumenkontrolliert & $0(0,0 \%)$ & $2(9,1 \%)$ & $0(0,0 \%)$ & $2(4,3 \%)$ & \\
\hline
\end{tabular}




\subsection{Sedierungsmanagement}

Es machten n $=46 / 50(92 \%)$ der Zentren eine Angabe zum Anteil der intubierten und analgosedierten Patienten auf Intensivstation. Von diesen unterzogen $n=20 / 46$ (43\%) über die Hälfte ihrer SAB-Patienten einer Sedierung und maschineller Beatmung (Tabelle 12).

Tabelle 12: Darstellung der intubierten und analgosedierte Patienten auf der Intensivstation

\begin{tabular}{|c|c|c|c|c|c|c|}
\hline Frage & Antwortmöglichkeiten & UH & $\operatorname{HVC}(\geq 30 / \mathrm{J})$ & $\operatorname{LVC}(<30 / \mathrm{J})$ & Gesamtantworten & p-Wert \\
\hline \multirow{6}{*}{$\begin{array}{l}\text { 12) SAB-Patienten } \\
\text { intubiert/beatmet } \\
\text { und analgosediert }\end{array}$} & & $\mathrm{n}=21 / 21$ & $\mathrm{n}=21 / 23$ & $n=4 / 6$ & $n=46 / 50$ & \\
\hline & $<30 \%$ & $4(19,1 \%)$ & $5(23,8 \%)$ & $0(0,0 \%)$ & $9(19,6 \%)$ & 0,238 \\
\hline & $<50 \%$ & $7(33,3 \%)$ & $8(38,1 \%)$ & $2(50,0 \%)$ & $17(37,0 \%)$ & \\
\hline & $50-60 \%$ & $8(38,1 \%)$ & $6(28,6 \%)$ & $0(0,0 \%)$ & $14(30,45)$ & \\
\hline & $70-80 \%$ & $2(9,5 \%)$ & $0(0,0 \%)$ & $1(25,0 \%)$ & $3(6,5 \%)$ & \\
\hline & $>80 \%$ & $0(0,0 \%)$ & $2(9,5 \%)$ & $1(25,0 \%)$ & $3(6,5 \%)$ & \\
\hline
\end{tabular}

\subsubsection{Einleitung der Sedierung}

Es gaben insgesamt $n=44 / 50$ (88\%) der Zentren Informationen zu den Medikamenten an, die für die Induktion der Sedierung verwendet werden. Propofol wurde von der Mehrheit der Zentren eingesetzt ( $n=41 / 44,93 \%)$. Wie in Abbildung 7 dargestellt, zeigten sich große Differenzen zwischen UH, HVC und LVC. Propofol wurde unterschiedlich mit Opioiden, Benzodiazepinen, Ketamin und weiteren Muskelrelaxantien kombiniert (siehe Tabelle 13).

Beim Einsatz von Benzodiazepinen zeigte sich ein signifikanter Unterschied zwischen den Zentren. Benzodiazepine wurden signifikant häufiger von LVC eingesetzt $(p=0,044)$. Nur $\mathrm{n}=6 / 21(29 \%)$ der UH und $\mathrm{n}=6 / 20(30 \%)$ der HVC setzten Benzodiazepine ein (siehe Tabelle 13).

Tabelle 13: Medikamente zur Einleitung der Sedierung

\begin{tabular}{|c|c|c|c|c|c|c|}
\hline Frage & Antwortmöglichkeiten & $\mathrm{UH}$ & HVC $(\geq 30 / \mathrm{J})$ & $\mathrm{LVC}(<30 / \mathrm{J})$ & Gesamtantworten & p-Wert \\
\hline \multirow{7}{*}{$\begin{array}{l}\text { 22) Medikamente } \\
\text { der Wahl zur Einlei- } \\
\text { tung der Sedierung }\end{array}$} & & $\mathrm{n}=21 / 21$ & $\mathrm{n}=20 / 23$ & $n=3 / 6$ & $\mathrm{n}=44 / 50$ & \\
\hline & Ketamin & $0(0,0 \%)$ & $3(15,0 \%)$ & $1(33,3 \%)$ & $4(9,1 \%)$ & 0,079 \\
\hline & Benzodiazepin & $6(28,6 \%)$ & $6(30,0 \%)$ & $3(100,0 \%)$ & $15(34,1 \%)$ & 0,044 \\
\hline & Propofol & $20(95,2 \%)$ & $19(95,0 \%)$ & $2(66,7 \%)$ & $41(93,2 \%)$ & 0,168 \\
\hline & Kombination mit Opiat & $17(81,0 \%)$ & $19(95,0 \%)$ & $2(66,7 \%)$ & $38(86,4 \%)$ & 0,249 \\
\hline & Kombination mit Muskelrelaxans & $6(28,6 \%)$ & $5(25,0 \%)$ & $0(0,0 \%)$ & $11(25,0 \%)$ & 0,565 \\
\hline & anderes & $1(4,8 \%)$ & $0(0,0 \%)$ & $0(0,0 \%)$ & $1(2,3 \%)$ & 0,571 \\
\hline
\end{tabular}




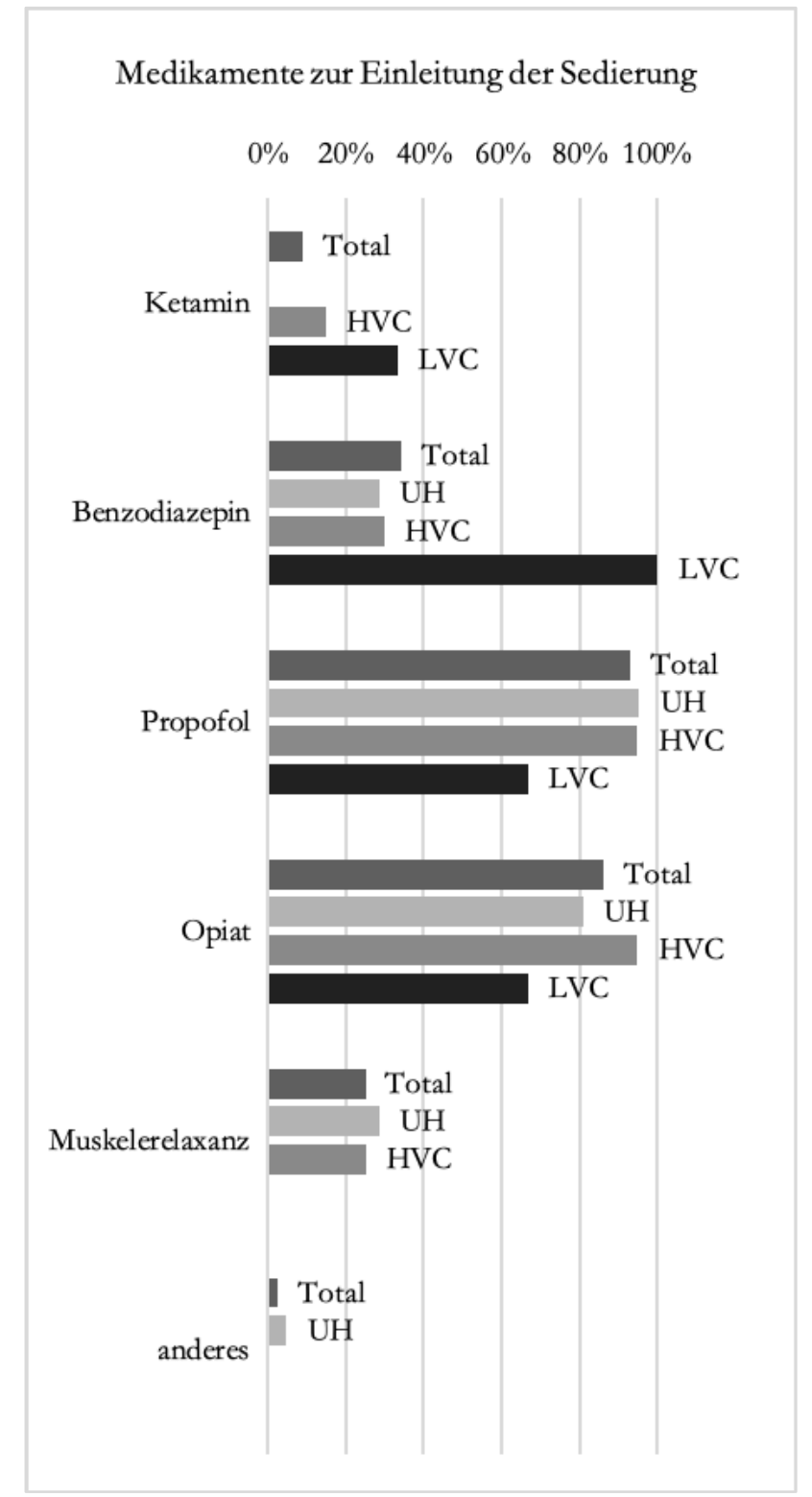

Abbildung 7: Medikamente zur Einleitung der Sedierung in den jeweiligen Zentren. Die meisten Zentren verwenden eine Kombination aus Propofol und Opioiden. Aufgeteilt nach Art des Zentrums (UH, HVC, LVC und ingesamt (Total)). 


\subsubsection{Aufrechterhaltung und Vertiefung der Sedierung}

Es berichteten $\mathrm{n}=38 / 50(76 \%)$ der Kliniken über eingesetzte Sedativa zur Aufrechterhaltung der Sedierung. Wie in Tabelle 14 und Abbildung 8 dargestellt, war eine große Variabilität zwischen und innerhalb der einzelnen Gruppen (UH, HVC und LVC) zu beobachten. Bemerkenswert war, dass nur ein einziges $\mathrm{UH}(\mathrm{n}=1 / 38,3 \%, \mathrm{p}=0,63)$ den Einsatz von volatilen Sedativa (hier Sevofluran) zur Aufrechterhaltung der Sedierung angab.

Ingesamt benutzten $n=9 / 38(24 \%)$ aller Zentren Ketamin zur Aufrechterhaltung der Sedierung. Davon waren es $\mathrm{n}=8 / 20(40 \%)$ der UH und nur $\mathrm{n}=1 / 17(6 \%)$ der HVC und keine LVC, welche Ketamin verwendeten. Die Anwendung von Ketamin der verschiedenen Zentren war signifikant auffällig $(p=0,044)$.

Insgesamt wurden am häufigsten Propofol $(n=28 / 38,74 \%)$ und Sufentanil $(n=23 / 38$, 61\%) zur Aufrechterhaltung der Sedierung eingesetzt. Nur bei den LVC unterschied sich dies. Von diesen wurde nur Propofol und Midazolam angegeben.

Tabelle 14: Medikamente zur Aufrechterhaltung der Sedierung

\begin{tabular}{|c|c|c|c|c|c|c|}
\hline Frage & Antwortmöglichkeiten & $\mathrm{UH}$ & $\mathrm{HVC}(\geq 30 / \mathrm{J})$ & $\operatorname{LVC}(<30 / \mathrm{J})$ & Gesamtantworten & $\mathrm{p}$-Wert \\
\hline \multirow{10}{*}{$\begin{array}{l}\text { 27) Medikamente zur Auf- } \\
\text { rechterhaltung der Sedierung }\end{array}$} & & $\mathrm{n}=20 / 21$ & $n=17 / 23$ & $n=1 / 6$ & $\mathrm{n}=38 / 50$ & \\
\hline & Propofol & $16(80,0 \%)$ & $11(64,7 \%)$ & $1(100,0 \%)$ & $28(73,7 \%)$ & 0,478 \\
\hline & Remifentanil & $3(15,0 \%)$ & $1(5,9 \%)$ & $0(0,0 \%)$ & $4(10,5 \%)$ & 0,628 \\
\hline & Midazolam & $10(50,0 \%)$ & $11(64,7 \%)$ & $1(100,0 \%)$ & $22(579 \%)$ & 0,458 \\
\hline & Sufentanil & $13(65,0 \%)$ & $10(58,8 \%)$ & $0(0,0 \%)$ & $23(60,5 \%)$ & 0,423 \\
\hline & Clonidin & $3(15,0 \%)$ & $0(0,0 \%)$ & $0(0,0 \%)$ & $3(7,9 \%)$ & 0,231 \\
\hline & Ketamin & $8(40,0 \%)$ & $1(5,9 \%)$ & $0(0,0 \%)$ & $9(23,7 \%)$ & 0,044 \\
\hline & Fentanyl & $2(10,0 \%)$ & $4(23,5 \%)$ & $0(0,0 \%)$ & $6(23,7 \%)$ & 0,482 \\
\hline & Methohexital & $0(0,0 \%)$ & $1(5,9 \%)$ & $0(0,0 \%)$ & $1(2,6 \%)$ & 0,530 \\
\hline & Sevofluran/Isofluran & $1(5,0 \%)$ & $0(0,0 \%)$ & $0(0,0 \%)$ & $1(2,6 \%)$ & 0,630 \\
\hline \multirow{3}{*}{$\begin{array}{l}\text { 24) Einsatz von Benzodiaze- } \\
\text { pin zur Aufrechterhaltung der } \\
\text { Sedierung }\end{array}$} & & $n=19 / 21$ & $n=19 / 23$ & $\mathrm{n}=3 / 6$ & $n=41 / 50$ & \\
\hline & Midazolam & $15(78,9 \%)$ & $13(68,4 \%)$ & $3(100,0 \%)$ & $31(75,5 \%)$ & 0,446 \\
\hline & nein & $4(21,1 \%)$ & $6(31,6 \%)$ & $0(0,0 \%)$ & $10(24,4 \%)$ & \\
\hline \multirow{5}{*}{$\begin{array}{l}\text { 28) Einsatz von einem Opioid } \\
\text { zur Aufrechterhaltung der Se- } \\
\text { dierung }\end{array}$} & & $\mathrm{n}=20 / 21$ & $n=20 / 23$ & $\mathrm{n}=3 / 6$ & $\mathrm{n}=43 / 50$ & \\
\hline & Remifentanil & $3(13,3 \%)$ & $2(10,0 \%)$ & $0(0,0 \%)$ & $5(11,6 \%)$ & 0,716 \\
\hline & Fentanyl & $2(8,8 \%)$ & $6(30,0 \%)$ & $0(0,0 \%)$ & $8(18,6 \%)$ & 0,185 \\
\hline & Sufentanil & $18(90,0 \%)$ & $16(80,0 \%)$ & $3(100,0 \%)$ & $37(86,0 \%)$ & 0,508 \\
\hline & nein & $2(8,8 \%)$ & $0(0,0 \%)$ & $0(0,0 \%)$ & $2(4,7 \%)$ & 0,299 \\
\hline
\end{tabular}




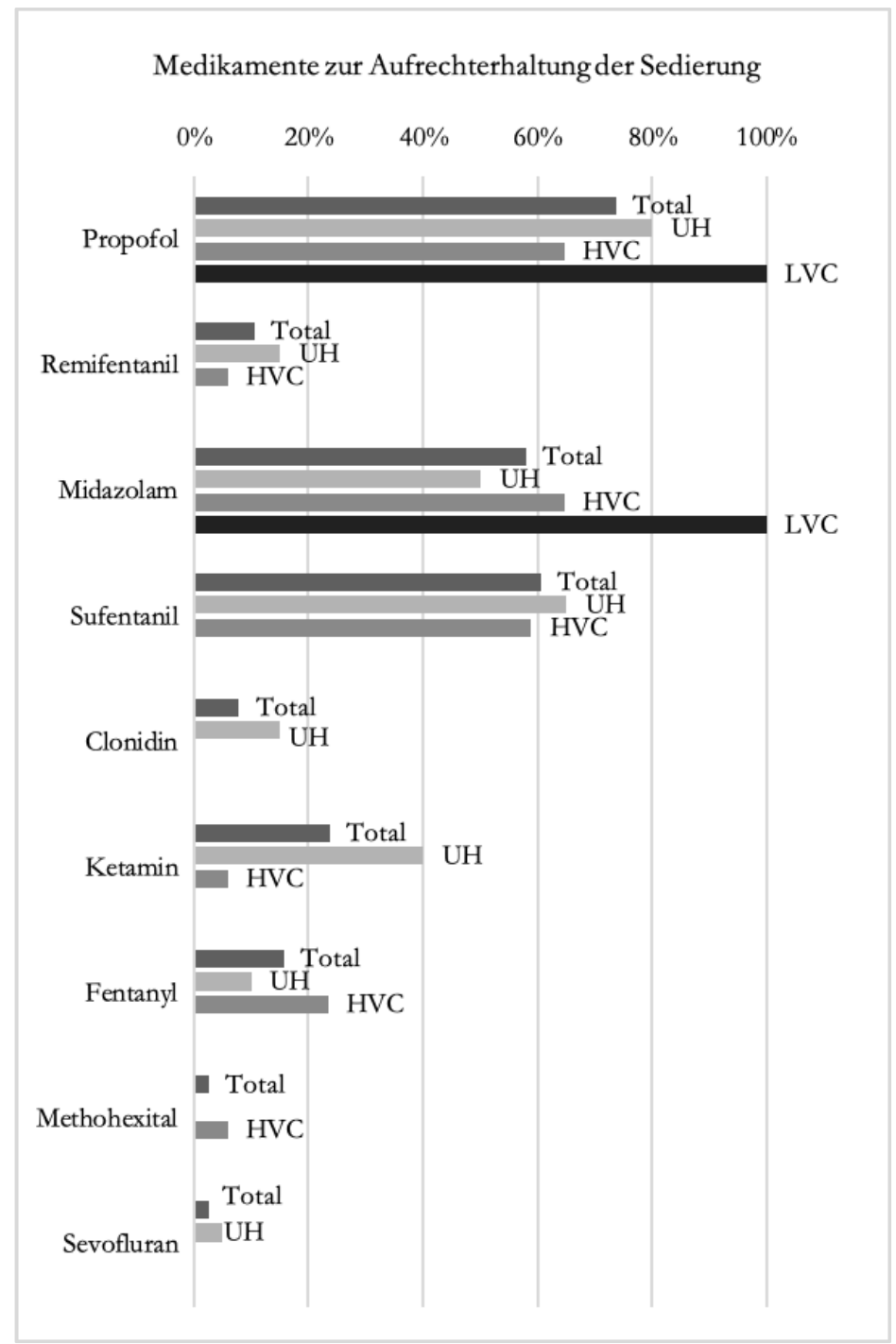

Abbildung 8: Medikamente zur Aufrechterhaltung der Sedierung. Eingeteilt nach Art des Zentrums (UH, HVC, LVC und ingesamt (Total)). Die meisten Zentren verwendeten eine Kombination aus Propofol, Midazolam und Sufentanil, um die Sedierung bei SAB-Patienten aufrechtzuerhalten. Nur ein UH ( $\mathrm{n}=1 / 21,5 \%$ ) verwendete inhalative Sedativa.

Ebenso gab es keine Übereinstimmung beim Einsatz von Medikamenten zur Vertiefung der Sedierung. Die Frage wurde von $n=43 / 50$ (86\%) der Zentren beantwortet (Tabelle 15). Eine Vielzahl verschiedener Medikamente wurde angegeben. Es zeigte sich aus den Daten (Abbildung 9) ein Trend, dass die meisten Kliniken ( $n=29 / 43$, 67\%) Ketamin einsetzten und UH ( $\mathrm{n}=15 / 20,75 \%), \mathrm{HVC}(\mathrm{n}=12 / 20,60 \%)$ jeweils ebenso am häufigsten Ketamin verwendeten. Die LVC verwendeten Clonidin ( $\mathrm{n}=3 / 3,100 \%$ ), häufiger als Ketamin ( $\mathrm{n}=$ 2/3, 67\%) zur Vertiefung der Sedierung. Dieser Unterschied zeigte sich als statistisch signifikant $(p=0,009)$. Zudem zeigte sich eine Signifikanz $(p=0,039)$ beim nur von UH verwendetem zusätzlichem Opioid ( $\mathrm{n}=5 / 20,25 \%)$. Als ,weitere“ Medikamente ( $\mathrm{n}=5 / 43,12 \%)$ wurde als Freitextantwort die Verwendung von Sufentanil, Metohexital, Midazolam, Thiopental und Pentobarbital angegeben. 
Tabelle 15: Medikamente zur Vertiefung der Sedierung

\begin{tabular}{|c|c|c|c|c|c|c|}
\hline Frage & Antwortmöglichkeiten & $\mathrm{UH}$ & $\operatorname{HVC}(\geq 30 / J)$ & $\operatorname{LVC}(<30 / \mathrm{J})$ & Gesamtantworten & $\mathrm{p}$-Wert \\
\hline \multirow{8}{*}{$\begin{array}{l}\text { 38) Medikament der ersten } \\
\text { Wahl um die Sedierung zu } \\
\text { vertiefen }\end{array}$} & & $\mathrm{n}=20 / 21$ & $\mathrm{n}=20 / 23$ & $\mathrm{n}=3 / 6$ & $n=43 / 50$ & \\
\hline & Gammahydroxybutyrat & $2(10,1 \%)$ & $1(5,0 \%)$ & $0(0,0 \%)$ & $3(7,0 \%)$ & 0,731 \\
\hline & Clonidin & $4(20,0 \%)$ & $4(20,0 \%)$ & $3(100,0 \%)$ & $11(25,6 \%)$ & 0,009 \\
\hline & anderes Benzodiazepin & $5(24,9 \%)$ & $2(10,0 \%)$ & $1(33,3 \%)$ & $8(18,6 \%)$ & 0,378 \\
\hline & Ketamin & $15(75,0 \%)$ & $12(60,0 \%)$ & $2(66,7 \%)$ & $29(67,4 \%)$ & 0,599 \\
\hline & Propofol & $11(55,0 \%)$ & $8(40,0 \%)$ & $2(66,7 \%)$ & $21(48,8 \%)$ & 0,519 \\
\hline & anderes Opioid & $5(24,9 \%)$ & $0(0,0 \%)$ & $0(0,0 \%)$ & $5(11,6 \%)$ & 0,039 \\
\hline & weitere & $1(4,9 \%)$ & $4(20,0 \%)$ & $0(0,0 \%)$ & $5(11,6 \%)$ & 0,247 \\
\hline \multirow{8}{*}{$\begin{array}{l}\text { 40) Weiteres Medikament } \\
\text { um die Sedierung zu vertie- } \\
\text { fen }\end{array}$} & & $\mathrm{n}=20 / 21$ & $n=20 / 23$ & $\mathrm{n}=3 / 6$ & $n=43 / 50$ & \\
\hline & Gammahydroxybutyrat & $2(10,1 \%)$ & $2(10,0 \%)$ & $0(0,0 \%)$ & $4(9,3 \%)$ & 0,848 \\
\hline & Clonidin & $6(30,1 \%)$ & $8(40,0 \%)$ & $2(66,7 \%)$ & $16(37,2 \%)$ & 0,444 \\
\hline & anderes Benzodiazepin & $7(35,0 \%)$ & $5(25,0 \%)$ & $1(33,3 \%)$ & $13(30,2 \%)$ & 0,783 \\
\hline & Ketamin & $12(60,2 \%)$ & $15(75,0 \%)$ & $2(66,7 \%)$ & $29(67,4 \%)$ & 0,599 \\
\hline & Propofol & $11(55,0 \%)$ & $11(55,0 \%)$ & $2(66,7 \%)$ & $24(55,8 \%)$ & 0,926 \\
\hline & anderes Opioid & $5(24,9 \%)$ & $6(30,0 \%)$ & $0(0,0 \%)$ & $11(25,6 \%)$ & 0,538 \\
\hline & anderes & $4(20,0 \%)$ & $1(5,0 \%)$ & $0(0,0 \%)$ & $5(11,6 \%)$ & 0,271 \\
\hline
\end{tabular}




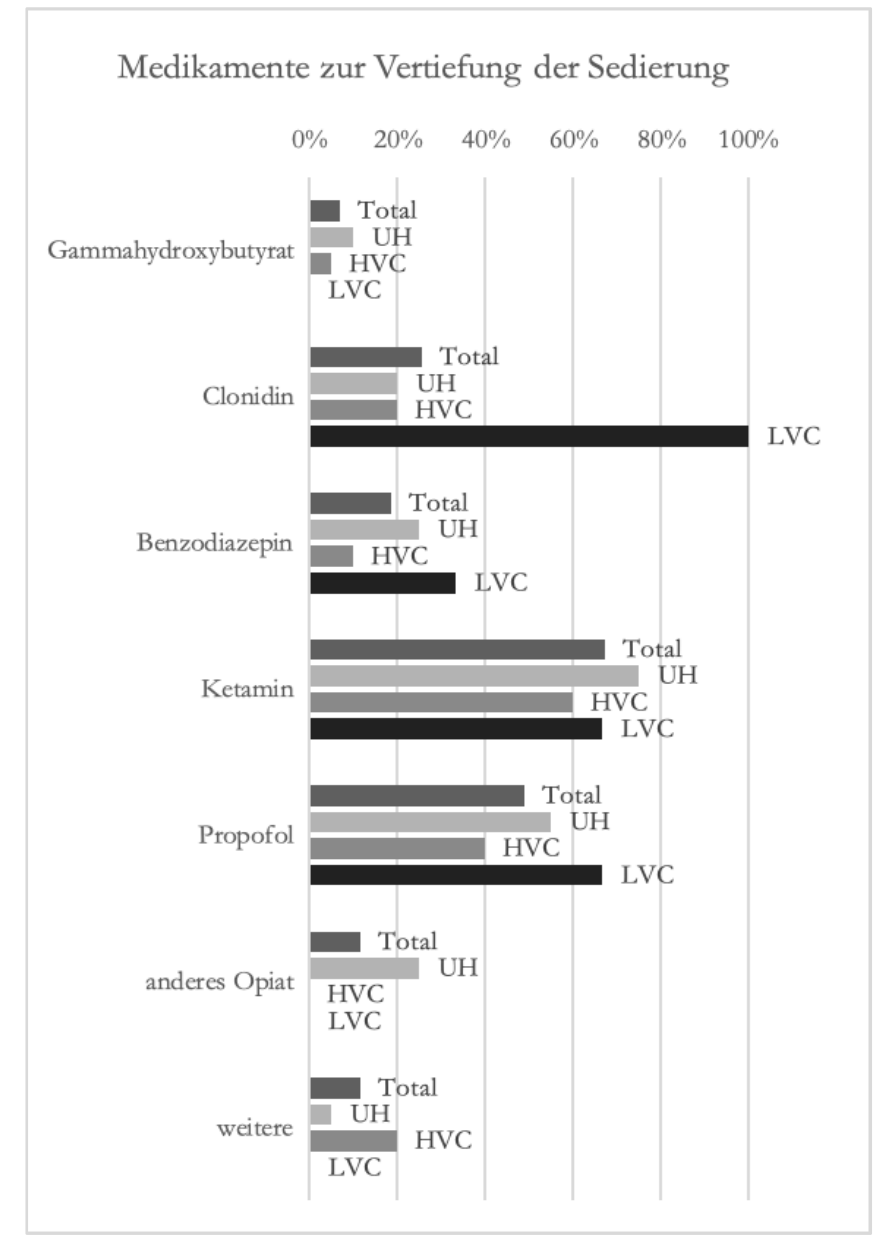

Abbildung 9: Medikamente zur Vertiefung der Sedierung. Aufgeteilt nach Art des Zentrums (UH, HVC, LVC und ingesamt (Total)). Ketamin war das am häufigsten verwendete Medikament von UH und HVC.

UH und HVC berichteten von einem höherem Gebrauch von Barbituraten als die LVC, um die Sedierung zu vertiefen. Dieser Unterschied zeigte sich als nicht statistisch signifikant (p $=0,297)$ (Tabelle 16). Die Zentren gaben zu einem Großteil an $(n=29 / 42,69 \%)$, dass die Gabe von Barbituraten kurzzeitig erfolgt.

Tabelle 16: Vertiefung der Sedierung: Gabe eines Barbiturats und Dauer der Administration

\begin{tabular}{|c|c|c|c|c|c|c|}
\hline Frage & Antwortmöglichkeiten & $\mathrm{UH}$ & $\operatorname{HVC}(\geq 30 / \mathrm{J})$ & $\operatorname{LVC}(<30 / \mathrm{J})$ & Gesamtantworten & p-Wert \\
\hline \multirow{3}{*}{$\begin{array}{l}\text { 47) Barbiturate um Sedie- } \\
\text { rung weiter zu vertiefen }\end{array}$} & & $\mathrm{n}=20 / 21$ & $n=20 / 23$ & $\mathrm{n}=3 / 6$ & $\mathrm{n}=43 / 50$ & \\
\hline & ja & $15(75,0 \%)$ & $12(60,0 \%)$ & $1(33,3 \%)$ & $28(65,1 \%)$ & 0,297 \\
\hline & nein & $5(25,0 \%)$ & $8(40,0 \%)$ & $2(66,7 \%)$ & $15(34,9 \%)$ & \\
\hline \multirow{4}{*}{$\begin{array}{l}\text { 48) Länge der Barbitu- } \\
\text { ratgabe }\end{array}$} & & $n=20 / 21$ & $n=19 / 23$ & $n=3 / 6$ & $\mathrm{n}=42 / 50$ & \\
\hline & kurzzeitig & $14(70,0 \%)$ & $14(73,7 \%)$ & $1(33,3 \%)$ & $29(69,0 \%)$ & 0,583 \\
\hline & längerfristig & $1(5,0 \%)$ & $1(5,3 \%)$ & $0(0,0 \%)$ & $2(4,8 \%)$ & \\
\hline & keine Anwendung & $5(25,0 \%)$ & $4(21,2 \%)$ & $2(66,7 \%)$ & $11(26,2)$ & \\
\hline
\end{tabular}


Die meisten Krankenhäuser $(\mathrm{n}=33 / 43,77 \%$ ) gaben als maximal Anzahl der Sedativa drei oder mehr Medikamente an, wobei keine statistische Signifikanz zwischen UH, HVC und LVC bestand $(\mathrm{p}=0,851)$ (Tabelle 17).

Tabelle 17: Maximale Anzahl an verwendeten Sedativa zur Vertiefung der Sedierung

\begin{tabular}{ccccccc}
\hline \hline Frage & Antwortmöglichkeiten & UH & HVC $(\geq 30 / \mathrm{J})$ & LVC $(<30 / \mathrm{J})$ & Gesamtantworten & p-Wert \\
\hline $\begin{array}{c}\text { 39) Maximale Anzahl an } \\
\text { verwendeten Sedativa }\end{array}$ & & $\mathbf{n}=\mathbf{2 0 / 2 1}$ & $\mathbf{n}=\mathbf{2 0 / 2 3}$ & $\mathbf{n}=\mathbf{3 / 6}$ & $\mathbf{n}=\mathbf{4 3 / 5 0}$ & \\
& 2 & $5(24,9 \%)$ & $4(20,0 \%)$ & $1(33,3 \%)$ & $10(23,3 \%)$ & 0,851 \\
& $\mathbf{2}$ & $15(75,0 \%)$ & $16(80,0 \%)$ & $2(66,7 \%)$ & $33(76,7 \%)$ & \\
\hline \hline
\end{tabular}

\subsubsection{Dosierung der Sedativa}

Eine weitere Beobachtung zeigte die erhebliche Variabilität in der Dosierung der Sedativa der unterschiedlichen Zentren, welches sich aus den eingesetzten Maximaldosierungen ablesen ließ (Tabelle 18). Die Krankenhäuser wurden nach der Maximumdosis für das Benzodiazepin Midazolam befragt. Die maximalen Dosierungen schwankten von $0,1 \mathrm{mg} / \mathrm{kgKG} / \mathrm{h}$ bis zu $0,75 \mathrm{mg} / \mathrm{kgKG} / \mathrm{h}$. Diese Unterschiede wurden auch bei der Auswertung der maximalen Dosierungen des Opioids Sufentanil ersichtlich. Die maximalen Dosierungen variierten von $0,3 \mu \mathrm{g} / \mathrm{kgKG} / \mathrm{h}$ bis $>2 \mu \mathrm{g} / \mathrm{kgKG} / \mathrm{h}$ (Abbildung 10).

Tabelle 18: Dosierung von Midazolam und Sufentanil

\begin{tabular}{|c|c|c|c|c|c|c|}
\hline Frage & Antwortmöglichkeiten & UH & HVC & $\operatorname{LVC}(<30 / \mathrm{J})$ & Gesamtantworten & p-Wert \\
\hline \multirow{5}{*}{$\begin{array}{l}\text { 29) Maximale Dosierung } \\
\text { des Benzodiazepins } \\
\text { (Midazolam) in } \\
\mathrm{mg} / \mathrm{kgKG} / \mathrm{h}\end{array}$} & & $\mathrm{n}=14 / 21$ & $\mathrm{n}=7 / 23$ & $\mathrm{n}=1 / 6$ & $\mathrm{n}=22 / 50$ & \multirow{5}{*}{0,517} \\
\hline & $\leq 0,25 \mathrm{mg} / \mathrm{kgKG} / \mathrm{h}$ & $5(35,7 \%)$ & $6(85,7 \%)$ & $1(100,0 \%)$ & $12(54,5 \%)$ & \\
\hline & $\leq 0,45 \mathrm{mg} / \mathrm{kgKG} \mathrm{h}$ & $3(21,4 \%)$ & $1(14,3 \%)$ & $0(0,0 \%)$ & $4(18,2 \%)$ & \\
\hline & $0,5-0,75 \mathrm{mg} / \mathrm{kgKG} / \mathrm{h}$ & $5(35,7 \%)$ & $0(0,0 \%)$ & $0(0,0 \%)$ & $5(22,7 \%)$ & \\
\hline & individuell, nach Wirkung & $1(7,1 \%)$ & $0(0,0 \%)$ & $0(0,0 \%)$ & $1(4,5 \%)$ & \\
\hline \multirow{6}{*}{$\begin{array}{l}\text { 30) Maximale Dosierung } \\
\text { des Opioids (Sufentanil) }\end{array}$} & & $\mathrm{n}=14 / 21$ & $\mathrm{n}=8 / 23$ & $\mathrm{n}=1 / 6$ & $n=23 / 50$ & \multirow{6}{*}{0,312} \\
\hline & $0,3-0,5 \mu \mathrm{g} / \mathrm{kgKG} / \mathrm{h}$ & $2(14,3 \%)$ & $4(50,0 \%)$ & $1(100,0 \%)$ & $7(30,4 \%)$ & \\
\hline & $0,6-0,9 \mu \mathrm{g} / \mathrm{kgKG} / \mathrm{h}$ & $5(35,7 \%)$ & $0(0,0 \%)$ & $0(0,0 \%)$ & $5(21,7 \%)$ & \\
\hline & $1-1,5 \mu \mathrm{g} / \mathrm{kgKG} / \mathrm{h}$ & $3(21,4 \%)$ & $3(37,5 \%)$ & $0(0,0 \%)$ & $6(26,1 \%)$ & \\
\hline & $1,6-2 \mu \mathrm{g} / \mathrm{kgKG} / \mathrm{h}$ & $2(14,3 \%)$ & $1(2,5 \%)$ & $0(0,0 \%)$ & $3(13,0 \%)$ & \\
\hline & individuell, nach Wirkung & $2(14,3 \%)$ & $0(0,0 \%)$ & $0(0,0 \%)$ & $2(8,7 \%)$ & \\
\hline
\end{tabular}




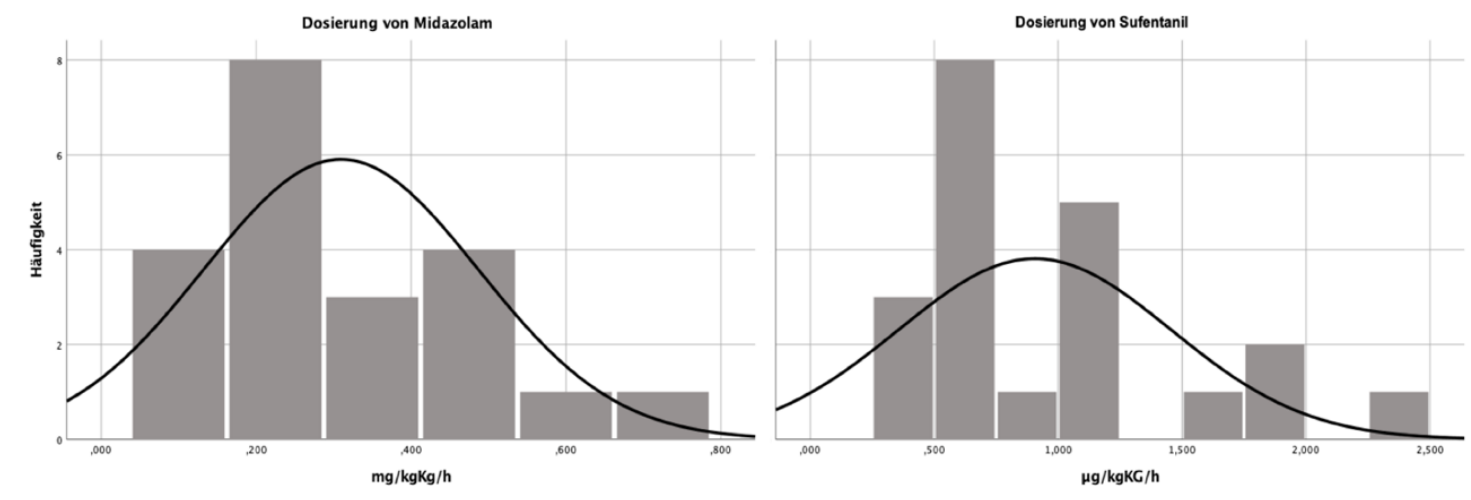

Abbildung 10: Variabilität der maximalen Dosierungen am Beispiel von Midazolam und Sufentanil. Die maximalen Dosierungen für Midazolam variierten von $0,1 \mathrm{mg} / \mathrm{kgKG} / \mathrm{h}$ bis $0,75 \mathrm{mg} / \mathrm{kgKG} / \mathrm{h}$ ). Die Domaximalen sierungen des Opioids Sufentanil variierten von $0,3 \mu \mathrm{g} / \mathrm{kgKG} / \mathrm{h}$ bishin zu $>2 \mu \mathrm{g} / \mathrm{kgKG} / \mathrm{h}$. Basierend auf den veröffentlichten Daten von Hernández-Durán et al. (2020).

Ketamin wurde besonders häufig zur Vertiefung der Sedierung verwendet ( $\mathrm{n}=29 / 43,67 \%$ ). Die Dosierungen variierten deutlich. Nicht nur gab es einen großen Unterschied zwischen den Antworten der Zentren, sondern auch in den jeweiligen Antworten innerhalb der drei Zentrentypen, wie den Angaben der Tabelle 19 deutlich zu entnehmen ist. Am häufigsten wurde die Dosierung von 2,5 bis $4 \mathrm{mg} / \mathrm{kgKG} / \mathrm{h}$ angegeben $(\mathrm{n}=12 / 27,44 \%)$. Die meisten Zentren verneinten ein zusätzliches kardiales Monitoring während der Ketaminapplikation ( $\mathrm{n}=29 / 38,76 \%$ ). Von den UH gaben $\mathrm{n}=5 / 20$ (25\%) und von den HVC $\mathrm{n}=4 / 17$ (24\%) allerdings an ein kardiales Monitoring durchzuführen $(\mathrm{p}=0,848)$.

Tabelle 19: Einsatz von Ketamin im Rahmen der Sedierung

\begin{tabular}{|c|c|c|c|c|c|c|}
\hline Frage & Antwortmöglichkeiten & $\mathrm{UH}$ & $(\geq$ & $(<30 / \mathrm{J})$ & esamtantwort & $\mathrm{p}$-Wert \\
\hline \multirow[t]{5}{*}{ 45) Dosierung von Ketamin } & & $n=14 / 21$ & $n=12 / 23$ & $n=1 / 6$ & $\mathrm{n}=27 / 50$ & \multirow{5}{*}{0,669} \\
\hline & $0,3-1 \mathrm{mg} / \mathrm{kgKG} / \mathrm{h}$ & $3(21,4 \%)$ & $3(25,0 \%)$ & $0(0,0 \%)$ & $6(22,2 \%)$ & \\
\hline & $1,25-2 \mathrm{mg} / \mathrm{kgKG} / \mathrm{h}$ & $3(21,4 \%)$ & $2(16,7 \%)$ & $0(0,0 \%)$ & $5(18,5 \%)$ & \\
\hline & 2,5-4 mg/kgKG/h & $7(50,0 \%)$ & $4(33,3 \%)$ & $1(100,0 \%)$ & $12(44,4 \%)$ & \\
\hline & $5-8 \mathrm{mg} / \mathrm{kgKG} / \mathrm{h}$ & $1(7,1 \%)$ & $3(25,0 \%)$ & $0(0,0 \%)$ & $4(14,8 \%)$ & \\
\hline \multirow{3}{*}{$\begin{array}{l}\text { 46) Weiteres kardiales Monitor- } \\
\text { ing während Ketaminapplikation }\end{array}$} & & $n=20 / 21$ & $n=17 / 23$ & $n=1 / 6$ & $\mathrm{n}=38 / 50$ & \multirow{3}{*}{0,848} \\
\hline & ja & $5(25,0 \%)$ & $4(23,5 \%)$ & $0(0,0 \%)$ & $9(23,7 \%)$ & \\
\hline & nein & $15(75,0 \%)$ & $13(76,5 \%)$ & $1(100,0 \%)$ & $29(76,3 \%)$ & \\
\hline
\end{tabular}

Wie in den vorangegangenen Kapiteln verdeutlicht wurde, kam Propofol sowohl zur Einleitung und Aufrechterhaltung, sowie zur Vertiefung der Sedierung zum Einsatz. Die übliche Dosierung (siehe Tabelle 20) wurde mehrheitlich zwischen 2 bis $4 \mathrm{mg} / \mathrm{kgKG} / \mathrm{h}(\mathrm{n}=23 / 31$, $74 \%$ ) angegeben. 
Insgesamt wurde über die verschiedenen Zentren hinweg eine sehr geringe Rate $(<1 \%)$ von Komplikationen im Rahmen der Propofolgabe wahrgenommen ( $n=29 / 43,67 \%)$. Wenn Komplikationen attributiert wurden, wurde von ähnliche Komplikationen berichtet. Hervorzuheben waren hier als häufigste Komplikationen der Hypotonus und das Propofol-Infusionssyndrom (PRIS), jeweils zu n = 14/33 (42\%). Als statistisch signifikant präsentierte sich das Auftreten einer Erhöhung der Kreatinkinasen (CK), welches von n = 2/15 (13\%) der HVC, keinem UH und einem LVC angegeben wurde $(p=0,002)$. Propofol wurde von $n=$ 25/39 (64\%) aller Zentren für maximal sieben Tage verwendet. Jeweils $n=7 / 39(18 \%)$ aller Zentren gaben eine Dauer von maximal drei, respektive mehr als sieben Tage an.

Tabelle 20: Einsatz von Propofol im Rahmen der Sedierung

\begin{tabular}{|c|c|c|c|c|c|c|}
\hline Frage & Antwortmöglichkeiten & $\mathrm{UH}$ & $\operatorname{HVC}(\geq 30 / \mathrm{J})$ & $\operatorname{LVC}(<30 / \mathrm{J})$ & Gesamtantwort & p-Wert \\
\hline \multirow{5}{*}{$\begin{array}{l}\text { 41) Bevorzugte Dosierung von } \\
\text { Propofol }\end{array}$} & & $\mathrm{n}=15 / 21$ & $\mathrm{n}=15 / 23$ & $\mathrm{n}=1 / 6$ & $\mathrm{n}=31 / 50$ & \\
\hline & $2-3 \mathrm{mg} / \mathrm{kgKG} / \mathrm{h}$ & $4(26,7 \%)$ & $4(26,7 \%)$ & $0(0,0 \%)$ & $8(25,8 \%)$ & 0,893 \\
\hline & 3,1-4 mg/KGkg/h & $6(40,0 \%)$ & $8(53,3 \%)$ & $1(100,0 \%)$ & $15(48,4 \%)$ & \\
\hline & $5-6,25 \mathrm{mg} / \mathrm{kgKG} / \mathrm{h}$ & $5(33,3 \%)$ & $1(6,7 \%)$ & $0(0,0 \%)$ & $6(19,4 \%)$ & \\
\hline & $>12 \mathrm{mg} / \mathrm{kgKg} / \mathrm{h}$ & $0(0,0 \%)$ & $2(13,3 \%)$ & $0(0,0 \%)$ & $2(6,5 \%)$ & \\
\hline \multirow{4}{*}{$\begin{array}{l}\text { 42) Komplikationen bei Propo- } \\
\text { folgabe }\end{array}$} & & $\mathrm{n}=20 / 21$ & $\mathrm{n}=20 / 23$ & $\mathrm{n}=3 / 6$ & $\mathrm{n}=43 / 50$ & \\
\hline & sehr selten $<1 \%$ & $14(70,0 \%)$ & $13(65,0 \%)$ & $2(66,7 \%)$ & $29(67,4 \%)$ & 0,965 \\
\hline & selten $1-10 \%$ & $4(20,0 \%)$ & $5(25,0 \%)$ & $1(33,3 \%)$ & $10(23,3 \%)$ & \\
\hline & häufig $>10 \%$ & $2(10,0 \%)$ & $2(10,0 \%)$ & $0(0,0 \%)$ & $4(9,3 \%)$ & \\
\hline \multirow[t]{10}{*}{ 43) Art der Komplikation } & & $\mathrm{n}=17 / 21$ & $n=15 / 23$ & $\mathrm{n}=1 / 6$ & $\mathrm{n}=33 / 50$ & \\
\hline & keine Relevanten & $3(17,7 \%)$ & $1(6,7 \%)$ & $0(0,0 \%)$ & $4(12,1 \%)$ & 0,593 \\
\hline & PRIS & $7(41,2 \%)$ & $7(46,7 \%)$ & $0(0,0 \%)$ & $14(42,4 \%)$ & 0,651 \\
\hline & verfärbter Urin & $2(11,8 \%)$ & $2(13,3 \%)$ & $0(0,0 \%)$ & $4(12,1 \%)$ & 0,923 \\
\hline & Hyperlipidämie & $2(11,8 \%)$ & $2(13,3 \%)$ & $0(0,0 \%)$ & $4(12,1 \%)$ & 0,923 \\
\hline & Niereninsuffizienz & $1(5,8 \%)$ & $1(6,7 \%)$ & $0(0,0 \%)$ & $2(6,1 \%)$ & 0,963 \\
\hline & Hypotonus & $6(35,3 \%)$ & $8(53,3 \%)$ & $0(0,0 \%)$ & $14(42,4 \%)$ & 0,402 \\
\hline & Atemdepression & $0(0,0 \%)$ & $1(6,7 \%)$ & $0(0,0 \%)$ & $1(3,0 \%)$ & 0,539 \\
\hline & Rhabdomyolyse & $1(5,8 \%)$ & $1(6,7 \%)$ & $0(0,0 \%)$ & $2(6,1 \%)$ & 0,963 \\
\hline & CK Erhöhung & $0(0,0 \%)$ & $2(13,3 \%)$ & $1(100,0 \%)$ & $3(9,1 \%)$ & 0,002 \\
\hline \multirow[t]{4}{*}{ 44) Dauer der Propofolgabe } & & $\mathrm{n}=20 / 21$ & $n=17 / 23$ & $n=2 / 6$ & $\mathrm{n}=39 / 50$ & \\
\hline & maximal 3 Tage & $2(9,9 \%)$ & $5(29,4 \%)$ & $0(0,0 \%)$ & $7(17,9 \%)$ & 0,392 \\
\hline & maximal 7 Tage & $14(70,0 \%)$ & $10(58,8 \%)$ & $1(50,0 \%)$ & $25(64,1 \%)$ & \\
\hline & mehr als 7 Tage & $4(20,1 \%)$ & $2(11,8 \%)$ & $1(50,0 \%)$ & $7(17,9 \%)$ & \\
\hline
\end{tabular}




\subsubsection{Beendigung der Sedierung}

Bei der Frage nach dem Vorgehen der Sedierungsreduktion antworteten die Kliniken uneinheitlich. So gaben jeweils $n=8 / 31(26 \%)$ der Kliniken an, die Sedierung um 50\% oder 20\% der Maximaldosis oder an einer klinischen Untersuchung orientierend zu reduzieren. Bei der Frage nach der Beendigung der Sedierung hingegen antworteten die Kliniken einheitlicher. Hier gaben $n=21 / 28(75 \%)$ der Kliniken an eine langsame Reduktion durchzuführen. Jeweils $\mathrm{n}=3 / 28(11 \%)$ gaben an sich an dem ICP oder an der Beatmungsentwöhnung (Weaning) zu orientieren (siehe Tabelle 21).

Tabelle 21: Reduktion und Beendigung der Sedierung

\begin{tabular}{|c|c|c|c|c|c|c|}
\hline Frage & Antwortmöglichkeiten & UH & $\operatorname{HVC}(\geq 30 / \mathrm{J})$ & $\operatorname{LVC}(<30 / \mathrm{J})$ & Gesamtantwor- & p-Wert \\
\hline \multirow{2}{*}{$\begin{array}{l}\text { 53) Reduktion der Sedie- } \\
\text { rung }\end{array}$} & & $\mathrm{n}=15 / 21$ & $n=15 / 23$ & $\mathrm{n}=1 / 6$ & $\mathrm{n}=31 / 50$ & \\
\hline & $50 \%$ & $6(40,0 \%)$ & $2(13,3 \%)$ & $0(0,0 \%)$ & $8(25,8 \%)$ & 0,103 \\
\hline \multirow[t]{4}{*}{ (\% der Maximaldosis) } & $30 \%$ & $1(6,7 \%)$ & $3(20,0 \%)$ & $0(0,0 \%)$ & $4(12,9 \%)$ & \\
\hline & $20 \%$ & $3(20,0 \%)$ & $5(33,3 \%)$ & $0(0,0 \%)$ & $8(25,8 \%)$ & \\
\hline & $10 \%$ & $1(6,7 \%)$ & $1(6,7 \%)$ & $1(100,0 \%)$ & $3(9,7 \%)$ & \\
\hline & Klinische Untersuchung & $4(26,7 \%)$ & $4(26,7 \%)$ & $0(0,0 \%)$ & $8(25,8 \%)$ & \\
\hline \multirow{5}{*}{$\begin{array}{l}\text { 73) Beendigung der Se- } \\
\text { dierung }\end{array}$} & & $\mathrm{n}=12 / 21$ & $n=15 / 23$ & $n=1 / 6$ & $\mathrm{n}=28 / 50$ & \\
\hline & Langsame Reduktion & $11(91,7 \%)$ & $10(66,7 \%)$ & $0(0,0 \%)$ & $21(75,0 \%)$ & 0,080 \\
\hline & ICP kontrolliert & $0(0,0 \%)$ & $2(13,3 \%)$ & $1(100,0 \%)$ & $3(10,7 \%)$ & \\
\hline & Weaning kontrolliert & $1(8,3 \%)$ & $2(13,3 \%)$ & $0(0,0 \%)$ & $3(10,7 \%)$ & \\
\hline & Ausschalten des Perfusors & $0(0,0 \%)$ & $1(6,7 \%)$ & $0(0,0 \%)$ & $1(3,6 \%)$ & \\
\hline
\end{tabular}




\subsection{Unerwünschte Nebenwirkungen der Analgosedierung}

Die Zentren wurden auch nach der Inzidenz der VST-assoziierten Komplikationen befragt. Diese Kategorie des Fragebogens umfasste die absolute Anzahl von beobachteten und dokumentierten Nebenwirkungen.

Von den $n=39 / 50(78 \%)$ der erhaltenen Antworten, berichteten $n=18 / 39(46 \%)$ der Zentren von häufig auftretenden Komplikationen ( $>10 \%)$, wobei $n=17 / 39(44 \%)$ von seltenem Auftreten $(1-10 \%)$, und $n=4 / 39(10 \%)$ von einem sehr seltenen Auftreten $(<1 \%)$ von Komplikationen berichteten (Tabelle 22 und Abbildung 11).

Tabelle 22: Häufigkeit des Auftretens beobachteter Komplikationen

\begin{tabular}{ccccccc}
\hline \hline Frage & Antwortmöglichkeiten & UH & HVC $(\geq 30 / \mathrm{J})$ & LVC $(<30 / \mathrm{J})$ & Gesamtantwort & p-Wert \\
\hline 56) Häufigkeit der & & $\mathbf{n}=\mathbf{1 9 / 2 1}$ & $\mathbf{n}=\mathbf{1 9 / 2 3}$ & $\mathbf{n}=\mathbf{1 / 6}$ & $\mathbf{n}=\mathbf{3 9 / 5 0}$ & \\
Komplikationen & sehr selten $<1 \%$ & $2(10,5 \%)$ & $2(10,5 \%)$ & $0(0,0 \%)$ & $4(10,3 \%)$ & 0,524 \\
& selten $1-10 \%$ & $10(52,6 \%)$ & $1(100,0 \%)$ & $1(100,0 \%)$ & $17(43,6 \%)$ & \\
& häufig $>10 \%$ & $7(36,8 \%)$ & $11(57,9 \%)$ & $0(0,0 \%)$ & $18(46,2 \%)$ & \\
\hline \hline
\end{tabular}

Bei der Befragung, was als potentielle Komplikation während der VST gewertet wird, wurden $\mathrm{n}=38 / 50(76 \%)$ an Antworten abgegeben. Obstipation ( $\mathrm{n}=32 / 38,84 \%)$ und eine Erhöhung der Transaminasen $(\mathrm{n}=28 / 38,74 \%)$ sowie der Cholestaseparameter $(\mathrm{n}=23 / 38,61 \%)$ galten dabei als leichte Komplikation bei VST. Als schwere Komplikation wurden am häufigsten Ileus ( $\mathrm{n}=30 / 38,79 \%$ ), ventilations-assoziierte Pneumonie (VAP) $(\mathrm{n}=23 / 38,61 \%)$, und die Critical-Illness-Polyneuropathie (CIP) ( $n=23 / 38,61 \%$ ) eingestuft. Laparatomien bei einer blockierten Magendarmpassage kamen bei $\mathrm{n}=35 / 41$ (85\%) der Zentren (mit weniger als fünf Vorkommnissen bezogen auf die letzten 30 Patienten) vor. $\mathrm{Zu} \mathrm{n}=28 / 41$ (68\%) wurden Medikamente zur Stimulation der Magendarmpassage nur verabreicht, wenn bereits eine Obstipation vorlag (Tabelle 24). 
In den Antworten zur Klassifizierung des Deliris als entweder leichtgradige oder schwergradige Komplikation fiel ein interessanter Unterschied auf (Abbildung 11). Delirium wurde als leichte Komplikation von $n=10 / 38(26 \%)(p=0,581)$ angegeben, und in $n=16 / 38(42 \%)$ der Fälle als schwere Komplikation eingeordnet $(\mathrm{p}=0,496)$. Zur Veranschaulichung der Ergebnisse sei hier auf Tabelle 23 verwiesen. Es ergab sich hier eine Signifikanz ( $p=0,017)$ bei der Angabe der Zentren in der Gesamtschau, wer Delir als leichtgradige Komplikation und gleichzeitig als schwergradige Komplikation angab. Es zeigte sich eine Uneinigkeit bezüglich der Zuordnung des Delirs als entweder leichtgradige oder schwergradige Komplikation.

So gab ein Zentrum Delir sowohl als leichtgradige als auch als schwergradige Komplikation an und 13 Zentren enthielten sich der Einstufung des Deliris (Tabelle 24).

Tabelle 23: Wahrnehmung als schwere vs. leichte Komplikation

\begin{tabular}{|c|c|c|c|c|c|}
\hline & \multicolumn{5}{|c|}{ Delir als schwere Komplikation } \\
\hline & & ja & nein & Gesamt & p-Wert \\
\hline \multirow[t]{3}{*}{ Delir als milde Komplikation } & ja & 1 & 9 & $\mathrm{n}=10 / 38$ & 0,017 \\
\hline & nein & 15 & 13 & $\mathrm{n}=28 / 38$ & \\
\hline & Gesamt & $\mathrm{n}=16 / 38$ & $\mathrm{n}=22 / 38$ & $\mathrm{n}=38 / 50$ & \\
\hline
\end{tabular}

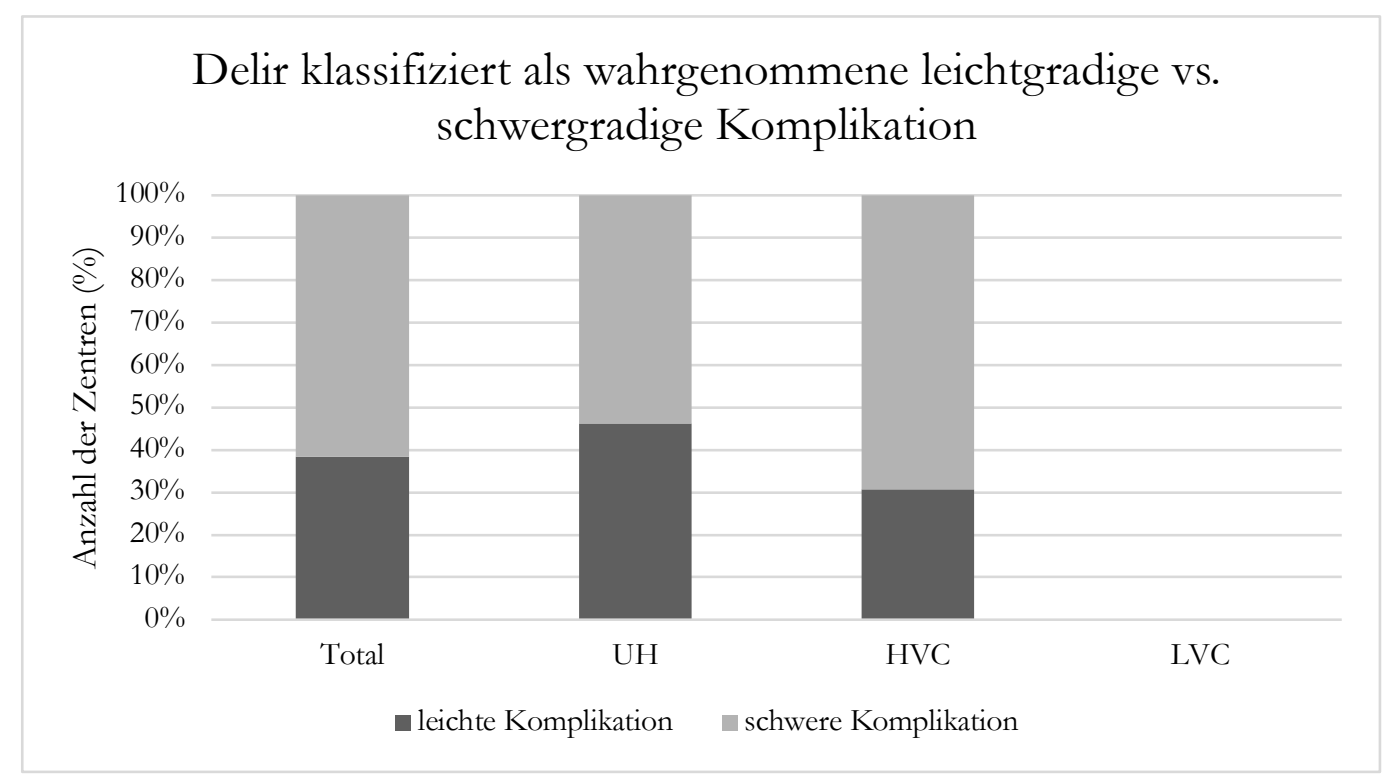

Abbildung 11: Antworten zur Klassifizierung eines Delirs. Zum Einen als wahrgenommene leichtgradige und zum Anderen als schwergradige Komplikation im Rahmen der Analgosedierung. Aufgeteilt nach Art des Zentrums (UH, HVC, LVC und ingesamt (Total)). 
Tabelle 24: Ergebnisse Komplikationen

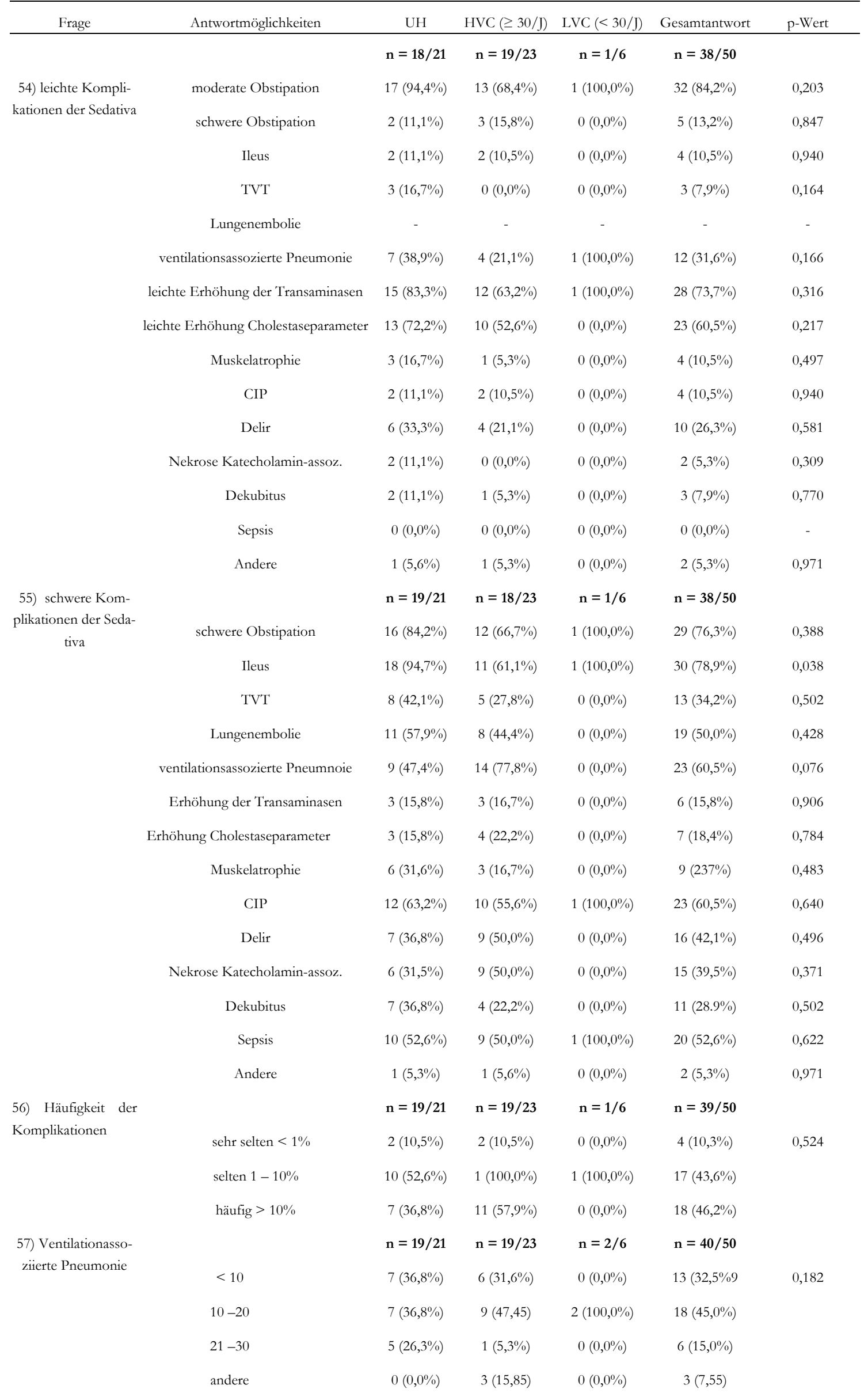




\begin{tabular}{|c|c|c|c|c|c|c|}
\hline Frage & Antwortmöglichkeiten & $\mathrm{UH}$ & $\operatorname{HVC}(\geq 30 / \mathrm{J})$ & $\operatorname{LVC}(<30 / \mathrm{J})$ & Gesamtantwort & p-Wert \\
\hline 58) Medikamente zur & & $\mathrm{n}=19 / 21$ & $\mathrm{n}=20 / 21$ & $\mathrm{n}=2 / 6$ & $\mathrm{n}=41 / 50$ & \\
\hline $\begin{array}{l}\text { Stimulation der Ma- } \\
\text { gendarmpassage }\end{array}$ & ja, immer & $8(42,1 \%)$ & $5(25,0 \%)$ & $0(0,0 \%)$ & $13(31,7 \%)$ & 0,318 \\
\hline \multirow{2}{*}{$\begin{array}{c}\text { nach Obstipation } \\
\text { durch Sedativa }\end{array}$} & ja, wenn Passage obstipiert & $11(57,9 \%)$ & $15(75,0 \%)$ & $2(100,0 \%)$ & $28(68,3 \%)$ & \\
\hline & niemals & $0(0,0 \%)$ & $0(0,0 \%)$ & $0(0,0 \%)$ & $0(0,0 \%)$ & \\
\hline \multirow{3}{*}{$\begin{array}{l}\text { 59) Anzahl der Lapa- } \\
\text { rotomien bei den } \\
\text { letzten } 30 \text { Patienten }\end{array}$} & & $\mathrm{n}=19 / 21$ & $\mathrm{n}=20 / 21$ & $\mathrm{n}=2 / 6$ & $\mathrm{n}=41 / 50$ & \\
\hline & weniger als $<5$ & $15(78,9 \%)$ & $18(90,0 \%)$ & $2(100,0 \%)$ & $35(85,4 \%)$ & 0,519 \\
\hline & keine & $4(21,1 \%)$ & $2(10,0 \%)$ & $0(0,0 \%)$ & $6(14,6 \%)$ & \\
\hline \multirow{5}{*}{$\begin{array}{l}\text { 60) Anzahl der Lun- } \\
\text { genembolien bei den } \\
\text { letzten } 30 \text { Patienten }\end{array}$} & & $\mathrm{n}=19 / 21$ & $\mathrm{n}=20 / 21$ & $\mathrm{n}=2 / 6$ & $\mathrm{n}=41 / 50$ & \\
\hline & $<5$ von 30 & $18(94,7 \%)$ & $17(85,0 \%)$ & $2(100,0 \%)$ & $37(90,2 \%)$ & 0,351 \\
\hline & $>5$ von 30 & $1(5,3 \%)$ & $0(0,0 \%)$ & $0(0,0 \%)$ & $1(2,4 \%)$ & \\
\hline & $>10$ von 30 & $0(0,0 \%)$ & $0(0,0 \%)$ & $0(0,0 \%)$ & $0(0,0 \%)$ & \\
\hline & keine & $0(0,0 \%)$ & $3(15,0 \%)$ & $0(0,0 \%)$ & $3(7,3 \%)$ & \\
\hline \multirow{5}{*}{$\begin{array}{l}\text { 61) Anzahl der er- } \\
\text { höhten Transa- } \\
\text { minase/ Cholestase- } \\
\text { parameter bei den } \\
\text { letzten } 30 \text { Patienten }\end{array}$} & & $\mathrm{n}=18 / 21$ & $n=20 / 23$ & $\mathrm{n}=2 / 6$ & $\mathrm{n}=40 / 50$ & \\
\hline & $<5$ von 30 & $3(16,7 \%)$ & $8(40,0 \%)$ & $0(0,0 \%)$ & $11(27,5 \%)$ & 0,452 \\
\hline & $>5$ von 30 & $9(50,0 \%)$ & $8(40,0 \%)$ & $1(50,0 \%)$ & $18(45,0 \%)$ & \\
\hline & $>10$ von 30 & $6(33,3 \%)$ & $4(20,0 \%)$ & $1(50,0 \%)$ & $11(27,5 \%)$ & \\
\hline & keine & $0(0,0 \%)$ & $0(0,0 \%)$ & $0(0,0 \%)$ & $0(0,0 \%)$ & \\
\hline \multicolumn{2}{|l|}{ 62) Hämodynami- } & $n=17 / 21$ & $n=20 / 23$ & $\mathrm{n}=2 / 6$ & $\mathrm{n}=39 / 50$ & \\
\hline \multirow{3}{*}{$\begin{array}{l}\text { sche Komplikationen } \\
\text { (\% der intubierten } \\
\text { Patienten) }\end{array}$} & $<10 \%$ & $2(11,8 \%)$ & $6(30,0 \%)$ & $2\left(100, B^{\circ} \%\right)$ & $10(25,6 \%)$ & 0,085 \\
\hline & $10-50 \%$ & $14(82,4 \%)$ & $12(60,0 \%)$ & $0(0,0 \%)$ & $26(66,7 \%)$ & \\
\hline & $>50 \%$ & $1(5,9 \%)$ & $2(10,0 \%)$ & $0(0,0 \%)$ & $3(7,7 \%)$ & \\
\hline \multirow{3}{*}{$\begin{array}{l}\text { 63) Komplikationen } \\
\text { durch zu tiefe Sedi- } \\
\text { eurng }\end{array}$} & & $\mathrm{n}=19 / 21$ & $\mathrm{n}=20 / 21$ & $\mathrm{n}=1 / 6$ & $\mathrm{n}=40 / 50$ & \\
\hline & ja & $9(47,4 \%)$ & $5(25,0 \%)$ & $0(0,0 \%)$ & $14(35,0 \%)$ & 0,260 \\
\hline & nein & $10(52,6 \%)$ & $15(75,0 \%)$ & $1(100,0 \%)$ & $26(65,0 \%)$ & \\
\hline \multirow{7}{*}{$\begin{array}{l}\text { 64) Art der Kompli- } \\
\text { kation }\end{array}$} & & $n=9 / 21$ & $n=5 / 23$ & $\mathrm{n}=0 / 6$ & $n=14 / 50$ & \\
\hline & PRIS & $4(44,4 \%)$ & $1(20,0 \%)$ & $0(0,0 \%)$ & $5(35,7 \%)$ & 0,453 \\
\hline & Sepsis & $2(22,2 \%)$ & $1(20,0 \%)$ & $0(0,0 \%)$ & $3(21,4 \%)$ & \\
\hline & Ileus & $2(22,2 \%)$ & $1(20,0 \%)$ & $0(0,0 \%)$ & $3(21,4 \%)$ & \\
\hline & Mydriasis & $1(11.1 \%)$ & $0(0,0 \%)$ & $0(0,0 \%)$ & $1(7,1 \%)$ & \\
\hline & Rhabdomyolyse & $0,0 \%$ & $1(20,0 \%)$ & $0(0,0 \%)$ & $1(7,1 \%)$ & \\
\hline & Darmischämie, Nekrose Akren & $0,0 \%$ & $1(20,0 \%)$ & $0(0,0 \%)$ & $1(7,1 \%)$ & \\
\hline \multirow{6}{*}{$\begin{array}{l}\text { 65) Mit welchem Me- } \\
\text { dikament assoziiert }\end{array}$} & & $\mathrm{n}=9 / 21$ & $\mathrm{n}=5 / 23$ & $\mathrm{n}=0 / 6$ & $n=14 / 50$ & \\
\hline & Propofol & $6(66,7 \%)$ & $4(80,0 \%)$ & $0(0,0 \%)$ & $10(71,4 \%)$ & 0,471 \\
\hline & Sufentanil & $1(11,1 \%)$ & $0(0,0 \%)$ & $0(0,0 \%)$ & $1(7,1 \%)$ & \\
\hline & Sevofluran & $1(11,1 \%)$ & $0(0,0 \%)$ & $0(0,0 \%)$ & $1(7,1 \%)$ & \\
\hline & Thiopental & $1(11,1 \%)$ & $0(0,0 \%)$ & $0(0,0 \%)$ & $1(7,1 \%)$ & \\
\hline & nicht ermittelbar & $0(0,0 \%)$ & $1(20,0 \%)$ & $0(0,0 \%)$ & $1(7,1 \%)$ & \\
\hline
\end{tabular}




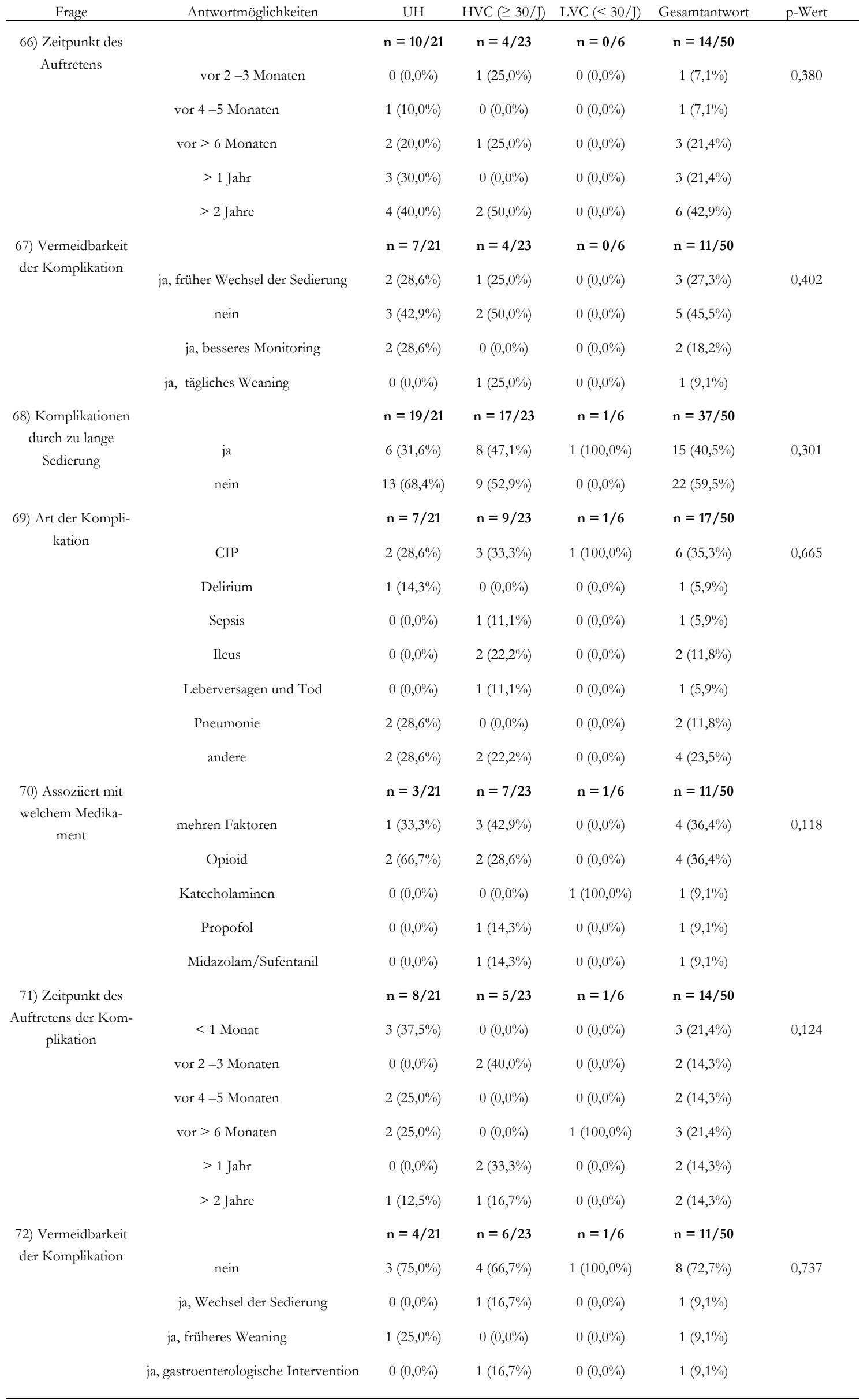




\subsection{Intensivmedizinisches Monitoring}

Bei der Auswertung der Dosierungen der verschiedenen Medikamente, muss stets in Betracht gezogen werden, dass trotz gleicher Infusionsrate eines Sedativums sich das Sedierungsniveau unterscheiden kann. Daher ist es besonders wichtig, die Sedierung mit objektivierbaren Methoden zu überwachen. Die Externalisierung und Objektivierung der Sedierungslevel wurde nur in $\mathrm{n}=21 / 42(50 \%)$ der Zentren mit technischen Hilfmitteln wie z. B. BIS oder einem EEG-Monitor (Narcotrend ${ }^{\circledR}$ ) durchgeführt (Abbildung 12). Narcotrend ${ }^{\circledR}$ wurde von $\mathrm{n}=5 / 20(25 \%)$ der HVC und sonst keinem Zentrum angewendet $(\mathrm{p}=0,044)$. Der BIS wurde von $n=16 / 42(38 \%)$ aller Zentren angewendet $(\mathrm{p}=0,582)$ (siehe Tabelle 25). Weitere Methoden, die als Freitextantwort angeben wurden, umfassten ein 6- bzw 12Kanal-EEG, sowie eine rein klinische Untersuchung.

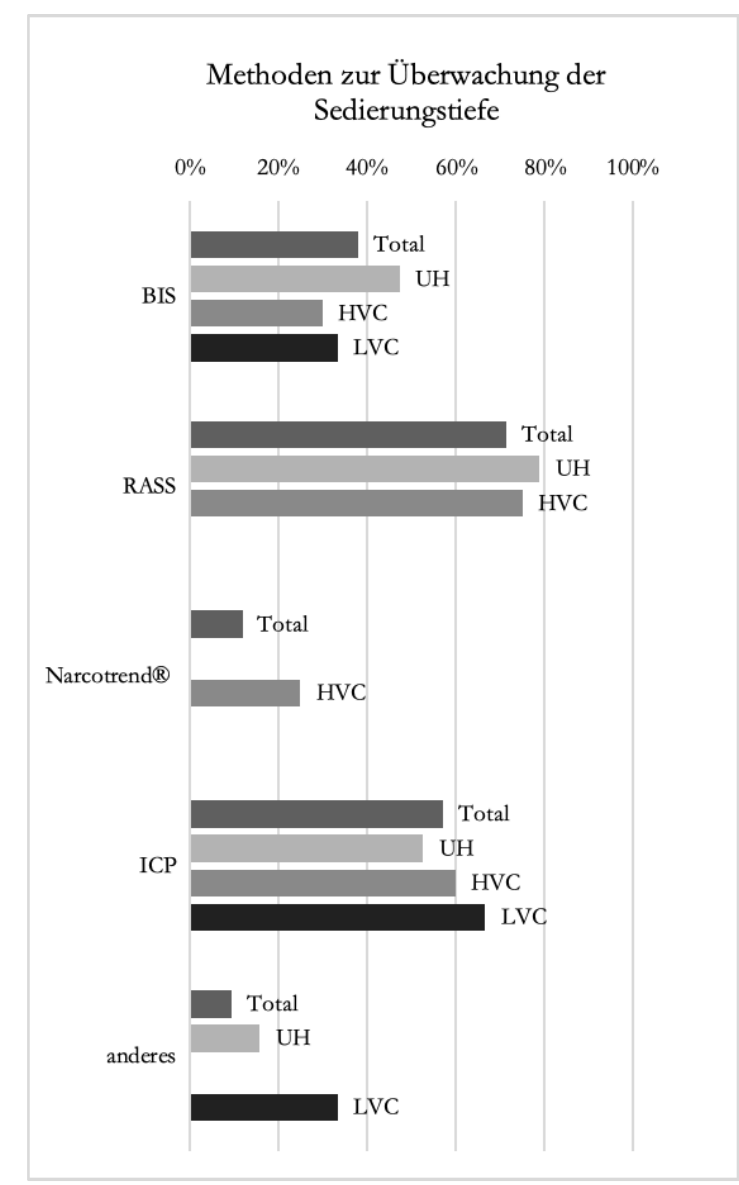

Abbildung 12: Objektivierung des Sedierungsniveaus. Aufgeteilt nach Art des Zentrums (UH, HVC, LVC und ingesamt (Total)). 
Bei der Überwachung der Sedierungstiefe vertrauten die meisten Zentren auf den Richmond agitation and sedation scale (RASS) $(n=30 / 42,71 \%)$ und den ICP-Wert $(n=24 / 42,57 \%)$ (siehe Tabelle 25). Eine invasive ICP- Überwachung wurde entweder über eine intraparenchymale ICP-Sonde $(\mathrm{n}=35 / 44,80 \%)$ und/oder über eine externe Ventrikeldrainage $(\mathrm{n}=39 / 44$, 89\%) durchgeführt. Die meisten Zentren steuerten den Blutdruck mit Blick auf den CPP (n = 37/41, 90\%), allerdings war ein Unterschied bei der Angabe des CPP Zielwerts zu beobachten, sowohl zwischen als auch innerhalb der Gruppen $(p=0,659)$ (Abbildung 13).

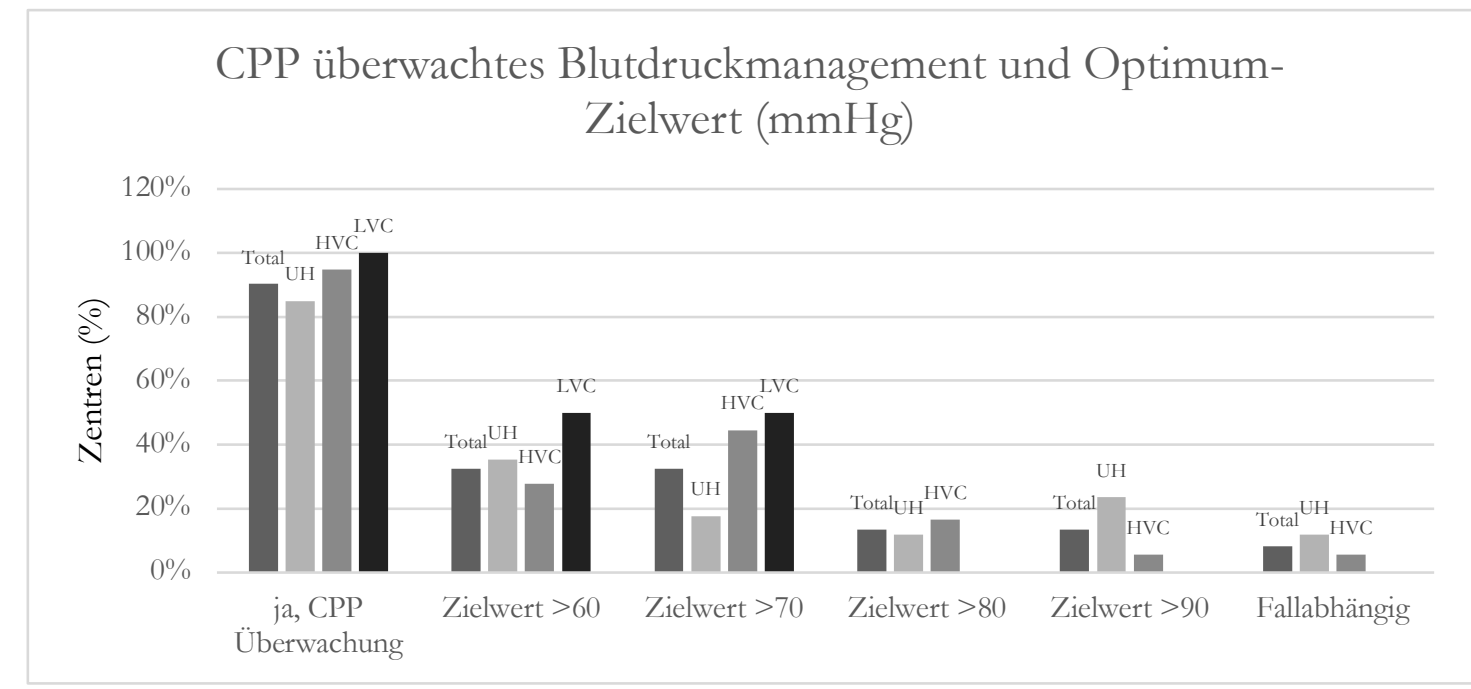

Abbildung 13: CPP-Überwachung . Regelmäßige CPP Überwachung und Angabe von CPP Zielwert zur Steuerung des Blutdrucks. Aufgeteilt nach Art des Zentrums (UH, HVC, LVC und ingesamt (Total)).

Die Anwendung von hämodynamischer Überwachung mittels pulse contour cardiac output (PiCCO) wurde mehrheitlich von $n=2 / 3$ (67\%) der LVC angewendet. Die UH ( $n=8 / 20$, 40\%) und HVC ( $\mathrm{n}=8 / 20,40 \%)$ nutzen PiCCO zu jeweils 40\%. Insgesamt gaben $\mathrm{n}=18 / 43$ $(42 \%)$ aller Kliniken an, diese Methode zu verwenden $(\mathrm{p}=0,665)$ (Tabelle 25).

Die Messung des zerebralen ptiO 2 , wurde deutlich häufiger von UH ( $\mathrm{n}=10 / 20,50 \%$ ), als von HVC ( $\mathrm{n}=3 / 20,15 \%)$ durchgeführt. Insgesamt $\mathrm{n}=13 / 43(30 \%)$ der Zentren gaben an, diese Methode zu verwenden. Hier fand sich auch eine statistische Signifikanz in den unterschiedlichen Angaben der Subgruppen ( $p=0,027)$. Insgesamt schien das invasive Monitoring weit verbreitet unter den UH und HVC zu sein, nicht dagegen in den LVC.

Die Einschätzung einer insuffizienten Sedierung wurde am häufigsten mithilfe einer klinischen Untersuchung gestellt. Diese führten $n=31 / 43$ (72\%) aller Zentren durch, gefolgt von der Diagnose anhand des ICP $\mathrm{n}=28 / 43(65 \%)$ und mittels BIS bzw. Narcotrend ${ }^{\circledR} \mathrm{n}=$ 22/43 (51\%). Zwischen den verschiedenen Zentren ließ sich kein signifikanter Unterschied ermitteln (siehe Tabelle 25). 


\section{Tabelle 25: Ergebnisse Monitoring}

\begin{tabular}{|c|c|c|c|c|c|c|}
\hline Frage & Antwortmöglichkeiten & $\mathrm{UH}$ & $\operatorname{HVC}(\geq 30 / \mathrm{J})$ & $\operatorname{LVC}(<30 / \mathrm{J})$ & Gesamtantwort & $\mathrm{p}$-Wert \\
\hline \multirow{6}{*}{$\begin{array}{l}\text { 31) Monitoring der Sedie- } \\
\text { rungstiefe }\end{array}$} & & $n=19 / 21$ & $n=20 / 23$ & $n=3 / 6$ & $\mathrm{n}=42 / 50$ & \\
\hline & BIS & $9(47,4 \%)$ & $6(30,0 \%)$ & $1(33,3 \%)$ & $16(38,1 \%)$ & 0,528 \\
\hline & RASS & $15(78,9 \%)$ & $15(75,0 \%)$ & $0(0,0 \%)$ & $30(71,4 \%)$ & 0,017 \\
\hline & Narcotrend $\AA$ & $0(0,0 \%)$ & $5(25,0 \%)$ & $0(0,0 \%)$ & $5(11,9 \%)$ & 0,044 \\
\hline & ICP & $10(52,6 \%)$ & $12(60,0 \%)$ & $2(66,7 \%)$ & $24(57,1 \%)$ & 0,845 \\
\hline & andere & $3(15,8 \%)$ & $0(0,0 \%)$ & $1(33,3 \%)$ & $4(9,5 \%)$ & 0,076 \\
\hline \multirow[t]{5}{*}{ 32) Messung des ICP } & & $\mathrm{n}=21 / 21$ & $n=20 / 23$ & $n=3 / 6$ & $n=44 / 50$ & \\
\hline & nicht gemessen & $1(4,8 \%)$ & $0(0,0 \%)$ & $0(0,0 \%)$ & $1(2,3 \%)$ & 0,571 \\
\hline & via ICP Sonde & $18(85,7 \%)$ & $18(90,0 \%)$ & $3(100,0 \%)$ & $35(79,5 \%)$ & 0,846 \\
\hline & Externe Ventrikeldrainage & $18(85,7 \%)$ & $14(70,0 \%)$ & $3(100,0 \%)$ & $39(88,6 \%)$ & 0,741 \\
\hline & andere Systeme & $2(9,5 \%)$ & $2(10,0 \%)$ & $0(0,0 \%)$ & $4(9,1 \%)$ & 0,850 \\
\hline \multirow{3}{*}{$\begin{array}{l}\text { 33) CPP kontrolliertes Blut- } \\
\text { druckmanagement }\end{array}$} & & $\mathrm{n}=20 / 21$ & $n=19 / 23$ & $n=2 / 6$ & $\mathrm{n}=41 / 50$ & \\
\hline & ja & $17(85,0 \%)$ & $18(94,7 \%)$ & $2(100,0 \%)$ & $37(90,2 \%)$ & \\
\hline & nein & $3(15,0 \%)$ & $1(5,3 \%)$ & $0(0,0 \%)$ & $4(9,8 \%)$ & 0,528 \\
\hline 34) Optimum CPP & & $\mathrm{n}=17 / 21$ & $n=18 / 23$ & $\mathrm{n}=2 / 6$ & $\mathrm{n}=37 / 50$ & \\
\hline \multirow[t]{5}{*}{$(\mathrm{mmHg})$} & $>60$ & $6(36,1 \%)$ & $5(27,8 \%)$ & $1(50,0 \%)$ & $12(32,4 \%)$ & 0,659 \\
\hline & $>70$ & $3(18,1 \%)$ & $8(44,4 \%)$ & $1(50,0 \%)$ & $12(32,4 \%)$ & \\
\hline & $>80$ & $2(10,9 \%)$ & $3(16,7 \%)$ & $0(0,0 \%)$ & $5(13,5 \%)$ & \\
\hline & $>90$ & $4(24,1 \%)$ & $1(5,6 \%)$ & $0(0,0 \%)$ & $5(13,5 \%)$ & \\
\hline & Fallabhängig & $2(10,9 \%)$ & $1(5,6 \%)$ & $0(0,0 \%)$ & $3(8,1 \%)$ & \\
\hline 35) Regelmäßige Pulsanalyse, & & $\mathrm{n}=20 / 21$ & $n=20 / 23$ & $\mathrm{n}=3 / 6$ & $\mathrm{n}=43 / 50$ & \\
\hline \multirow[t]{2}{*}{$\begin{array}{l}\text { Thermodilution, Pulmonararte- } \\
\text { rienkatheter, Echo (PiCCO) }\end{array}$} & ja & $8(40,0 \%)$ & $8(40,0 \%)$ & $2(66,7 \%)$ & $18(41,9 \%)$ & 0,665 \\
\hline & nein & $12(60,0 \%)$ & $12(60,0 \%)$ & $1(33,3 \%)$ & $25(58,1 \%)$ & \\
\hline \multirow{3}{*}{$\begin{array}{l}\text { 36) Regelmäßige Messung des } \\
\text { zerebralen ptiO2 }\end{array}$} & & $\mathrm{n}=20 / 21$ & $n=20 / 23$ & $\mathrm{n}=3 / 6$ & $n=43 / 50$ & \\
\hline & ja & $10(50,0 \%)$ & $3(15,0 \%)$ & $0(0,0 \%)$ & $13(30,2 \%)$ & 0,027 \\
\hline & nein & $10(50,0 \%)$ & $17(85,0 \%)$ & $3(100,0 \%)$ & $30(69,8 \%)$ & \\
\hline \multirow{5}{*}{$\begin{array}{l}\text { 37) Diagnose einer insuffizien- } \\
\text { ten Sedierung }\end{array}$} & & $\mathrm{n}=20 / 21$ & $n=20 / 23$ & $n=3 / 6$ & $n=43 / 50$ & \\
\hline & klinisch & $15(75,0 \%)$ & $13(65,0 \%)$ & $3(100,0 \%)$ & $31(72,1 \%)$ & 0,418 \\
\hline & 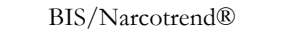 & $9(45,0 \%)$ & $11(55,0 \%)$ & $2(66,7 \%)$ & $22(51,2 \%)$ & 0,701 \\
\hline & ICP & $12(60,0 \%)$ & $13(65,0 \%)$ & $3(100,0 \%)$ & $28(65,1 \%)$ & 0,399 \\
\hline & andere z. B. EEG & $1(5,0 \%)$ & $0(0,0 \%)$ & $0(0,0 \%)$ & $1(2,3 \%)$ & 0,569 \\
\hline
\end{tabular}




\section{Diskussion}

Der Ergebnisteil weist eine Vielzahl von Informationen auf, welche in den folgenden Kapiteln diskutiert und in den aktuellen Forschungsstand eingeordnet werden. Ziel ist, aus den gewonnenen Daten und Erkenntnissen, Themen und Anstöße zur Erarbeitung standartisierter Leitlinien für das intensivmedizinische Management und den Einsatz der VST bei SAB herauszuarbeiten.

\subsection{Bedeutung der Ventilations- und Sedierungstherapie bei SAB- Patienten}

Während einerseits evidenzbasierte Leitlinien zum Verschluss von Aneurysmen und zur Prävention von DCI im Rahmen der SAB veröffentlicht wurden, mangelt es andererseits an evidenzbasierten Daten zum intensivmedizinischen Management bei SAB (Connolly et al. 2012). Die Neurocritical Care Society hat versucht, diese Lücken durch eine interdisziplinäre Konsens-Konferenz im Jahr 2011 zu schließen (Diringer et al. 2011). Dort wurde das Vorgehen bei DCI und systemischen Komplikationen adressiert, die detaillierten Aspekte der Intensivtherapie (z. B. die VST) fanden allerdings im Einzelnen keine Erwähnung. Das ist besonders alarmierend, da sich SAB-Patienten mit besonderen Herausforderungen und spezifischen Indikationen für eine VST präsentieren, die sich stark von den anderen Patienten auf Intensivstation unterscheiden (Citerio und Cormio 2003; Oddo et al. 2016).

Die VST ist von essentieller Bedeutung für die SAB-Patienten, als sie die zerebrale Sauerstoffextraktion $\left(\mathrm{CMRO}_{2}\right)$ verringert und somit das Missverhältnis zwischen Angebot und Nachfrage vermindert und damit die Ischämietoleranz des Hirngewebes erhöht (Oddo et al. 2016). Dazu kommen ihre essentielle Bedeutung bei der Therapie des ICP, die Verminderung des Sauerstoffverbrauchs des Hirns, die Reduktion der kortikalen Streudepolarisation (CSD) und Krampfanfallbehandlung.

Nichtsdestotrotz muss man der Vollständigkeit halber erwähnen, dass es im Hinblick auf das Outcome letztlich unter Umständen keinen Vorteil hat, wenn man beispielsweise durch eine tiefe Sedierung und eine optimierte Beatmung zwar eine DCI verhindert, aber der Patient an einer signifikanten VST-assozierten Komplikationen leidet. Hier lassen sich folgende Komplikationen aufführen, die aus einer zu langen oder zu tiefen Sedierung resultieren, wie z. B. kognitive Schäden oder chronische Herzinsuffizienz durch exzessive adrenerge Stimulation um den kardiodepressiven Wirkungen der Sedativa entgegen zuwirken (Oddo et al. 2016). 
Die vorliegende Arbeit bestätigt diese Lücke an Informationen zur Patientenversorgung mit VST, da die Umfragewerte gezeigt haben, dass fast die Hälfte aller SAB-Patienten eine VST bekommen (Kapitel 3.4, Tabelle 12).

Diese Dissertationsschrift zielt darauf ab, die verschiedenen VST-Standards in Deutschland zu charakterisieren. Das Hauptresultat ist, dass große Diskrepanzen in allen Bereichen offensichtlich sind. Die Hypothese konnte bewahrheitet werden. In den folgenden Abschnitten werden die wichtigsten Ergebnisse dieser Umfrage diskutiert und in Bezug zu den aktuellen Richtlinien für SAB gesetzt.

\subsection{Krankenhauseigenschaften}

Die erhobenen Daten lassen den Rückschluss zu, dass UH und HVC nicht nur mehr Erfahrung bei der Behandlung von SAB aufweisen, sondern auch, dass sie über mehr Ressourcen verfügen, um möglicherweise eine bessere bzw. umfassendere Patientenversorgung zu gewährleisten. Denn einerseits berichten die UH und HVC über höhere Fallzahlen von SABPatienten und andererseits über eine größere Vielzahl von zum Einsatz kommenden Methoden. In den abgefragten Merkmalen wie der Anzahl der interdisziplinären Visiten oder Oberarztvisiten, sowie dem Anteil der SAB-Patienten, die auf ICU behandelt werden, zeigen UH, HVC und LVC große Übereinstimmung.

Verschiedene Studien zeigen, dass das Langzeitergebnis nach einer akuten Hirnschädigung maßgeblich von der Erfahrung und Fallzahl des behandelnden Krankenhauses abhängt. Krankenhäuser, die mehr als 60 Patienten mit SAB im Jahr behandeln, haben beispielsweise eine signifikant geringere Rate an Morbidität und Mortalität (Cross et al. 2003; Nuño et al. 2012; McNeill et al. 2013).

Allerdings ist es nicht das Ziel dieser Umfrage herauszufinden, ob ein LVC eine größere Morbidität und Mortalität aufweist als ein UH und HVC bei der Behandlung von SAB. Die Umfrage wurde vielmehr ausgeführt, um aufzuzeigen, dass UH und HVC untereinander gröBere Übereinstimmungen in der Behandlung zeigen, als LVC, wie tatsächlich in dieser Studie beobachtet wurde.

Desweiteren berichten die LVC von keinem Einsatz von invasiven oder fortgeschrittenen Überwachungssystemen und komplexeren Sedierungstechniken, wie z. B. Inhalativa. Dieser Umstand kann auch an der geringen Datenmenge liegen. Ob diese Vorgehensweisen mit einem besseren Patientenüberleben einhergehen, muss noch in weiteren Studien herausgefunden werden. 
Leider konnte aufgrund der großen Heterogenität der Gruppengrößen nicht analysiert werden, ob sich Mixed-ICUs in ihren Behandlungsstrategien im Vergleich zu reinen Neuro-ICUs unterscheiden. Eine retrospektive Studie mit Patientendaten aus den Jahren 1990 bis 2005 hat jedoch gezeigt, dass die Prognose des Patienten nicht von der Spezialisierung der leitenden Fachrichtung der ICU beeinflusst wird. Dies ist dadurch bedingt, dass weder das fundierte Fachwissen der Intensivmediziner noch das spezielle Fachwissen der Neurochirurgen einen größeren Vorteil bietet (Mielke et al. 2019). Zukünftig wäre es allerdings interessant, die Unterschiede in der Leitung der ICU durch verschiedene Fachrichtungen in weiteren Studien zu analysieren.

\subsection{Beatmungsmanagement}

Die Ergebnisse dieser Umfrage zeigen, dass die tägliche Praxis der Beatmungstherapie von SAB-Patienten auf deutschen Intensivstationen sehr unterschiedlich ist. Der Mangel an evidenzbasierten Daten für die Erstellung allgemeingültiger Leitlinien betrifft auch die Beatmungstherapie bei SAB. Deutsche nationale Richtlinien für invasive Beatmung berücksichtigen jedoch nicht die besonderen Herausforderungen der Beatmung bei den SAB-Patienten (Baron et al. 2015). Eine der größten Herausforderungen stellt die eingeschränkte Evaluation des neurologischen Zustands des SAB-Patienten bei der Sedierung zur Beatmung dar ( $\mathrm{Pa}$ tienten mit hochgradiger SAB benötigen oft einen Atemwegsschutz aufgrund des Verlustes von Schutzreflexen und Bewusstseinsstörungen). Das Management von Komplikationen der Beatmung, wie z. B. die VAP stellt ebenfalls eine große Herausforderung dar. Die Richtlinien der Brain Trauma Foundation kommentieren zwar die Beatmungstherapie, jedoch nur für Patienten mit schwerem TBI (Carney et al. 2017). Beide Richtlinien können teilweise im Rahmen der Beatmungstherapie bei $\mathrm{SAB}$ anwendbar sein, es müssen aber immer die besonderen Herausforderungen und Besonderheiten von SAB-Patienten berücksichtigt werden (Tabelle 26) . 
Tabelle 26: Leitlinienempfehlungen zum Beatmungsmanagement

\begin{tabular}{|c|c|c|}
\hline Referenz & Empfehlung & Patientenpopulation \\
\hline \multirow[t]{3}{*}{$\begin{array}{l}\text { AWMF } \\
\text { (Baron et al. 2015) }\end{array}$} & $\begin{array}{l}\text { Eine Beatmungstherapie ist bei Patienten mit } \\
\text { schwerem ARDS (Horowitz-Index }<100 \mathrm{mmHg} \text { ) } \\
\text { indiziert. }\end{array}$ & $\begin{array}{l}\text { Akutes Lungenversa- } \\
\text { gen (ARDS) }\end{array}$ \\
\hline & $\begin{array}{l}\text { Anpassung der Beatmungseinstellungen für } \mathrm{paO}_{2} \\
60-80 \mathrm{mmHg} / \text { niedriger } \mathrm{FiO}_{2} \text { um druckindu- } \\
\text { zierte Lungenschädigung zu vermeiden. }\end{array}$ & ARDS \\
\hline & $\begin{array}{l}\text { Erwägen von permissiver Hyperkapnie }\left(\mathrm{paCO}_{2}>\right. \\
45 \mathrm{mmHg}) \text { um den maximalen Beatmungsdruck } \\
\text { zu reduzieren. Limitation: erhöhter ICP }\end{array}$ & ARDS \\
\hline \multirow[t]{2}{*}{$\begin{array}{c}\text { TBI } \\
\text { (Carney et al. 2017) }\end{array}$} & $\begin{array}{l}\text { Normale Beatmung ist das Ziel ohne zerebrale } \\
\text { Herniation sowie ein } \mathrm{paCO}_{2} \text { von } 35-45 \mathrm{mmHg} \text {. }\end{array}$ & Schweres TBI \\
\hline & $\begin{array}{l}\text { Eine verlängerte prophylaktische Hyperventila- } \\
\text { tion mit einem } \mathrm{paCO}_{2} \text { von } 25 \mathrm{mmHg} \text { oder weni- } \\
\text { ger wird nicht empfohlen. }\end{array}$ & Schweres TBI \\
\hline
\end{tabular}

Die Ergebnisse zeigen eine große Variabilität für die Indikation der Beatmungstherapie. 38\% der Zentren antworteten, dass ein Horowitz-Index von $<100 \mathrm{mmHg}$, der ein schwergradiges akutes Lungenversagen (ARDS) darstellt, die hausinterne Grenze für den Beginn einer Beatmungstherapie ist. Somit werden erst sehr spät Patienten intubiert, was höchstwahrscheinlich mit dem Verlust der neurologischen Überwachbarkeit begründet werden kann. Aufgrund des kritischen Zustands dieser Patienten mit einem Horowitz-Index $<100 \mathrm{mmHg}$, überlegen Experten oftmals, eine extrakorporale Membranoxygenierung (ECMO) durchzuführen (Paolone 2017; Patel et al. 2019). Die Mehrzahl der Zentren (61\%) wiederum gaben an, eine Beatmungstherapie bei einem Horowitz-Quotient von $<200 \mathrm{mmHg}$ frühzeitiger zu beginnen.

Im Fall von TBI empfehlen frühere Leitlinien, Hyperventilation als temporäre Maßnahme zur Reduktion von erhöhtem ICP bei der initialen Versorgung des Patienten (The Brain Trauma Foundation 2000; Firsching et al. 2015). Die jüngsten Richtlinien der The Brain Trauma Foundation für TBI haben dies jedoch überarbeitet und setzen sich derzeit für eine Normoventilation ein (Carney et al. 2017). Interessanterweise berichteten jedoch mehr als ein Drittel der befragten Zentren über Beatmungseinstellungen, dass sie keine Normoventilation, sondern einen stark erhöhten $\mathrm{paO}_{2}$ (> $100 \mathrm{mmHg}$ ) erreichen wollen. Kein LVC gab einen $\mathrm{paO}_{2}$-Zielwert an. 
Es gibt absolut keinen Beweis, dass ein $\mathrm{paO}_{2}>100 \mathrm{mmHg}$ neuroprotektiver wirkt als ein niedrigerer $\mathrm{paO}_{2}$, solange die Sauerstoffsättigung im physiologischen Bereich ist. Eine Hyperoxie kann allerdings einen Anstieg des ptiO2 bewirken. Allerdings entspricht diese Verbesserung des ptiO2 nicht unbedingt auch einer Verbesserung der Sauerstoffversorgung des Gehirns. Es kann zu einem besseren Gleichgewicht zwischen Sauerstoffzufuhr und -verbrauch des Hirngewebes im Falle einer leichten CBF-Reduktion kommen. Diese Verbesserung durch Hyperoxie ist allerdings bei einer starken CBF-Reduktion zu vernachlässigen (Rossi et al. 2000). Adäquate Oxygenierung spielt eine große Rolle bei DCI, aber ein hoher $\mathrm{paO}_{2}$ ist kein bewiesener Faktor für eine DCI Prophylaxe, da DCI multifaktorieller Pathophysiologie ist (Rowland et al. 2012; Sarrafzadeh et al. 2014). Dementsprechend heißt dies für die Beatmungstherapie, dass es trotz hoher $\mathrm{paO}_{2}$-Einstellung nicht unbedingt zu einer suffizienten Sauerstoffversorgung des Hirngewebes kommt und daher ein engmaschiges Monitoring wichtig ist.

Wenn dennoch hausinterne Standards bei der individuellen SAB-Therapie empfehlen, dass ein $\mathrm{paO}_{2}>100 \mathrm{mmHg}$ eingestellt sein sollte, könnte die strikte Einhaltung des Zielwerts zu viel invasiveren als nötigen Beatmungseinstellungen führen. Dieser Aspekt der per se „Übertherapie" würde die Schwelle für weitere Interventionen herabsetzen: wie beispielsweise von Röntgenexposition, bishin zu aggressiven Einstellungen vom inspiratorischem Spitzendruck, Tidalvolumina, Beatmungsfrequenz und invasiven Prozeduren wie Bronchoskopie und Thoraxdrainagen. Es ist jedoch fraglich, ob dieses Level von Invasivität gerechtfertigt ist, um strikt diesen Wert einzuhalten.

Gleiches gilt für die Einstellungen vom $\mathrm{paCO}_{2}$. Die meisten Zentren berichten über einen engen Korridor für den $\mathrm{paCO}_{2}$-Zielwert zwischen $35-45 \mathrm{mmHg}$. Der gesamte Bereich normwertiger $\mathrm{paCO}_{2}$-Werte $(35-45 \mathrm{mmHg})$ wird als prognostisch günstig bei mechanisch beatmeten SAB-Patienten angesehen wie es sich auch in der Umfrage widerspiegelt (Reiff et al. 2020). Eine retrospektive Studie an Patienten mit SAB ergab, dass Patienten mit $\mathrm{paCO}_{2-}$ Werten im hochnormalen Bereich (> $48 \mathrm{mmHg}$ ) eine schlechtere Prognose aufweisen als Patienten mit niedrigeren Werten (Yokoyama et al. 2018). Eine andere Studie allerdings zeigte jedoch potenzielle Vorteile einer kontrollierten Hyperkapnie bei Patienten mit SAB, denn ein $\mathrm{paCO}_{2}>60 \mathrm{mmHg}$ erhöht durch Vasodilatation nachweislich den CBF und verhindert damit möglicherweise eine DCI (Helbok et al. 2014).

Im Management von SAB ist das Verhindern von DCI und dadurch bedingten potentiellen ischämischen Defiziten äußerst wichtig. Unter normalen Bedingungen ist der $\mathrm{paCO}_{2}$ der stärkste Überwachungsparameter des CBF. 
Zwischen $20 \mathrm{mmHg}$ und $80 \mathrm{mmHg}$ reagiert der $\mathrm{CBF}$ linear auf den $\mathrm{paCO}_{2}$. Es gibt einen klaren Zusammenhang zwischen Hypoperfusion und zerebralem Vasospasmus.

Der CBF ist wichtig, um den Stoffwechselanforderungen des Gehirngewebes nachzukommen. Ein niedriger $\mathrm{paCO}_{2}$-Wert (z. B. durch Hyperventilation) kann durch Vasokonstriktion zu einem vermindertem CBF führen und die Komplikation der DCI verschlimmern, was die Anwendung der inzwischen veralteten TBI-Richtlinien für SAB potenziell gefährlich macht (Curley et al. 2010; Westermaier et al. 2016). Daher ist es sehr wichtig, das patientenindividuelle Verhältnis zwischen $\mathrm{paCO}_{2}$ und $\mathrm{CBF}$ zu berücksichtigen. Zusammenfassend kann also eine liberale Behandlung der Hyperkapnie unter der Überwachung des ICP protektiv für den Stoffwechsel des Hirngewebes sein, da sie zur Dilatation der Hirngefäße führt und damit den CBF erhöht (Petridis et al. 2010; Westermaier et al. 2014; Westermaier et al. 2016).

\subsection{Sedierungsmanagement}

In der Umfrage wurde angegeben, dass mindestens zwei oder mehr Medikamente benutzt wurden, um die Sedierung einzuleiten. Wenn eine verlängerte Sedierung (häufig für zehn bis vierzehn Tage) indiziert ist, z. B. bei verschlechtertem neurologischen Zustand, steigt die Gesamtzahl der angewendeten Sedativa enorm. Die spezifischen pharmakologischen Eigenschaften der eingesetzten Medikamente variieren, von Rezeptoraktivität und intraneuronalen Effekten bishin zu den unerwünschten klinischen Nebenwirkungen erheblich. Die tatsächliche Auswirkung der Vielzahl an Medikamenten auf Hirn und Organe ist bislang noch nicht bis zum Letzten untersucht und es werden immer wieder Wechselwirkungen beschrieben. So ist es bis jetzt nicht bekannt, welche Sedativa für die SAB-Patienten das optimale Profil besitzen (Oddo et al. 2016).

Die Umfragestudie zeigt eine große Vielfalt in der Wahl der Medikamente für die Einleitung, Aufrechterhaltung und Vertiefung der Sedierung, unabhängig von dem Behandlunsvolumen der Zentren auf. Allerdings können auch einige Gemeinsamkeiten in der Auswahl der Medikamente festgestellt werden. So wird Propofol von den meisten Zentren zur Einleitung und Ketamin zur Vertiefung der Sedierung genutzt.

Desweiteren zeigt diese Arbeit große Schwankungen der Medikamentendosierung. Wie die Ergebnisse zeigen, gibt es keinen Konsens bei der Maximaldosierung der zwei am häufigsten benutzen Analgosedativa Midazolam und Sufentanil. Das lässt Bedenken aufkommen, da bei beiden die zerebralen und systemischen Effekte sowie die unerwünschen Nebenwirkungen dosisabhängig sind. Außerdem geben die Zentren eine unterschiedliche Überwachung für das Detektieren von sedierungsassoziierten Komplikationen an. 
Diese Ergebnisse spiegeln die allgemeinen Studienlage wider, in welcher ebenso große Unterschiede beschrieben werden (Kapitel 1.4.2. und Tabelle 27).

All diese Ergebnisse sind vor dem Hintergrund zu erklären, dass generell noch viel Forschungsbedarf bei der Untersuchung der Eigenschaften, gewünschten Reaktionen und Komplikationen von den gewöhnlichen Sedativa besteht. Dies gilt auch, wenn die Medikamente bei SAB eingesetzt werden. Es ist nicht belegt, ob es einen dosis-abhängigen gewünschten Effekt oder einen Gegenteiligen gibt. Es darf nicht außer Betracht gelassen werden, dass es eine Akkumulation des Wirkstoffes im Patientenkörper gibt, der häufig 10 bis 14 Tage lang andauert (Devabhakthuni et al. 2012). Das lässt die Fragen zu, wie eine angepasste und individuellere Sedierungstherapie auszusehen hat, wie Komplikationen vermieden und wie der Behandlungsausgang bei SAB verbessert werden kann.

Im Rahmen von akuten Hirnschädigungen kommen Analgetika und Sedativa nicht nur zur Verhinderung von Agitation und Schmerz zum Einsatz, sondern auch aufgrund ihrer neuroprotektiven Eigenschaften. Diese Analgosedierung verringert die zerebrale Stoffwechselrate für Sauerstoff $\left(\mathrm{CMRO}_{2}\right)$, schränkt damit das Ungleichgewicht von Angebot und Nachfrage ein und erhöht somit die Toleranz des Hirngewebes für Ischämie (Oddo et al. 2016; Carney et al. 2017).

Zusätzlich konnte gezeigt werden, dass die Analgetika an Rezeptoren wirken, die in die Regulation von neuronaler Aktivität und synaptischer Übertragung involviert sind. Das könnte einen Einfluss auf CSD haben. CSD wird im Zusammenhang mit neurologischer Verschlechterung bei aktuen Hirnverletzungen und der Entstehung von DCI bei SAB gesehen (Hertle et al. 2012; Barr et al. 2013).

Studien zum intensivmedizinischen Management schlagen hingegen vor, dass das Minimieren der Sedierung die Prognose von intensivpflichtigen Patienten verbessern kann (Barr et al. 2013). Das wirft die Frage auf, wann die Indikation zur VST bei SAB besteht und wie diese einzuleiten und aufrechzuerhalten ist, und ob diese generelle Feststellung, dass eine zurückhaltende Sedierung gegebenfalls protektiv wirkt, auch für SAB-Patienten gilt. Die Datenlage zu den Eigenschaften und den dosisabhängigen Effekten und Nebenwirkungen der verschiedenen Sedativa beim Gebrauch im Rahmen einer SAB ist gering. In den folgenden Absätzen werden die vermuteten neuroprotektiven Eigenschaften der verschiedenen Sedativa und ihre Rolle bei der Behandlung der SAB diskutiert. 
Tabelle 27: Leitlinienempfehlungen zum Sedierungsmanagement

\begin{tabular}{|c|c|c|}
\hline Referenz & Empfehlung & Patientenpopulation \\
\hline \multirow[t]{5}{*}{$\begin{array}{l}\text { AWMF } \\
\text { (Baron et al. 2015) }\end{array}$} & $\begin{array}{l}\text { Eine ausreichende Analgesie und Sedierung ge- } \\
\text { mäß einem Ziel RASS sollte bei der Behandlung } \\
\text { von Patienten mit schwerer TBI und/oder intra- } \\
\text { kranieller Hypertonie durchgeführt werden. }\end{array}$ & TBI \\
\hline & $\begin{array}{l}\text { Ein Sedierungsregime mit Propofol oder Mida- } \\
\text { zolam ist gleichermaßen sicher. Wenn eine } \\
\text { schnelle neurologische Untersuchung erwünscht } \\
\text { ist, sollte Propofol bevorzugt werden. }\end{array}$ & TBI \\
\hline & $\begin{array}{l}\text { Bolusgaben von Opioiden (Sufentanil, Fentanyl, } \\
\text { Alfentanil) sollten bei Patienten mit TBI und IH } \\
\text { nur dann verabreicht werden, wenn der MAP } \\
\text { kontinuierlich überwacht und aufrechterhalten } \\
\text { wird, da sonst ein signifikanter Abfall der MAP } \\
\text { und der damit verbundene autoregulatorische An- } \\
\text { stieg des zerebralen Blutvolumens und ICP auf- } \\
\text { treten kann. }\end{array}$ & TBI \\
\hline & $\begin{array}{l}\text { Eine kontinuierliche intravenöse Verabreichung } \\
\text { von Opioiden (Remifentanil, Sufentanil, Fentanyl, } \\
\text { Morphin) sollte unter kontinuierlicher Blutdruck- } \\
\text { überwachung erfolgen. }\end{array}$ & TBI \\
\hline & $\begin{array}{l}\text { Aufgrund der günstigen Pharmakokinetik und da- } \\
\text { mit der Möglichkeit einer schnellen neurologi- } \\
\text { schen Beurteilung sollte Remifentanil anderen } \\
\text { Opioiden zur Analgesie und Sedierung vorgezo- } \\
\text { gen werden, sofern eine prozedurale Sedierung } \\
\text { nicht }>72 \text { Stunden erforderlich ist. }\end{array}$ & TBI \\
\hline $\begin{array}{c}\text { TBI } \\
\text { (Carney et al. 2017) }\end{array}$ & $\begin{array}{l}\text { Obwohl Propofol zur Kontrolle des ICP empfoh- } \\
\text { len wird, wird es nicht zur Verbesserung der Mor- } \\
\text { talität oder des 6-Monats-Outcome empfohlen. } \\
\text { Hochdosiertes Propofol kann eine signifikante } \\
\text { Morbidität hervorrufen. }\end{array}$ & TBI \\
\hline $\begin{array}{l}\text { European Stroke Organi- } \\
\text { sation } \\
\text { (Steiner et al. 2013) }\end{array}$ & $\begin{array}{l}\text { Keine Erwähnung von Sedierung, außer beim } \\
\text { Aneurysmenverschluss/ endovaskulären Inter- } \\
\text { vention und beim Patiententransfer nach Insult. }\end{array}$ & $\mathrm{SAB}$ \\
\hline
\end{tabular}




\subsubsection{Einleitung der Sedierung}

\subsubsection{Propofol}

Propofol ist in seiner Anwendung als Sedativum sehr beliebt, da es einen sehr schnellen Wirkungseintritt und eine kurze Wirkdauer hat, und somit einen breites Einsatzspektrum aufweist und beispielsweise für regelmäßige neurologische Untersuchungen (die tägliche Sedierungsunterbrechung (daily sedation interruption, DSI)) sehr geeignet ist. Über diese praktischen Vorteile hinaus wurde gezeigt, dass Propofol die neuroprotektiven Eigenschaften besitzt, die inhibitorischen Effekte der Gammaaminobuttersäure (GABA) zu potenzieren, und das Level von Endocannabinoiden zu erhöhen. Beides resultiert in einer weniger neurotoxischen Freisetzung von Glutamat (Adembri et al. 2007). Desweiteren hat Propofol eine antioxidative Wirkung. Bedingt ist dies einerseits durch die Erhöhung der Expression des antioxidativen Enzyms Hämoxygenase 1 (HO-1) in Astrozyten und andererseits durch direktes reagieren (scavenging) mit reaktiven Sauerstoff-Spezies (ROS) und inhibieren von Lipidperoxidation (Wilson und Gelb 2002). Propofol ist in dieser Umfrage das am häufigsten angewendete Medikament zur Einleitung und Aufrechterhaltung der Sedierung. In einer Studie von Skoglund et al. (2013) werden Propofol, wie auch Midazolam ebenfalls mehrheitlich als primäres Sedativum verwendet. Die Umfrage zeigt, dass Komplikationen im Rahmen der Propfolgabe insgesamt sehr selten beobachtet wurden bei einer mehrheitlichen Administration von maximal sieben Tagen. Als häufigste Komplikationen werden Hypotonus und das lebensbedrohliche PRIS angegeben.

\subsubsection{Midazolam}

Midazolam ist in dieser Umfrage ebenfalls als ein sehr häufig eingesetztes Medikament angegeben worden. Midazolam ist ein GABAerges Medikament der Benzodiazepingruppe und spielt eine etablierte Rolle im Management von erhöhtem ICP bei TBI (Carney et al. 2017). Dass Midazolam in der Leitlinie empfohlen wird und in der Umfragestudie häufig angegeben wird, legt die Vermutung nahe, dass Empfehlungen für TBI auf Patienten mit SAB übertragen werden.

Die Verwendung beider Medikamente - Midazolam und Propofol - wird in den aktuellsten Richtlinien für TBI erwähnt, aber die Datenlage reicht nicht aus, um eine eindeutige Empfehlung für den Einsatz aussprechen zu können (Carney et al. 2017). In den nationalen Richtlinien für VST werden beide Medikamente als gleichwertig betrachtet, aber die Verabreichung von Propofol wird allerdings gegenüber Midazolam empfohlen, wenn eine schnelle neurologische Beurteilung des Patienten gewünscht wird (Baron et al. 2015). 
Laut der retrospektiven Studie von Hertle et al. (2016) ist trotz mutmaßlicher neuroprotektiver Eigenschaften die Anwendung von GABAergen Sedativa bei SAB Patienen mit einem ungünstigem Ausgang nach sechs Monaten assoziert. Midazolam kann eine CSD induzieren, sowie die Energienutzung des Gehirns reduzieren. Diese Umstände deuten darauf hin, dass eine GABAerge vermittelte metabolische Depression zum Versagen energieabhängiger Ionenpumpen führt und somit zu einer CSD, und im weiteren Verlauf möglicherweise auch zu einer DCI führen kann (Hertle et al. 2016; Carney et al. 2017; von der Brelie et al. 2017). Die beiden Substanzen besitzen zudem kardiodepressive Eigenschaften, sodass eine adrenerge Stimulation mittels Vasopressoren nötig ist, um einen geeigneten CPP aufrechtzuerhalten (Oddo et al. 2016). Im Zusammenhang mit der Grunderkrankung einer SAB, bei welcher die Patienten häufig einer pharmakologisch induzierten Hypertension ausgesetzt sind, um eine DCI zu behandeln, ist dieser kardiodepressive Nebeneffekt der Benzodiazepine als besonders kontraproduktiv zu betrachten (Francoeur und Mayer 2016). Die Anwendung von Empfehlungen, die auf TBI-Patienten ausgerichtet sind, könnte somit gefährlich für SABPatienten sein.

\subsubsection{Sufentanil}

Ein weiteres Medikament, welches in dieser Umfrage abgefragt wurde, ist Sufentanil. Das schnell metabolisierte synthetische Opioid hat sich in der Neurointensivbehandlung aus den gleichen Gründen wie Propofol (wegen seiner kurzen Wirkungsdauer und zur Vertiefung der Sedierung) immer mehr durchgesetzt. Allerdings wurde Sufentanil mit einem fallenden mittleren arteriellen Druck (MAP) assoziiert (Bratton et al. 2007). Im Rahmen von SAB können MAP-Senkungen zu zerebraler Hypoperfusion führen und möglicherweise das Risiko für DCI erhöhen, wodurch dieses Narkotikum nicht gänzlich optimal für die Sedierungsaufrechterhaltung bei SAB-Patienten ist. Diese pharmakologischen Wirkungen von Opioiden werden in den nationalen Richtlinien in Bezug auf TBI und intrakranielle Hypertonie behandelt, bleiben aber im Bezug auf SAB-Patienten unerwähnt (Baron et al. 2015).

Um der kardiodepressiven Wirkung der Opioide entgegen zu wirken, kommen Vasopressoren (z. B. Noradrenalin) zum Einsatz. Die Vasopressoren können allerdings zu Lungenödemen, sowie zur akuten Herzinsuffizienz bei SAB-Patienten führen. Daher ist eine kontinuierliche Überwachung der Herzfunktion z. B. mittels transkardiopulmonalem hämodynamischen Monitoring notwendig (Manoel et al. 2016; Bederson et al. 2009).

Der Einsatz von Sedativa und weiteren Substanzen, wie Vasopressoren muss unbedingt unter engmaschigen Monitoring stattfinden. Kardiale Dysfunktionen und insbesondere die akute Kardiomyopathie gehören zu den schwerwiegendsten Komplikationen nach SAB. 
Diese stehen im Zusammenhang mit einer Dysfunktion des autonomen Nervensystems in Form einer exzessiven Ausschüttung von myokardialen Katecholaminen. Troponin I als Parameter der Myokardschädigung ist bei 20\% bis 30\% der SAB-Patienten erhöht (Seule et al. 2010)

Die Beliebtheit von Propofol und Sufentanil spiegelt sich auch in dieser Umfrage wider. Man könnte vermuten, dass diese Popularität auf ihre kurze Wirkungsdauer und die Fähigkeit zur Durchführung von DSI zurückzuführen ist, was auch in den nationalen Richtlinien als einer ihrer Vorteile erwähnt wird (Baron et al. 2015).

Allerdings ist die DSI bei SAB kritisch zu sehen und der häufige Einsatz von Propofol und Midazolam kann hinterfragt werden, da der positive Nutzen der Medikamente zur Durchführung einer DSI im Rahmen der SAB Behandlung keine Rolle spielt. In einer Metaanalyse, die den Einsatz von Sedierungsprotokollen im Vergleich zum Einsatz von DSI auswertet, wurden keine statistisch signifikanten Unterschiede hinsichtlich des Outcomes der Patienten beobachtet (Nassar und Park 2016). Bei der Neurointensivtherapie, und insbesondere bei SAB-Patienten, könnte die DSI regelmäßige neurologische Untersuchungen ermöglichen, um neu aufgetretende Defizite oder eine DCI frühzeitig zu erkennen. In einer prospektiven Studie von Helbok et al. (2012) zu 20 TBI Patienten unter DSI-Therapie wurden jedoch neurologische Defizite in nur 2\% der Aufwachversuche (wake-up trials) erkannt. Ein Drittel dieser Versuche musste wegen ICP-Krisen, Agitationen, oder systemischer Entsättigung, die zu kritischen Werten von $\mathrm{ptiO}_{2}$ und zerebralen Stoffwechselstörungen führten, abgebrochen werden. Analog erhielten Skoglund et al. (2009) in einer Studie mit neun SAB-Patienten während der DSI-Therapie signifikant erhöhte ICP- und CPP-Werte. Während die Autoren in ihrer Studie argumentieren, dass diese Anstiege nicht mit einer schlechteren Prognose der Patienten korrelieren, führt die geringe Anzahl der Patienten bei Betrachtung dieser Daten zu einem Selektionsbias. Somit stellt diese Studie einen schwachen Nachweis zur Begünstigung von DSI bei SAB dar. Zusammenfassend zeigen diese Studien also, dass eine DSI bei SAB nicht unbedingt zur Identifizierung neuer neurologischer Defizite führt, und diese Praxis mit ICP-Krisen, Agitation und systemischem Sauerstoffabfall verbunden ist, die wiederum zu zerebralen Stoffwechselbelastungen führen (Skoglund et al. 2009; Helbok et al. 2012). Folglich gibt es keine Übereinstimmung was die Durchführung von DSI bei SAB-Patienten mit VST angeht. Daher können die theoretischen Vorteile eines wachen Patienten zur neurologischen Beurteilung im Konflikt zu den positiven Aspekten der Sedierung stehen. 


\subsubsection{Vertiefung der Sedierung}

Die Umfrage zeigt eine große Vielfalt in der Wahl der Medikamente zur Induktion der Sedierung, der Aufrechterehaltung und der Vertiefung, ganz unabhänging von der Fallzahl der SAB-Patienten der Zentren. Die neuroprotektiven Eigenschaften der Sedativa durch verschiedene Mechanismen und der gleichzeitige Mangel an einem hohen Evidenzgrad, um eine Sedierung in der Neurointensivtherapie durchzuführen, ist wahrscheinlich der Grund für die in dieser Arbeit beobachteten Unterschiede. Verschiedene Autoren haben einen praktischen Ansatz zur Sedierung - angepasst auf die speziellen Bedürfnisse eines SAB-Patienten - unter der Beachtung des Schweregrads des Hirnschadens und der Werte der multimodalen Überwachung empfohlen, um die therapeutischen Ziele zu erreichen (Nuño et al. 2012).

\subsubsection{Ketamin}

Bei der Befragung zur Vertiefung der Sedierung wird Ketamin von den meisten UH und HVC eingesetzt. Ketamin, ein atypisches Sedativum und Analgetikum, wirkt als nicht-kompetitiver N-Methyl-D-Aspartat (NMDA) Rezeptorantagonist. In den letzten Jahren hat Ketamin sich zu einem sehr attraktiven Medikament für VST bei TBI und SAB entwickelt. Eine prospektive, randomisierte Studie zu Ketamin mit zehn TBI Patienten und acht SAB-Patienten, zeigt eine effektive Inhibition der CSD (Carlson et al. 2018). Zusätzlich konnte in einer retrospektiven Studie gezeigt werden, dass Ketamin mit einer niedrigeren Inzidenz von DCI-assozierten Infarkten und niedrigerem ICP in SAB-Patienten vergesellschaftet ist (von der Brelie et al. 2017). Zusammengenommen deuten diese beiden Studien einen vorteilhaften Einfluss von Ketamin auf SAB-Patienten an. Die nationalen AWMF-Leitlinien beschreiben den routinemäßigen Einsatz von Ketamin bei Patienten mit TBI und intrakranieller Hypertonie (Baron et al. 2015).

Nur LVC bevorzugen Clonidin zur Vertiefung der Sedierung. Clonidin ist ein alpha-2-Adrenozeptor-Agonist, hemmt die Freisetzung von Noradrenalin und Adrenalin und wirkt somit antihypertensiv. Desweiteren besitzt Clonidin analgetische, sedative und anxiolytische Eigenschaften. Clonidin findet allerdings kaum Erwähnung in den nationalen Leitlinien und die generelle Datenlage für den Einsatz auf Intensivstation ist sehr spärlich Jamadarkhana und Gopal 2010). Die nachstehende Tabelle (Tabelle 28) zeigt Empfehlungen für Ketamin zur Vertiefung der Sedierung. Die meisten Empfehlungen haben keinen verbindlichen Charakter. 
Tabelle 28: Leitlinienempfehlungen zum Sedierungsmanagement

\begin{tabular}{|c|c|c|}
\hline Referenz & Empfehlung & Patientenpopulation \\
\hline \multirow[t]{4}{*}{$\begin{array}{c}\text { AWMF } \\
\text { (Baron et al. 2015) }\end{array}$} & $\begin{array}{l}\text { Ketamin-Racemat sollte bei Patienten mit TBI } \\
\text { und } \mathrm{IH} \text { unter kontrollierter Beatmung (konstanter } \\
\mathrm{paCO}_{2} \text { ) und zusätzlich zum GABA-Rezeptor-A- } \\
\text { gonisten (Blockade der exzitatorischen Potenzi- } \\
\text { ale) in Betracht gezogen werden. }\end{array}$ & TBI \\
\hline & $\begin{array}{l}\text { Durch Ketamin-Racemat (sympathomimetische } \\
\text { und positive hämodynamische Wirkung) kann } \\
\text { eine klinisch relevante Reduktion von MAP und } \\
\text { CPP vermieden werden. }\end{array}$ & TBI \\
\hline & $\begin{array}{l}\text { Sowohl ein Ketamin-Racemat/Midazolam-basie- } \\
\text { rendes oder ein auf Opioid/Midazolam-basieren- } \\
\text { des Sedierungsregime kann bei mechanisch beat- } \\
\text { meten Patienten mit TBI und IH eingesetzt wer- } \\
\text { den (kein signifikanter Unterschied in der Wir- } \\
\text { kung auf ICP und CPP). }\end{array}$ & TBI \\
\hline & $\begin{array}{l}\text { Ein Esketamin/ Methohexital-basierendes und } \\
\text { ein Fentanyl/Methohexital-basierendes Sedie- } \\
\text { rungsregime kann gleichermaßen sicher (in Bezug } \\
\text { auf ICP und CPP) und effektiv (in Bezug auf die } \\
\text { erreichte Sedierung) bei mechanisch beatmeten } \\
\text { Patienten mit TBI und IH eingesetzt werden. }\end{array}$ & TBI \\
\hline $\begin{array}{c}\text { TBI } \\
\text { (Carney et al. 2017) }\end{array}$ & $\begin{array}{l}\text { Die Verabreichung von hochdosiertem Barbiturat } \\
\text { wird empfohlen, um einen erhöhten ICP zu kon- } \\
\text { trollieren und gleichzeitig eine medizinische und } \\
\text { chirurgische Behandlung auf höchstem Niveau zu } \\
\text { ermöglichen. Hämodynamische Stabilität ist uner- } \\
\text { lässlich vor und während der Barbiturattherapie. }\end{array}$ & TBI \\
\hline
\end{tabular}

\subsubsection{Barbiturate}

Die Einleitung einer Sedierung durch Barbiturate bei SAB-Patienten ist nicht üblich. Die Empfehlungen leiten sich von Studien ab, in welchen verschiedene Patientenkollektive mit TBI und intrakranieller Hypertension analysiert wurden (Dereeper et al. 2002; Kim et al. 2008). Diese haben gezeigt, dass Barbiturate einen refraktären symptomatischen Vasospasmus reduzieren (Finfer et al. 1999; Heo et al. 2003). Sie unterstreichen somit die mögliche Rolle von Barbituraten im Management von SAB über die reine ICP-Kontrolle hinaus. 
Ein großer Nachteil im Umgang mit Barbituraten ist das relativ häufige Auftreten von Komplikationen, wie eine verlängere Sedierung, Stoffwechselstörungen, Atemdepression (durch zentrale Atemlähmung bei geringer therapeutischer Breite) und kardiovaskuläre Ereignisse (Alotaibi et al. 2017). In der Umfrage zeigt sich, dass die meisten UH und HVC Barbiturate zur Vertiefung der Sedierung einsetzen, während LVC diese scheinbar eher zögerlich verwenden. Dies könnte entweder an der geringen Anzahl der Befragten liegen, oder auch eine geringere Erfahrung bzw. weniger Ressourcen widerspiegeln, um Komplikationen zu begegnen. Der Einsatz von Barbituraten ist jedoch der am meisten untersuchte Aspekt der VST in der Neurointensivtherapie, und die aktuellen TBI-Richtlinien können zumindest eine Empfehlung zur Sedierungsverbesserung mit diesen Medikamenten abgeben, wenn Patienten an refraktärer intrakranieller Hypertonie leiden (Carney et al. 2017)(siehe Tabelle 28).

\subsubsection{Isofluran und Sevofluran}

Ein weiteres Ergebnis der Studie ist, dass nur ein UH über den Einsatz von Isofluran und Sevofluran als Mittel der ersten Wahl zur Aufrechterhaltung der Sedierung berichtet. Obwohl keine statistische Signifikanz zu beobachten ist, lässt sich dennoch ein Trend zu einem häufigeren Gebrauch von volatiler Sedierung bei UH im Gegensatz zu HVC und LVC ablesen. Dieser Umstand betont die experimentelle und noch weiter zu bewertende Stellung der volatilen Sedierung in der Behandlung der SAB. Bestärkt wird diese Aussage durch die Ergebnisse bezüglich Modalitäten zur Administration von Sevofluran und Isofluran. Die Systeme werden von knapp über 50\% derjenigen Zentren benutzt, die diese Frage beantwortet haben. Die Erfahrung wurde als überwiegend gut angeben. Allerdings lassen die Ergebnisse vermuten, dass diese Methode kein weit verbreitetes Standardverfahren ist.

In einer großen prospektiven Studie zu einer Serie von 25 Patienten mit akuten Schlaganfall oder SAB wurde bei einem Drittel der Patienten eine Sedierung mit Sevofluran mit einigen schwerwiegenden Komplikationen in Zusammenhang gebracht. Bei diesen Komplikationen handelte es sich um eine CPP-Verminderung und vorübergehende ICP-Erhöhung, die wahrscheinlich durch die vasodilatatorischen Effekte der Hyperkapnie entstanden sind (Purrucker et al. 2015). Bei SAB-Patienten ohne erhöhtem ICP verbesserte Isofluran allerdings signifikant den zerebralen Blutfluss im Vergleich zu Propofol und zeigte sich somit als vorteilhaft im Rahmen der Prävention von DCI (Villa et al. 2012). 


\subsection{Unerwünschte Nebenwirkungen der Analgosedierung}

In dieser Studie wurde u. a erfragt, ob die teilnehmenden Zentren Komplikationen im Zusammenhang mit der VST beobachten haben, z. B. im Rahmen der Gabe eines bestimmten Medikamentes oder generell durch zu tiefe oder zu lange Sedierung. Zudem wurde erfragt, ob und als wie bedeutend diese Komplikationen wahrgenommen wurden.

Die Daten zeigen auch bei diesem Themenkomplex eine große Differenz. Einige Komplikationen im Zusammenhang mit VST werden von einigen Zentren als selten auftretend klassifiziert und wiederum in anderen Krankenhäusern als häufig auftretende Komplikationen angegeben. Obstipation, Erhöhung der Transaminasen und Cholestaseparameter sind die am häufigsten beobachteten Komplikationen, die als leichtgradig klassifiziert werden. Ileus, VAP und Critical-Illness Polyneuropathie (CIP) gehören zu den am häufigsten beobachteten und als schwergradig klassifizierten Komplikationen. Die Daten lassen den Rückschluss zu, dass der Auslöser der jeweiligen Komplikation im klinischen Alltag noch genauer identifiziert werden muss, um das Auftreten der Komplikation besser verhindern zu können.

Ein weiteres, sehr interessantes Ergebnis dieser Arbeit ist, dass viele Zentren das Delir, ein akutes, komplexes, hirnorganisches Syndrom mit Störungen des Bewusstseins, der kognitiven Funktionen, der Psychomotorik, des Schlaf-Wach-Rhythmus und der Emotionalität, nicht als schwergradige Komplikation bei VST der SAB-Patienten wahrnehmen. Dabei ist bei allgemeinen Intensivpatienten ein Delir mit einer dreifach erhöhten Sterblichkeit innerhalb von sechs Monaten, sowie einer permanenten kognitiven Verschlechterung assoziiert (Ely et al. 2004; Jackson et al. 2004).

Es ist im Zusammenhang mit dem Krankheitsbild der SAB unklar, ob das Delir eine Komplikation der Intensivtherapie, oder eine Begleiterscheinung des Schweregrads der Erkrankung darstellt (Caeiro et al. 2005; Sauvigny et al. 2019). Ungeachtet seiner Ursache, wird das Delir im Zusammenhang mit der Entstehung verschiedenster Komplikationen im Verlauf der Intensivtherapie bei SAB-Patienten sowie dem möglichem Einfluss auf langfristige Therapieerfolge gesehen (Reznik et al. 2017).

Daher wirft das Unterschätzen des Delirs, als mögliche Komplikation der VST, die Frage auf, ob es verlässlich genug diagnostiziert und anschließend behandelt wird. Zusammengenommen zeigt die unterschiedliche Einschätzung des Delirs, dass möglicherweise das Management von VST-assozierten Komplikationen weiter standartisiert werden muss. Dieser Umstand kann auch im Zusammenhang mit der generellen Frage nach der Herkunft der Komplikation und den auslösenden Faktoren gesehen werden. 
Manifestiert sich z. B. ein Delir bei einem SAB-Patienten in den ersten Tagen nicht durch eine klinische Verschlechterung, sondern durch eine mangelnde Verbesserung des neurologischen Zustands. Analog dazu wird z. B. von einem Patienten erwartet, dass er sich von der anfänglichen Ischämie nach einem zerebralen Vasopasmus erholt. Zeigt der Patient aber keine Erholung, da sich beispielsweise ein akuter Hydrozephalus entwickelt hat, verhindert dieser jede mögliche Verbesserung (Rinkel und Klijn 2009).

\subsection{Erweitertes intensivmedizinisches Monitoring}

Was die erweiterte Überwachung der SAB-Patienten betrifft, halten sich die meisten der befragten Klinken an die international etablierten Empfehlungen. In Übereinstimmung mit der International Multidisciplinary Consensus Conference on Multimodality Monitoring (IMCCMM) führen die meisten der befragten Zentren ein ICP-Monitoring mit einer intraparenchymalen Sonde und/oder externen Ventrikeldrainage (EVD) durch (Helbok et al. 2014; Roux et al. 2014).

Zusätzlich verwenden die meisten der befragten Zentren RASS, eine zehnstufige Skala zum Monitoring der Sedierungstiefe von SAB-Patienten. RASS ist das einzige diagnostische Instrument, das sowohl die Sedierungstiefe, als auch Agitation und Delir evaluiert und für neurointensive Patienten validiert ist (Sessler et al. 2002). Außerdem wurde gezeigt, dass es verlässliche Informationen bei der Beurteilung von SAB-Patienten liefert (Helbok et al. 2014; Riker et al. 2014).

Andere Methoden für die Bestimmung der Sedierungstiefe, wie der elektroenzephalographische Narcotrend®-Index oder der Bispectralindex (BIS), sind nicht für SAB validiert. Bei einer prospektiven Studie mit 35 Patienten mit TBI, zeigt BIS sich zuverlässiger für die Aufrechterhaltung einer stabilen Sedierung und vom ICP im Vergleich zu RASS (Yan et al. 2018). BIS wird in der Umfrage nach der Anwendung von RASS und ICP-Monitoring am dritthäufigsten verwendet, um die Sedierungstiefe zu bestimmen.

Moderne Überwachungssysteme zur Erfassung weiterer Parameter der zerebralen Perfusion und des Hirngewebes (Advanced/Multimodal monitoring), wie beispielsweise des $\mathrm{ptiO}_{2}$ und Überwachungsmessungen des Kreislaufs, wie beispielsweise die Messung des PulskonturHerzzeitvolumens (PiCCO) werden fast ausschließlich von UH und HVC angewendet. Es haben sowohl die American Heart Association guidelines for the management of $S A H$ (Connolly et al. 2012), als auch die Neurocritical Care Society Consensus (Diringer et al. 2011) empfohlen, modernes hämodynamisches Monitoring mit Thermodilutionsmethoden bei instabilen SAB-Patienten anzuwenden. 
Allerdings kann auch die Überwachung des $\mathrm{ptiO}_{2}$ bei schwergradigen SAB-Patienten wertvolle Informationen liefern und vor drohender DCI und Infarkten warnen (Sarrafzadeh et al. 2014). Zusätzlich empfiehlt die IMCCMM den Gebrauch von multimodalem Monitoring bei leichtgradiger SAB (Helbok et al. 2014, Bösel et al. 2013) (Tabelle 29). Multimodales Monitoring ist ein integrativer Ansatz, der verschiedene Überwachungsparameter wie beispielsweise Mikrodialyse, $\mathrm{ptiO}_{2}$ - Messungen, transkranielle Dopplersonographie und ICPund CPP-Messungen durch automatisierte Datenerfassung und computergestützte Techniken zusammenfasst (Dohmen und Sakowitz 2012). Der Verzicht, diese Überwachungssysteme anzuwenden, könnte einen nachteiligen Effekt auf das Outcome des Patienten haben. Es müssten allerdings mehr Daten erhoben werden, um zu evaluieren, welche Instrumente des Monitorings wirklich nützlich sind, da bislang das multimodale Neuromonitoring noch nicht ausreichend systematisch untersucht worden ist und diverse technische Herausforderungen darstellt (Schmidt et al. 2011).

Ein weiteres auffälliges Ergebnis der Umfrage ist die große Heterogenität bei den von den Zentren angegebenen Zielwerten des CPP. Die CPP-orientierte Therapie hat sich als wirksam bei TBI gezeigt, mit Empfehlung der Leitlinie einen CPP zwischen 60 - 70 mmHg aufrechtzuerhalten (Carney et al. 2017). Neueste Studien betonen die Wichtigkeit, den optimalen CPP (CPPopt) für jeden einzelnen Patienten mit TBI basierend auf deren individueller Autoregulation (bestimmt durch den pressure reactivity index (PRx, errechnet aus MAP und ICP)) individuell einzustellen (Aries et al. 2012; Kramer et al. 2019). Zwar wird der Einsatz dieses Index propagiert, allerdings liegt hierzu auch noch keine ausreichende Evidenz für die Therapiesteuerung und ICP-Kontrolle vor (Leitlinie Intrakranieller Druck 2018).

Zudem empfiehlt die S1-Leitlinie Intrakranieller Druck (DGN 2018), dass der CPP > 60 $\mathrm{mmHg}$ betragen sollte und bei der SAB bei ausgeprägten perfusionsrelevanten Vasospasmen $>80 \mathrm{mmHg}$. In der Umfragestudie geben die meisten Zentren einen leitliniengetreuen CPPopt von > $60 \mathrm{mmHg}$ bzw. $>70 \mathrm{mmHg}$, sowie eine fallabhängige Einstellung an. Insgesamt $27 \%$ aller Zentren bevorzugen eine CPPopt- Einstellung von $>80 \mathrm{mmHg}$. Ein zu niedriger CPP könnte das Entstehen von Ischämien und metobolischen Krisen begünstigen, denn ähnlich wie beim ICP existiert eine Assoziation eines niedrigen CPP mit schlechtem Outcome (Schmidt et al. 2011). Bei leichtgradiger SAB werden CPP-Werte $<70 \mathrm{mmHg}$ mit einer schlechten funktionellen Erholung und Hirngewebehypoxie assoziiert. Zusammengefasst zeigt sich der CPPopt als ein möglicherweise nützliches Instrument zur Diagnose und Behandlung der DCI (Bijlenga et al. 2010; Johnson et al. 2017). Es sind weitere Studien nötig, um die Wertigkeit des CPP beim Management der SAB zu definieren. 
Tabelle 29: Leitlinienempfehlungen zum Monitoring

\begin{tabular}{|c|c|c|}
\hline Referenz & Empfehlung & Patientenpopulation \\
\hline $\begin{array}{l}\text { AWMF } \\
\text { (Baron et al. 2015) }\end{array}$ & $\begin{array}{l}\text { Basierend auf den aktuellen Daten können keine } \\
\text { Empfehlungen für den Einsatz eines spezifischen } \\
\text { Instruments zur Überwachung von Analgesie } \\
\text { oder Sedierung bei Intensivpatienten mit schwe- } \\
\text { rer TBI und intrakranieller Hypertonie ausgespro- } \\
\text { chen werden. Neurologische Untersuchungen } \\
\text { sollten routinemäßig durchgeführt werden. }\end{array}$ & TBI \\
\hline $\begin{array}{l}\text { IMCCMM } \\
\text { (Bösel et al. 2013) }\end{array}$ & $\begin{array}{l}\text { Alle Patienten mit schwergradiger SAB sollten } \\
\text { überwacht und eine multimodale Überwachung in } \\
\text { Betracht gezogen werden. }\end{array}$ & SAB \\
\hline $\begin{array}{l}\text { Neurocritical Care } \\
\text { Society's Multidiscipli- } \\
\text { nary Consensus Con- } \\
\text { ference } \\
\text { (Diringer et al. 2011) }\end{array}$ & $\begin{array}{l}\text { Die Überwachung des Herzzeitvolumens kann } \\
\text { bei Patienten mit Anzeichen einer hämodynami- } \\
\text { schen Instabilität oder myokardialen Dysfunktion } \\
\text { nützlich sein. }\end{array}$ & $\mathrm{SAB}$ \\
\hline $\begin{array}{c}\text { AHA } \\
\text { (Connolly et al. 2012) }\end{array}$ & $\begin{array}{l}\text { Die Überwachung des Volumenstatus wird durch } \\
\text { die Überwachung des zentralen Venendrucks, } \\
\text { Lungenkegeldrucks und Flüssigkeitsbilanzierung, } \\
\text { ebenso wie die Behandlung mit kristallinen oder } \\
\text { kolloidalen Flüssigkeiten empfohlen. }\end{array}$ & $\mathrm{SAB}$ \\
\hline
\end{tabular}

\subsection{Ausblick}

Diese Umfrage spiegelt deutlich die Unterschiede des Patientenmanagements und der Neurointensivtherapie des Krankheitsbildes der SAB wider. Diese Heterogenität resultiert höchstwahrscheinlich aus dem großen Mangel und sich oftmals widersprechenden evidenzbasierten Daten, sowie deren unterschiedlicher Interpretation. Wie vorausgegangene Studien zeigen, kann die Heterogenität in der Behandlung zur Verschlechterung der Prognose der Patienten führen (Pronovost et al. 2008). In einem Kommentar von Rabinstein (2009) zu den Leitlinien von Bederson et al. (2009) werden wichtige Forschungsschwerpunkte für die nächsten Jahre herausgearbeitet, die sich mit Schlussfolgerungen dieser Arbeit überschneiden. Allerdings fokussieren sich diese mehr auf die Risikofaktoren zur Entstehung der SAB, die Diagnosestellung, den Aneurysmaverschluss, sowie auf das allgmeine Management von 
Komplikationen. Es wird nicht auf die Art der Sedierung sowie Medikamentenauswahl eingegangen, wie es in dieser Dissertationsschrift der Fall ist.

Die erhobenen Daten dieser Arbeit erlauben es folgende Themen zu identifizieren, welche in kontrolliert-randomisierte Studien zukünftig mit einbezogen werden könnten, um die VST bei SAB-Patienten zu standartisieren und somit zu verbessern:

Neben der Indikationsstellung zu Sedierung muss die Auswahl optimaler Sedativa für SABPatienten zur Einleitung, Aufrechterhaltung und Vertiefung der Sedierung festgelegt werden. Die Datenlage zu den optimalen Wirkungsprofilen bei der Anwendung bei SAB ist noch sehr gering. Die Umfragestudie zeigt eine große Vielfalt an Medikamenten und Dosierungen, sowie den weit verbreiteten Gebrauch von Propofol und Midazolam. Allerdings besteht keine eindeutige Empfehlung der Leitlinien zur Anwendung bei SAB (eine Anwendung wird in TBI-Leitlinien empfohlen (Baron et al. 2015)). Die pharmakologischen Eigenschaften der Sedativa können lebensbedrohliche Komplikationen der SAB auslösen, obwohl die Indikation zum Einsatz der Sedativa auch genau darin besteht, diese gefürchteten Komplikationen zu verhindern. Sedativa werden nicht nur für eine ICP- und zerebrale Stoffwechselkontrolle eingesetzt, sondern auch für die Reduktion von CSD, was zu DCI und schlechtem Outcome der Patienten beiträgt. So sind die Vor- und Nachteile der Sedierung, sowie alternative Medikamente, wie z. B. Ketamin weiter zu evaluieren.

Die Umfragestudie zeigt ein großes Potential für weitere Studien bei den Monitoringmöglichkeiten der Auswirkungen verschiedener Beatmungseinstellungen beim SAB-Patienten auf. Die Sedierung und maschinelle Beatmung des Patienten geht mit dem Verlust der klinischen Beurteilbarkeit des neurologischen Status einher. In der Umfragestudie werden unterschiedliche Indikationen zur Beatmung festgestellt (z. B. beim Horowitz-Index).

Hierbei ist eine Einheitlichkeit anzustreben. Desweiteren gibt es unterschiedliche Angaben zum Ausmaß der Hyperventilation, Normoventilation, Hyperoxie- und Hyperkapnie-Einstellungen. Diese Einstellungen werden hauptsächlich zur Prävention von Komplikationen bei SAB durchgeführt. Es zeigt sich deutlich wie sehr der Fokus in der Behandlung der SAB mit VST auf der Prävention der Komplikationen liegt. Zudem ist ein Ergebnis, dass für eine optimale Beatmungstherapie u. a. die Einstellung der $\mathrm{paCO}_{2}$ - und CPPopt-Werte in Abhängigkeit der patientenindividuellen Autoregulation erfolgen muss. Dies kann nur mit einem optimierten Monitoring der Patienten erfolgen, welches für die Zukunft eine große Herausforderung darstellt. 


\subsection{Limitationen der Studie}

Während die allgemeine Rücklaufquote mit 49\% ( $\mathrm{n}=50 / 103)$ relativ hoch war, wurden einige Fragen nur von einer Minderheit der teilnehmenden Zentren beantwortet. Das kann daran liegen, dass Fragen missverständlich formuliert waren oder übersehen wurden. Da nicht jede Frage beantwortet werden musste, bevor die nächste ausgefüllt werden konnte, könnte dies ein weiterer Faktor für die Nicht-Beantwortung einiger Fragen darstellen. Die Ergebnisse einer freiwilligen Umfrage haben zudem weder die Präzision noch die Aussagekraft einer wissenschaftlichen Studie. Dieser Umstand kann ebenfalls für die Rücklaufquote verantwortlich sein, die sonst höher gewesen sein könnte. Sicherlich wäre eine noch größere Zahl an Antworten für eine stärkere Aussagekraft dieser Arbeit förderlich. Auch die Form der Fragestellung trug zu unterschiedlichem Antwortverhalten mit bei und stellte eine große Schwierigkeit in der Auswertung und Vergleichbarkeit der Daten dar. Zudem sind sicherlich einfache Ankreuzfragen schneller beantwortet, als eine Frage im Freitextformat. Eine nicht zu unterschätzende Rolle spielt auch die Länge des Fragebogens mit 73 Fragen und der damit einhergehende Zeitaufwand von durchschnittlich ca. 20 bis 30 Minuten. Das ist viel Zeit für in der Klinik tätige Ärzte, die die Adressaten der Umfrage waren. Es haben sowohl Chefund Oberärzte als auch Assistenzärzte die Umfrage beantwortet. Daher sind die Ergebnisse mit Vorsicht zu interpretieren, da sie möglicherweise nicht vollständig repräsentativ für VSTPraktiken bei SAB-Patienten in Deutschland sind und stark von der Motivation und Genauigkeit der antwortenden Ärzte abhängen. Rückblickend kann zu dem Schluss gelangt werden, dass es sinnvoll gewesen wäre, schon in der frühsten Phase des Fragebogenentwurfs ein noch größeres interdisziplinäres Team zu involvieren, um noch fundiertere Fragen und Antwortmöglichkeiten zu erstellen. 


\section{$5 \quad$ Zusammenfassung}

In den letzten Jahrzehnten hat sich die Forschung zur intensivmedizinischen Therapie der $\mathrm{SAB}$, wie alle anderen klinischen Forschungsfelder auch, enorm weiterentwickelt. Gegenstand dieser Dissertation sind die Ergebnisse einer Umfrage zum Thema der intensivmedizinischen Therapie der SAB. Der Fragebogen bestand aus 73 Fragen zum intensivmedizinischen Management der SAB.

Die Umfragestudie hat eine hervorragende Repräsentativität und die Ergebnisse lassen eine gute Aussagekraft zu. Die herausgearbeiteten Unterschiede in der Behandlung spiegeln den generellen Mangel an qualitativ hochwertigen evidenzbasierten Daten wider. Zudem wird sehr deutlich, dass die vorhandenen Leitlinien im Hinblick auf die SAB Therapie durch die einzelnen Kliniken unterschiedlich interpretiert werden und dass der individuelle Spielraum zur Ausgestaltung der Therapiebestandteile an den 50 teilnehmenden Krankenhäuser sehr groß ist.

In einigen Zentren wurde die extrapolierte Anwendung von TBI-Leitlinien beim Management von SAB beobachtet. Beispielsweise wurden Schwellenwerte und Zieleinstellungen des CPPs aus der Leitlinie für die Behandlung der TBI übernommen, obwohl die Pathophysiologie beider Krankheitsbilder - TBI und SAB - völlig unterschiedlich ist. Klar wurde außerdem, dass eine Vielzahl verschiedener Sedativa in unterschiedlichsten Dosierungen eingesetzt werden. Desweiteren ergaben sich deutliche Unterschiede in der Indikation der mechanischen Ventilation der Krankenhäuser, was ein weiterer Hinweis auf den Mangel an Leitlinen zu diesem Thema darstellt.

Zudem war aus den Ergebnissen abzuleiten, dass UH und HVC im Vergleich zu LVC, mehr Übereinstimmung untereinander aufzeigten. Weitere Studien sind erforderlich, um die weitere Ausgestaltung und Feinheiten der VST im Rahmen von SAB zu definieren. Da die Prognose eines SAB-Patienten von einer Vielzahl von Faktoren abhängt, kann die Etablierung evidenzbasierter Protokolle für das Management dieser Patienten auf der Intensivstation zu einer Optimierung des Behandlungsmanagements und zu verbesserten Ergebnissen hinsichtlich der Prognose führen.

Die vorliegende Dissertationsschrift zeigt, wie wichtig es ist, die Behandlungsmethoden dem aktuellen Stand der Wissenschaft anzupassen. Es wird deutlich, dass die Einstellungen und Praktiken in Bezug auf die VST bei SAB sehr heterogen sind. 
6 Anhang

\title{
Umfragestudie zum Sedierungsmanagement von Subarachnoidalblutungen (SAB)
}

Vielen Dank für Ihre Teilnahme!

\author{
Allgemeines \\ 1* Name des teilnehmenden Zentrums
}

2 Wie viele Betten hat Ihr Krankenhaus? (Zahl)

3 Aus welcher Stadt nehmen Sie teil?

4* Name des Ansprechpartners

5* Wie wird die Intensivstation geleitet?

rein neurochirurgisch

$\square$ rein anästhesiologisch

gemischt

neurochirurgisch/neurologische

Mitarbeit

Andere Antwort 
6 Wie häufig am Tag ist Oberarztvisite?

$\square_{>3 \mathrm{mal}} 1 \mathrm{mal} 2 \mathrm{mal} \quad \square$ mal

Andere Antwort

(n)

7 In welchem Schichtmodell arbeiten Sie?
24h Dienst
Dreischichtmodell
$12 \mathrm{~h}$ Modell

Andere Antwort

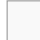

8 Wie ist Ihr Belegschlüssel? (Facharzt/Assistenzarzt)

$\square$ 1:4 $1: 2 \quad \square 2: 3$

Andere Antwor

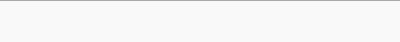

* Wie häufig finden interdisziplinäre Visiten mit z.B. Internisten, Mikrobiologen, etc. statt?

$\square_{\text {nie }}$ täglich mehrfach wöchentlich $\square$ einmal pro Woche

10 Wie ist Ihr Behandlungsvolumen im Hinblick auf die aneurysmatische Subarachnoidalblutung zusammengefasst auf Allgemeinstationen, Intermediate Care Stationen und Intensivstationen (n/Jahr)?

11* Wie viele Patienten mit SAB behandeln Sie in Ihrem Zentrum geschätzt prozentual auf der Intensivstation / Jahr (Anzahl/ Jahr)?

Vielen Dank! Nun folgt der spezifische Teil. 


\section{Intensivmedizinische Behandlung von aneurysmatischer Subarachnoidalblutung}

Management von Beatmung

12 Wie viele Ihrer SAB Patienten sind intubiert/beatmet und analgosediert (\%)?

\begin{tabular}{|c|c|c|}
\hline$<10 \%$ & $10-20 \%$ & $20-30 \%$ \\
\hline $30-40 \%$ & $40-50 \%$ & $<50 \%$ \\
\hline $50-60 \%$ & $70-80 \%$ & $>80 \%$ \\
\hline
\end{tabular}

13* Gibt es bei Ihnen eine Indikation zur maschinellen Beatmung bei SAB modified Fisher 3 oder SAB mit begleitender ICB/VEB (modified Fisher 4)?
$\mathrm{Ja}$
$\int$ Nein

Andere Antwort

14 Bei Indikation zur maschinellen Beatmung aufgrund der reduzierten Vigilanz/WFNS Grad (World Federation of Neurosurgical Societies) - ab welchem Schwellenwert wird das bei Ihnen regelmäßig / standardisiert durchgeführt?

$\square \operatorname{Grad} 1$
$\square \operatorname{Grad} 4$

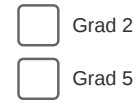

$\square \operatorname{Grad} 3$
$\square$ WFNS Grad keine formale
Indikation

15 Bei Indikation zur maschinellen Beatmung: Pulmonaler Gasaustausch, Aspiration: Wird bei Innen der Horowitz Quotient in die Bewertung der Indikation zur Beatmung miteinbezogen? (Horowitz Index = arterieller Sauerstoffpartialdruck (paO2)/ O2 Fraktion der Atemluft)

$\mathrm{Ja}$

$\square$ Nein 
16 Indikation zur maschinellen Beatmung: Pulmonaler Gasaustausch, Aspiration:

Ab welchem Horowitz Quotient wird bei Ihnen die Indikation zur maschinellen invasiven Beatmung gestellt?

(Horowitz Index = arterieller Sauerstoffpartialdruck (paO2) / O2 Fraktion der Atemluft)

Horowitz Index $<200 \quad \square$ Horowitz Index $<100$

17 Indikation zur maschinellen Beatmung:

Ist ein cerebraler Vasospasmus, welcher in der initialen Angiographie diagnostiziert wird, bei Ihnen ein Grund für eine maschinelle Beatmung/ Sedierungsperiode?

ja

nein

18 Indikation zur maschinellen Beatmung:

Stellen Sie eine Indikation zur maschinellen Beatmung und Analgosedierung, falls die SAB durch eine signifikanten kardiale Mitbeteiligung verkompliziert wird?

$\square$ ja

$\square$ nein

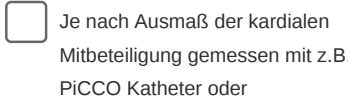

Picco Katheter oder

Echokardiographie

19 Wenn die Indikation zur maschinellen Beatmung gestellt wird - wie hoch ist der angestrebte paO2 $(\mathrm{mmHg})$ ?

Steuerung nach $\mathrm{SaO} 2$

$80-100$

$>100$

20 Wenn die Indikation zu einer maschinellen Beatmung gestellt wird - wie hoch ist der angestrebte paCO2 $(\mathrm{mmHg})$ ?

$<35$

$35-45$

$\int>45$

21 Sonstige Indikationen zur maschinellen Beatmung bei SAB Patienten: 
22 Gibt es in Ihrer Klinik einen Optimum paO2, auf den die Beatmungseinstellungen angepasst werden?

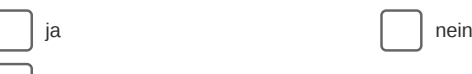

Falls ja, wie ist der Wert?

rat

23 Gibt es in Ihrer Klinik einen Optimum paCO2, auf den die Beatmungseinstellungen angepasst werden?

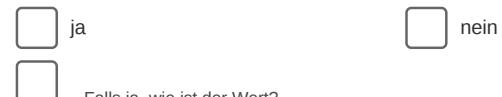

alls ja, wie ist der Wert?

24 Welches kontrollierte maschinelle Beatmungsverfahren ist auf Ihrer Intensivstation das Standardbeatmungsverfahren bei sedierten Patienten?

Druckkontrollierte Beatmung mit Möglichkeit der Spontanatmung

auf oberem und unterem Beatmung

Druckniveau / BIPAP (Biphasic

Positive Airways Pressure) 


\section{Intensivmedizinische Behandlung von aneurysmatischer Subarachnoidalblutung}

Management von Analgosedierung

25* Welche Kombination aus Medikamenten ist Mittel der Wahl zur Induktion/ Einleitung von Sedierung bei SAB Patienten? (Mehrfachauswahl möglich)
$\square$ Ketamin
$\square$ Benzodiazepin
Propofol
Immer Kombination mit Opiat
Immer Kombination mit
Muskelrelaxans

Andere Antwort

26 Wird ein Benzodiazepin zur Aufrechterhaltung verwendet?
$\square$ Nein
$\mathrm{Ja}$
$\square$ Midazolam
Diazepam

Andere Antwort

27 Welche Kombination aus i.v. Sedativa wird in Ihrer Klinik standardisiert bei Indikation zur Aufrechterhaltung der Analgosedierung zuerst verabreicht?

28

Wird ein Opioid verwendet?

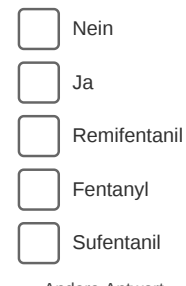

Andere Antwort 
29 Welche Dosierung des Benzodiazepins ist die maximale Dosierung pro Stunde bei einem normgewichtigen Erwachsenen? (mg/h)

30 Welche Dosierung des Opiats ist die maximale Dosierung pro Stunde bei einem normgewichtigen Erwachsenen? $(\mu \mathrm{g}(\mathrm{mg}) / \mathrm{h})$

31 Welches Verfahren zur Objektivierung von Sedierungstiefe verwenden Sie?

$\square$ Bispectralindex $\square$ RASS Score $\square$ Narco Trend
$\square$ ICP

Anderes Verfahren

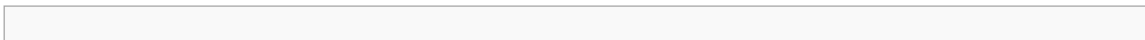

32 Monitoring der Sedierungstiefe: wie wird in Ihrer Klinik der intrakranielle Druck (ICP) gemessen?

$\square$ ICP wird nicht gemessen $\square$ über ICP Sonde $\square$ über Externe Ventrikeldrainage
andere druckregulierte Drainagesysteme wie zB Liquogard

33 Verwenden Sie in Ihrer Klinik ein CPP gesteuertes Blutdruckmanagement in der Intensivtherapie bei SAB Patienten?

$\square$ ja $\longrightarrow$ nein

34 Welches ist inr "Optimum Cerebral Perfusion Pressure (CPP)" bei SAB Behandlung? 


\section{Management von Analgosedierung}

Erweitertes intensivmedizinisches Monitoring_Erweiterung der Sedierung

35 Gibt es eine regelhafte Pulskonturanalyse und Thermodilution (PiCCO Monitoring),

Pulmonalarterienkatheter oder intermittierende Echokardiographie bei den sedierten SAB Patienten?

ja

nein

36* Findet in der Standardbehandlung von intubiert beatmeten, analgosedierten SAB Patienten in Ihrer Klinik regelmäßig eine Messung des cerebralen Sauerstoffpartialdrucks (ptiO2) statt?

ja

nein

37 Wie wird nach dem Ausschluss einer behandelbaren ICP Erhöhung (z.B. Liquordrainage erhöhen, intracerebrales Hämatom entfernen, zusätzliche EVD (Externe Ventrikeldrainage), Anpassung der Lagerung, Anpassung der Beatmung/ Normocapnie, Antiepileptika, Normothermie, etc.) die Diagnose einer möglicherweise insuffizienten Sedierung gestellt?

klinisch

Anhand von

anhand des ICP

elektrophysiologischem Monitoring

(BIS/Narcotrend)

Andere Antwort

Vertiefung der Sedierung

38* Welches Medikament ist bei SAB Patienten in Ihrer Klinik das Mittel der Wahl zur Vertiefung der Sedierung?

$\begin{array}{lll}\square \text { Gammahydroxybutyrat } & \square \text { Clonidin } & \square \text { anderes Benzodiazepin } \\ \square \text { Ketamin } & \square \text { Propofol }\end{array}$

Andere Antwort 
39 Wie viele Sedativa werden maximal eingesetzt? (Anzahl)
1
$\int 2$
$>3$

Andere Antwort

Ande

40* Welche weiteren Medikamente zur Vertiefung der Analgosedativa werden regelmäßig eingesetzt?

$\begin{array}{ll}\square \text { Gammahydroxybutyrat } & \square \text { Clonidin } \\ \square_{\text {Andere Antwort }} \text { Propofol } & \square \text { anderes Opiat }\end{array}$

Andere Antwort

41 Wenn Propofol: Welche Dosierung des Propofols ist die maximale Dosierung pro Stunde bei einem normgewichtigen Erwachsenen? (mg/h)

42 Wie oft sehen Sie Komplikationen im Zusammenhang mit Propofolgabe?

sehr selten $(<1 \%)$

selten (zwischen 1 - $10 \%)$

häufig $(>10 \%)$

43 Welche Komplikationen mit Propofol treten auf?

44 Wie lange wird Propofol gegeben?

maximal 3 Tage

maximal 7 Tage

länger als 7 Tage

45 Wenn Ketamin: Welche Dosierung des Ketamins ist die maximale Dosierung pro Stunde bei einem normgewichtigen Erwachsenen? (mg/h) 
46 Wenn Ketamin: verwenden Sie bei Ketamin Sedierung regelhaft ein erweitertes kardiales Monitoring ((Pulskonturanalyse und Thermodilution (PiCCO Monitoring), Pulmonalarterienkatheter oder intermittierende Echokardiographie) z.B. aufgrund der bekannten negativen Inotropie...) ja

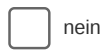

47 Werden bei Ihnen zur Vertiefung der Sedierung Barbiturate eingesetzt?
ja
nein

48 Wie und wann werden Barbiturate eingesetzt?

kurzzeitig

$\square$ langzeitig

kein Einsatz

49 Wird bei Ihnen inhalative Sedierung (z.B. mit dem AnaConDa Device) benutzt?

ja $\square$ nein

Es ist mir nicht bekannt, dass

inhalative Sedierung als

Möglichkeit zur Sedierung bei SAB

Patienten eingesetzt werden kann

50 Wie sind Ihre Erfahrungen mit inhalativer Sedierung bei SAB Patienten?
Überwiegend gut
Überwiegend Schlecht
Risikoreiches Verfahren
keine Erfahrung
Andere Antwort 


\section{Intensivmedizinisches Management bei SAB Patienten}

Fragen zu anderen interventionellen Maßnahmen und zum Auftreten von Komplikationen

51 Was gilt als hausinterne Schwelle zur dekompressiven Hemicraniektomie bei SAB Patienten?

52 Wann wird in Ihrer Klinik ein langzeitbeatmeter SAB Patient tracheostomiert?

früh (<3 Tage)

zwischen 3 - 10 Tag

eher spät (> 10 Tage)

Reduktion der Analgosedierung, Komplikationen

und mögliche Nebenwirkungen

53 In welchen Reduktionsschritten wird bei Ihnen die Sedierung reduziert? ( \% der Höchstdosis)

54 Was wird als leichte Sedativa-assoziierte Nebenwirkung/Komplikation wahrgenommen?

$\square$ leichte Obstipationen
Tiefe Beinvenenthrombose
milde Erhöhung der
Cransaminasen
Critical-Iness-Polyneuropathie


55 Was wird als schwere Sedativa-assoziierte Nebenwirkung/Komplikation wahrgenommen?

$\square$ leichte Obstipationen
Eiefe Beinvenenthrombose
Erhöhung der Transaminasen
Dekubitical-Inness-Polyneuropathie

56 Sedativa-assoziierte Komplikationen sehen wir bei SAB Patienten in unserer Klinik...
sehr selten $(<1 \%)$
selten $(1-10 \%)$
häufig $(>10 \%)$

57 Wie viele ventilatorassoziierte Pneumonien gab es bei Ihnen bei den letzten 30 analgosedierten SAB Patienten (alternativ bei den intubiert beatmeten, analgosedierten SAB Patienten im Jahr 2016)? (Anzahl)
$\square<10$
10- 20
21-30
$31-40$

Andere Antwort

58 Kommt bei Ihnen extensivierte medikamentöse Stimulation der Magen / Darm Passage aufgrund von Sedativa - induzierter Darmmotilitätsstörung zum Einsatz?
Ja, immer
Ja, bei gestörter Darmmotilität
$\mathrm{Nie}$

Andere Antwor 
59 Wie viele Laparotomien (z.B. aufgrund von lleus - Problematik oder Obstipation) gab es bei Ihnen bei den letzten 30 analgosedierten SAB Patienten ? (Anzahl)

(alternativ bei den intubiert beatmeten, analgosedierten SAB Patienten im Jahr 2016 - dann bitte Antwort in Klammern setzen)
wenige $<5$
5-10
$>10$

Andere Antwor

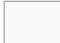

60 Wie viele sedierungsassoziierte Lungenembolien gab es bei Ihnen bei den letzten 30 analgosedierten SAB Patienten? (Anzahl)

(alternativ bei den intubiert beatmeten, analgosedierten SAB Patienten im Jahr 2016 - dann bitte Antwort in Klammern setzen)
$<5$ von 30
$\square>5$ von 30
$>10$ von 30

Andere Antwort

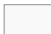

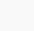

61 Wie viele sedierungsassoziierte Transaminase-Erhöhungen/Cholestaseparameter - Erhöhungen etc. gab es bei Ihnen bei den letzten 30 analgosedierten SAB Patienten? (Anzahl)

(alternativ bei den intubiert beatmeten, analgosedierten SAB Patienten im Jahr 2016 - dann bitte Antwort in Klammern setzen)
$<5$ von 30
$>5$ von 30
$>10$ von 30

Andere Antwor

(20)

62 Wie häufig sehen Sie in Ihrer Klinik hämodynamische Komplikationen von Sedierungstherapie wie z.B. einen zügigen Blutdruckabfall? (\% der intubiert - beatmeten SAB Patienten)
$<10 \%$
$10-50 \%$
$>50 \%$

Andere Antwort

63 Können Sie sich an eine drastische Komplikation erinnern, die durch eine zu tiefe Sedierung hervorgerufen worden ist?

$\square$ ja nein 
64 Um was für eine Komplikation handelte es sich?

65 Mit welchem Medikament war diese assoziiert?

66 Wie lange liegt diese zurück? (Monate)

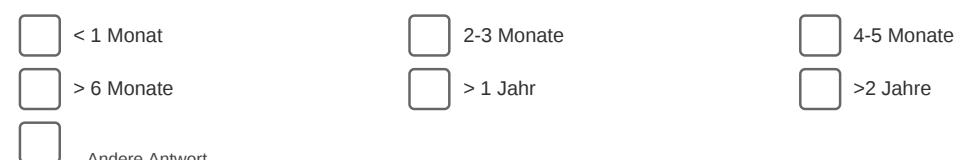

Andere Antwort

67 Hätte man diese Komplikation vermeiden können? Wenn ja, wodurch?

68 Können Sie sich an eine drastische Komplikation erinnern, die durch eine zu lange Sedierung hervorgerufen worden ist?
$\int \mathrm{ja}$
$\int$ nein

69 Um was für eine Komplikation handelte es sich?

70 Mit welchem Medikament war diese assoziiert? 
71 Wie lange liegt diese zurück? (Monate)
$\square<1$ Monat
2-3 Monate
4-5 Monate
$\square>6$ Monate
$\square>1$ Jahr
>2 Jahre
Andere Antwort

72 Hätte man diese Komplikation vermeiden können? Wenn ja, wodurch?

73 Wie beenden Sie die Sedierung?

Vielen Dank für Ihre Teilnahme an dieser Studie! 


\section{$7 \quad$ Literaturverzeichnis}

Adembri C, Venturi L, Pellegrini-Giampietro DE (2007): Neuroprotective effects of propofol in acute cerebral injury. CNS Drug Rev $\underline{13}$, 333-351

Alotaibi NM, Wang JZ, Pasarikovski CR, Guha D, Al-Mufti F, Mamdani M, Saposnik G, Schweizer TA, Macdonald RL (2017): Management of raised intracranial pressure in aneurysmal subarachnoid hemorrhage: time for a consensus? Neurosurg Focus $\underline{43}$, E13

Aries MJ, Czosnyka M, Budohoski K, Steiner L, Lavinio A, Kolias A, Hutchinson P, Brady K, Menon D, Pickard J et al. (2012): Continuous determination of optimal cerebral perfusion pressure in traumatic brain injury. Crit Care Med $\underline{40}$, 2456-2463

Baron R, Binder A, Biniek R, Braune S, Buerkle H, Dall P, Demirakca S, Eckardt R, Eggers V, Eichler I et al. (2015): Evidence and consensus based guideline for the management of delirium, analgesia, and sedation in intensive care medicine. Revision 2015 (DAS-Guideline 2015) short version. Ger Med Sci $\underline{13}$, Doc19

Barr J, Fraser GL, Puntillo K, Ely EW, Gélinas C, Dasta JF, Davidson JE, Devlin JW, Kress JP, Joffe AM et al. (2013): Clinical practice guidelines for the management of pain, agitation, and delirium in adult patients in the intensive care unit: Executive summary. Am J Health Syst Pharm $\underline{70}, 53-58$

Bauer T, Ritz R, Haberthür C, Haefeli W, Scollo-Lavizzari G, Ha H, Hunkeler W, Sleight A (1995): Prolonged sedation due to accumulation of conjugated metabolites of midazolam. The Lancet $\underline{346}, 145-147$

Bederson J, Connolly S, Batjer H, Dacey R, Dion J, Diringer M, Duldner J, Harbaugh R, Patel A, Rosenwasser R (2009): Guidelines for the management of aneurysmal subarachnoid hemorrhage. Stroke $\underline{40}, 994-1025$

Bijlenga P, Czosnyka M, Budohoski KP, Soehle M, Pickard JD, Kirkpatrick PJ, Smielewski P (2010): "Optimal cerebral perfusion pressure" in poor grade patients after subarachnoid hemorrhage. Neurocrit Care 13, 17-23

Bösel J, Schiller P, Hook Y, Andes M, Neumann J, Poli S, Amiri H, Schönenberger S, Peng Z, Unterberg A et al. (2013): Stroke-related early tracheostomy versus prolonged orotracheal intubation in Neurocritical Care Trial (SETPOINT). Stroke $\underline{44}, 21-28$

Bratton SL, Chestnut RM, Ghajar J, McConnell F, Harris OA, Hartl R, Manley GT, Nemecek A, Newell DW, Rosenthal G et al. (2007): XI. Anesthetics, Analgesics, and Sedatives. J Neurotrauma $\underline{24}, 71$

Caeiro L, Menger C, Ferro JM, Albuquerque R, Figueira ML (2005): Delirium in acute subarachnoid haemorrhage. Cerebrovasc Dis 19 , 31-38

Carlson AP, Abbas M, Alunday RL, Qeadan F, Shuttleworth CW (2018): Spreading depolarization in acute brain injury inhibited by ketamine: a prospective, randomized, multiple crossover trial. J Neurosurg 130, 1513-1519 
Carney N, Totten AM, O’Reilly C, Ullman JS, Hawryluk GWJ, Bell MJ, Bratton SL, Chesnut R, Harris OA, Kissoon N et al. (2017): Guidelines for the management of severe traumatic brain injury, Fourth Edition. Neurosurgery $\underline{80}$, 6-15

Chan KH, Dearden NM, Miller JD, Andrews PJ, Midgley S (1993): Multimodality monitoring as a guide to treatment of intracranial hypertension after severe brain injury. Neurosurgery $\underline{32}$, $547-552$

Cho WS, Kim JE, Park SQ, Ko JK, Kim DW, Park JC, Yeon JY, Chung SY, Chung J, Joo SP et al. (2018): Korean clinical practice guidelines for aneurysmal subarachnoid hemorrhage. J Korean Neurosurg Soc $\underline{61}, 127$

Citerio G, Cormio M (2003): Sedation in neurointensive care: advances in understanding and practice. Curr Opin Crit Care 9, 120-126

Claassen J, Vu A, Kreiter KT, Kowalski RG, Du EY, Ostapkovich N, Fitzsimmons B-FM, Connolly ES, Mayer SA (2004): Effect of acute physiologic derangements on outcome after subarachnoid hemorrhage. Crit Care Med 32, 832-838

Committee for Guidelines for Management of Aneurysmal Subarachnoid Hemorrhage, Japanese Society on Surgery for Cerebral Stroke (2012): Evidence-Based Guidelines for the Management of Aneurysmal Subarachnoid Hemorrhage English Edition. Neurol Med Chir (Tokyo) $\underline{52}, 355-429$

Connolly E, Rabinstein A, Carhuapoma R, Derdeyn C, Dion J, Higashida RT., Hoh BL., Kirkness CJ, Naidech AM, Ogilvy CS et al. (2012): Guidelines for the management of aneurysmal subarachnoid hemorrhage. Stroke $\underline{43}, 1711-1737$

Coppadoro A, Citerio G (2011): Subarachnoid hemorrhage: an update for the intensivist. Minerva Anestesiol 7ㅜ, 74-84

Cross DT, Tirschwell DL, Clark MA, Tuden D, Derdeyn CP, Moran CJ, Dacey RG (2003): Mortality rates after subarachnoid hemorrhage: variations according to hospital case volume in 18 states. J Neurosurg $\underline{99}$, 810-817

Curley G, Kavanagh BP, Laffey JG (2010): Hypocapnia and the injured brain: more harm than benefit. Crit Care Med $\underline{38}, 1348-1359$

Dankbaar JW, Slooter AJ, Rinkel GJ, Schaaf IC (2010): Effect of different components of triple-H therapy on cerebral perfusion in patients with aneurysmal subarachnoid haemorrhage: a systematic review. Crit Care 14, R23

de Gans K, Nieuwkamp DJ, Rinkel GJE, Algra A (2002): Timing of aneurysm surgery in subarachnoid hemorrhage: a systematic review of the literature. Neurosurgery $\underline{50}, 336-340$

Dereeper E, Berré J, Vandesteene A, Lefranc F, Vincent J-L (2002): Barbiturate coma for intracranial hypertension: Clinical observations. J Crit Care 17, 58-62

Devabhakthuni S, Armahizer MJ, Dasta JF, Kane-Gill SL (2012): Analgosedation: A paradigm shift in intensive care unit sedation practice. Ann Pharmacother 46, 530-540 
DGN (2018): Intrakranieller Druck (Leitlinien für Diagnostik und Therapie in der Neurologie). S1Leitlinie der Deutschen Gesellschaft für Neurologie. https://www.dgn.org/images/red_leitlinien/LL_2018/PDFs_Download/030105_LL_Intrakranieller_Druck_2018.pdf, abgerufen am 11.11.2019

DGN (2012): Subarachnoidalblutung (Leitlinien für Diagnostik und Therapie in der Neurologie). S1-Leitlinie der Deutschen Gesellschaft für Neurologie. https://www.dgn.org/leitlinien/231811-26-2012-subarachnoidalsblutung-sab; abgerufen am 13.10.2019

Diringer MN, Bleck TP, Hemphill JC, Menon D, Shutter L, Vespa P, Bruder N, Connolly ES, Citerio G, Gress D et al. (2011): Critical care management of patients following aneurysmal subarachnoid hemorrhage: recommendations from the Neurocritical Care Society's Multidisciplinary Consensus Conference. Neurocrit Care 15, 211

Dohmen C, Sakowitz OW (2012): Multimodales Monitoring in der Neurointensivmedizin. Nervenarzt $\underline{83}, 1559-1568$

Dorsch NWC (2002): Therapeutic approaches to vasospasm in subarachnoid hemorrhage. Curr Opin Crit Care $\underline{8}, 128-133$

Doukas A, Barth H, Petridis KA, Mehdorn M, von der Brelie C (2019): Misdiagnosis of acute subarachnoid hemorrhage in the era of multimodal diagnostic options. Am J Emerg Med 37, 2079-2083

Dreier JP (2011): The role of spreading depression, spreading depolarization and spreading ischemia in neurological disease. Nat Med 17, 439-447

Dupont SA, Wijdicks EFM, Lanzino G, Rabinstein AA (2010): Aneurysmal subarachnoid hemorrhage: An overview for the practicing neurologist. Semin Neurol $\underline{30}, 545-554$

Egerod I (2009): Cultural changes in ICU sedation management. Qual Health Res 19, 687-696

Ely EW, Shintani A, Truman B, Speroff T, Gordon SM, Frank E. Harrell J, Inouye SK, Bernard GR, Dittus RS (2004): Delirium as a predictor of mortality in mechanically ventilated patients in the intensive care unit. JAMA 291, 1753-1762

Feigin VL, Rinkel GJ, Algra A, Vermeulen M, van Gijn J (1998): Calcium antagonists in patients with aneurysmal subarachnoid hemorrhage: a systematic review. Neurology $\underline{50}, 876-883$

Feigin VL, Rinkel GJ, Algra A, van Gijn J (2000): Circulatory volume expansion for aneurysmal subarachnoid hemorrhage. Cochrane Database Syst Rev CD000483

Feigin VL, Lawes CM, Bennett DA, Barker-Collo SL, Parag V (2009): Worldwide stroke incidence and early case fatality reported in 56 population-based studies: a systematic review. Lancet Neurol $\underline{8}, 355-369$

Finfer SR, Ferch R, Morgan MK (1999): Barbiturate coma for severe, refractory vasospasm following subarachnoid haemorrhage. Intensive Care Med 25, 406-409

Firsching R, Rickels E, Mauer UM, Sakowitz OW, Messing-Jünger M et al. (2015): Leitlinie SchädelHirn-Trauma im Erwachsenenalter. DGNC. AWMF 008/001

Fisher CM, Kistler JP, Davis JM (1980): Relation of cerebral vasospasm to subarachnoid hemorrhage visualized by computerized tomographic scanning. Neurosurgery $\underline{6}, 1-9$ 
Francoeur CL, Mayer SA (2016): Management of delayed cerebral ischemia after subarachnoid hemorrhage. Crit Care 20, 277

Friedman J, Pichelmann M, Piepgras D, McIver J, Toussaint L, McClelland RL, Nichols D, Meyer F, Atkinson J, Wijdicks E (2003): Pulmonary complications of aneurysmal subarachnoid hemorrhage. Neurosurgery $\underline{52}, 1025-1032$

Helbok R, Kurtz P, Schmidt MJ, Stuart MR, Fernandez L, Connolly SE, Lee K, Schmutzhard E, Mayer SA, Claassen J, Badjatia N (2012): Effects of the neurological wake-up test on clinical examination, intracranial pressure, brain metabolism and brain tissue oxygenation in severely brain-injured patients. Crit Care 16, R226

Helbok R, Olson DM, Roux PDL, Vespa P (2014): Intracranial pressure and cerebral perfusion pressure monitoring in non-TBI patients: Special Considerations. Neurocrit Care 21, 85-94

Heo DH, Hu C, Cho SM, Whang K, Pyen JS, Kim HJ (2003): Barbiturate coma therapy in severe and refractory vasospasm following subarachnoid hemorrhage. J Korean Neurosurg Soc $\underline{33}$, $142-148$

Hernández-Durán S, Salfelder C, Schaeper J, Moerer O, Rhode V, Mielke D, von der Brelie C (2020): Mechanical ventilation, sedation and neuromonitoring of patients with aneurysmal subarachnoid hemorrhage in Germany: Results of a nationwide survey. Neurocrit Care 34, 236247

Hertle DN, Dreier JP, Woitzik J, Hartings JA, Bullock R, Okonkwo DO, Shutter LA, Vidgeon S, Strong AJ, Kowoll C et al. (2012): Effect of analgesics and sedatives on the occurrence of spreading depolarizations accompanying acute brain injury. Brain 135, 2390-2398

Hop JW, Rinkel GJ, Algra A, van Gijn J (1997): Case-fatality rates and functional outcome after subarachnoid hemorrhage. Stroke $\underline{28}, 660-664$

Hunt WE, Hess RM (1968): Surgical risk as related to time of intervention in the repair of intracranial aneurysms. J Neurosurg $\underline{28}, 14-20$

Jackson JC, Gordon SM, Hart RP, Hopkins RO, Ely EW (2004): The association between delirium and cognitive decline: A review of the empirical literature. Neuropsychol Rev $\underline{14}, 87-98$

Jamadarkhana S, Gopal S (2010): Clonidine in adults as a sedative agent in the Intensive Care Unit. J Anaesthesiol Clin Pharmacol 26, 439-445

Johnson U, Engquist H, Lewén A, Howells T, Nilsson P, Ronne-Engström E, Rostami E, Enblad P (2017): Increased risk of critical CBF levels in SAH patients with actual CPP below calculated optimal CPP. Acta Neurochir (Wien) 159, 1065-1071

Kahn JM, Caldwell EC, Deem S, Newell DW, Heckbert SR, Rubenfeld GD (2006): Acute lung injury in patients with subarachnoid hemorrhage: incidence, risk factors, and outcome. Crit Care Med $\underline{34}, 196-202$

Kim YI, Park SW, Nam TK, Park YS, Min BK, Hwang SN (2008): The effect of barbiturate coma therapy for the patients with severe intracranial hypertension: A 10-year experience. J Korean Neurosurg Soc 44, 141-145 
Kramer AH, Couillard PL, Zygun DA, Aries MJ, Gallagher CN (2019): Continuous assessment of "optimal" cerebral perfusion pressure in traumatic brain injury: A cohort study of feasibility, reliability, and relation to outcome. Neurocrit Care 30, 51-61

Leitlinie Intrakranieller Druck: siehe DGN (2018).

Leitlinie Subarachnoidalblutung: siehe DGN (2012).

Linn F, Voorbij H, Rinkel GJE, Algra A, van Gijn J (2005): Visual inspection versus spectrophotometry in detecting bilirubin in cerebrospinal fluid. J Neurol Neurosurg Psychiatry $\underline{76}, 1452-$ 1454

Macdonald RL (2014): Delayed neurological deterioration after subarachnoid haemorrhage. Nat Rev Neurol 10, 44-58

Manoel A, Goffi A, Marotta TR, Schweizer TA, Abrahamson S, Macdonald RL (2016): The critical care management of poor-grade subarachnoid haemorrhage. Crit Care 20, 21

Marion DW, Puccio A, Wisniewski SR, Kochanek P, Dixon CE, Bullian L, Carlier P (2002): Effect of hyperventilation on extracellular concentrations of glutamate, lactate, pyruvate, and local cerebral blood flow in patients with severe traumatic brain injury. Crit Care Med 30, 26192625

Mattia C, Savoia G, Paoletti F, Piazza O, Albanese D, Amantea B, Ambrosio F, Belfiore B, Berti M, Bertini L et al. (2006): SIAARTI Recommendations for analgo-sedation in intensive care unit. Minerva Anestesiol 72,37

McNeill L, English S, Borg N, Matta B, Menon D (2013): Effects of institutional caseload of subarachnoid hemorrhage on mortality. Stroke $\underline{44}, 647-652$

Mees SD, Rinkel GJ, Feigin VL, Algra A, Bergh WM van den, Vermeulen M, Gijn J van (2007): Calcium antagonists for aneurysmal subarachnoid haemorrhage. Cochrane Database Syst Rev, CD000277

Meixensberger J, Kunze E, Barcsay E, Vaeth A, Roosen K (2001): Clinical cerebral microdialysis: brain metabolism and brain tissue oxygenation after acute brain injury. Neurol Res $\underline{23}$, 801-806

Meurer WJ, Walsh B, Vilke GM, Coyne CJ (2016): Clinical guidelines for the emergency department evaluation of subarachnoid hemorrhage. J Emerg Med 50, 696-701

Mielke D, Malinova V, Moerer O, Suntheim P, Voit M, Rohde V (2019): Does the subspecialty of an intensive care unit (ICU) has an impact on outcome in patients suffering from aneurysmal subarachnoid hemorrhage? Neurosurg Rev 42, 147-153

Molyneux A, Kerr R, Stratton I, Sandercock P, Clarke M, Shrimpton J, Holman R, International Subarachnoid Aneurysm Trial (ISAT) Collaborative Group (2002): International Subarachnoid Aneurysm Trial (ISAT) of neurosurgical clipping versus endovascular coiling in 2143 patients with ruptured intracranial aneurysms: a randomised trial. The Lancet $\underline{360}, 1267-1274$

Nassar AP, Park M (2016): Sedation protocols versus daily sedation interruption: a systematic review and meta-analysis. Rev Bras Ter Intensiva $\underline{28}$, 444-451 
Nelsen JL, Haas CE, Habtemariam B, Kaufman DC, Partridge A, Welle S, Forrest A (2008): A prospective evaluation of propylene glycol clearance and accumulation during continuous-infusion lorazepam in critically ill patients. J Intensive Care Med $\underline{23}$, 184-194

Nuño M, Patil CG, Lyden P, Drazin D (2012): The effect of transfer and hospital volume in subarachnoid hemorrhage patients. Neurocrit Care 17, 312-323

Oddo M, Crippa IA, Mehta S, Menon D, Payen J-F, Taccone FS, Citerio G (2016): Optimizing sedation in patients with acute brain injury. Crit Care 20, 128

Paolone S (2017): Extracorporeal Membrane Oxygenation (ECMO) for Lung Injury in Severe Acute Respiratory Distress Syndrome (ARDS): Review of the literature. Clin Nurs Res $\underline{26}$, 747-762

Patel B, Chatterjee S, Davignon S, Herlihy JP (2019): Extracorporeal membrane oxygenation as rescue therapy for severe hypoxemic respiratory failure. J Thorac Dis $\underline{11}$, 1688-1697

Petridis AK, Doukas A, Kienke S, Maslehaty H, Mahvash M, Barth H, Mehdorn HM (2010): The effect of lung-protective permissive hypercapnia in intracerebral pressure in patients with subarachnoid haemorrhage and ARDS. A retrospective study. Acta Neurochir (Wien) 152, 21432145

Pronovost PJ, Berenholtz SM, Needham DM (2008): Translating evidence into practice: a model for large scale knowledge translation. BMJ $\underline{337}$, a1714

Purrucker JC, Renzland J, Uhlmann L, Bruckner T, Hacke W, Steiner T, Bösel J (2015): Volatile sedation with sevoflurane in intensive care patients with acute stroke or subarachnoid haemorrhage using AnaConDa®: an observational study. Br J Anaesth 114, 934-943

Raabe A, Seifert V, Schmiedek P, Steinmetz H, Bertalanffy H, Steiger H-J, Stolke D, Forsting M (2002): Empfehlungen zum Management nichtrupturierter intrakranieller Aneurysmen. Cent Eur Neurosurg $\underline{63}, 70-76$

Rabinstein AA (2009): The AHA Guidelines for the Management of SAH: What we know and so much we need to learn. Neurocrit Care 10, 414

Rabinstein AA, Lanzino G, Wijdicks EF (2010): Multidisciplinary management and emerging therapeutic strategies in aneurysmal subarachnoid haemorrhage. Lancet Neurol $\underline{9}$, 504-519

Reiff T, Barthel O, Schönenberger S, Mundiyanapurath S (2020): High-normal PaCO2 values might be associated with worse outcome in patients with subarachnoid hemorrhage - a retrospective cohort study. BMC Neurol 20, 31

Reznik ME, Schmidt JM, Mahta A, Agarwal S, Roh DJ, Park S, Frey HP, Claassen J (2017): Agitation after subarachnoid hemorrhage: A frequent omen of hospital complications associated with worse outcomes. Neurocrit Care $\underline{26}, 428-435$

Riker RR, Fugate JE, Monitoring A the P in the IMCC on M (2014): Clinical monitoring scales in acute brain injury: Assessment of coma, pain, agitation, and delirium. Neurocrit Care 21, 27-37

Rinkel, Djibuti Mamuka, Algra Ale, van Gijn J. (1998): Prevalence and Risk of Rupture of Intracranial Aneurysms. Stroke 29, 251-256 
Rinkel GJE, Klijn CJM (2009): Prevention and treatment of medical and neurological complications in patients with aneurysmal subarachnoid haemorrhage. Pract Neurol $\underline{9}, 195-209$

Rossi S, Longhi L, Balestreri M, Spagnoli D, deLeo A, Stocchetti N (2000): Brain oxygen tension during hyperoxia in a swine model of cerebral ischaemia. Acta Neurochir Suppl $\underline{76}, 243-245$

Roux PL, Menon DK, Citerio G, Vespa P, Bader MK, Brophy G, Diringer MN, Stocchetti N, Videtta W, Armonda R et al. (2014): The International Multidisciplinary Consensus Conference on Multimodality Monitoring in Neurocritical Care: A list of recommendations and additional conclusions. Neurocrit Care 21, 282-296

Rowland MJ, Hadjipavlou G, Kelly M, Westbrook J, Pattinson KTS (2012): Delayed cerebral ischaemia after subarachnoid haemorrhage: looking beyond vasospasm. Br J Anaesth $\underline{109}$, 315-329

Rudolph U, Antkowiak B (2004): Molecular and neuronal substrates for general anaesthetics. Nat Rev Neurosci $\underline{5}, 709-720$

Sakowitz OW, Raabe A, Vucak D, Kiening KL, Unterberg AW (2006): Contemporary management of aneurysmal subarachnoid hemorrhage in Germany: Results of a survey among 100 neurosurgical departments. Neurosurgery $\underline{58}, 137-145$

Samaniego EA, Mlynash M, Caulfield AF, Eyngorn I, Wijman CAC (2011): Sedation confounds outcome prediction in cardiac arrest survivors treated with hypothermia. Neurocrit Care $\underline{15}$, 113-119

Sarrafzadeh AS, Vajkoczy P, Bijlenga P, Schaller K (2014): Monitoring in neurointensive care - the challenge to detect delayed cerebral ischemia in high-grade aneurysmal SAH. Front Neurol $\underline{5}$, 134

Sauvigny T, Mohme M, Grensemann J, Dührsen L, Regelsberger J, Kluge S, Schmidt NO, Westphal M, Czorlich P (2019): Rate and risk factors for a hyperactivity delirium in patients with aneurysmal subarachnoid haemorrhage. Neurosurg Rev $\underline{42}, 481-488$

Schmidt JM, Ko S-B, Helbok R, Kurtz P, Stuart RM, Presciutti M, Fernandez L, Lee K, Badjatia N, Connolly ES et al. (2011): Cerebral perfusion pressure thresholds for brain tissue hypoxia and metabolic crisis after poor-grade subarachnoid hemorrhage. Stroke $\underline{42}$, 1351-1356

Schmutzhard E, Beer R, Vajkoczy P (2010): Intensivmedizinische Therapie der aneurysmatischen Subarachnoidalblutung. Intensivmed Notfmed 47, 169-176

Sessler CN, Gosnell MS, Grap MJ, Brophy GM, O’Neal PV, Keane KA, Tesoro EP, Elswick RK (2002): The Richmond Agitation-Sedation Scale. Am J Respir Crit Care Med 166, 1338-1344

Seule MA, Stienen MN, Cadosch D, Fournier J-Y, Lussmann R, Hildebrandt G, Gautschi OP (2010): Aneurysmatische Subarachnoidalblutung - Diagnostik und Therapie zerebraler und systemischer Komplikationen. Anästhesiol Intensivmed Notfallmed Schmerzther 4토, 8-17

Skoglund K, Enblad P, Marklund N (2009): Effects of the neurological Wake-Up Test on intracranial pressure and cerebral perfusion pressure in brain-injured patients. Neurocrit Care $11,135-$ 142 
Skoglund K, Enblad P, Marklund N (2013): Monitoring and sedation differences in the management of severe head injury and subarachnoid hemorrhage among neurocritical care centers. J Neurosci Nurs $\underline{45}, 360-368$

Smith M (2007): Intensive care management of patients with subarachnoid haemorrhage. Curr Opin Anaesthesiol 20, 400-407

Steiner T, Juvela S, Unterberg A, Jung C, Forsting M, Rinkel G (2013): European Stroke Organization Guidelines for the management of intracranial aneurysms and subarachnoid haemorrhage. Cerebrovasc Dis $\underline{35}, 93-112$

Stevens RD, Naval NS, Mirski MA, Citerio G, Andrews PJ (2009): Intensive care of aneurysmal subarachnoid hemorrhage: an international survey. Intensive Care Med $\underline{35}, 1556-1566$

Teasdale G, Jennett B (1974): Assessment of coma and impaired consciousness: A Practical Scale. The Lancet $\underline{304}, 81-84$

The Brain Trauma Foundation (2000): Initial Management. J Neurotrauma 17, 463-469

Treggiari MM (2011): Hemodynamic management of subarachnoid hemorrhage. Neurocrit Care $\underline{15}$, 329-335

van Gijn J, Rinkel GJ (2001): Subarachnoid haemorrhage: diagnosis, causes and management. Brain $\underline{124}, 249-278$

van Gijn J, Kerr RS, Rinkel GJ (2007): Subarachnoid haemorrhage. The Lancet $\underline{369}$, 306-318

Villa F, Iacca C, Molinari A, Giussani C, Aletti G, Pesenti A, Citerio G (2012): Inhalation versus endovenous sedation in subarachnoid hemorrhage patients: Effects on regional cerebral blood flow. Crit Care Med 40, 2797-2804

Vlak MH, Algra A, Brandenburg R, Rinkel GJ (2011): Prevalence of unruptured intracranial aneurysms, with emphasis on sex, age, comorbidity, country, and time period: a systematic review and meta-analysis. Lancet Neurol 10, 626-636

von der Brelie C, Seifert M, Rot S, Tittel A, Sanft C, Meier U, Lemcke J (2017): Sedation of patients with acute aneurysmal subarachnoid hemorrhage with ketamine is safe and might influence the occurrence of cerebral infarctions associated with delayed cerebral ischemia. World Neurosurg 97, 374-382

Wermer M, van der Schaaf I, Algra A, Rinkel GJ (2007): Risk of rupture of unruptured intracranial aneurysms in relation to patient and aneurysm characteristics. Stroke $\underline{38}, 1404-1410$

Westermaier T, Stetter C, Kunze E, Willner N, Holzmeier J, Kilgenstein C, Lee J-Y, Ernestus R-I, Roewer N, Muellenbach RM (2014): Controlled transient hypercapnia: a novel approach for the treatment of delayed cerebral ischemia after subarachnoid hemorrhage?: Clinical article. J Neurosurg 121, 1056-1062

Westermaier T, Stetter C, Kunze E, Willner N, Holzmeier J, Weiland J, Koehler S, Lotz C, Kilgenstein C, Ernestus RI et al. (2016): Controlled hypercapnia enhances cerebral blood flow and brain tissue oxygenation after aneurysmal subarachnoid hemorrhage: Results of a phase 1 study. Neurocrit Care 25, 205-214 
Wilson J, Gelb A (2002): Free radicals, antioxidants, and neurologic injury: Possible relationship to cerebral protection by anesthetics. J Neurosurg Anesthesiol 14, 66-79

Yan K, Pang L, Gao H, Zhang H, Zhen Y, Ruan S, Wu W, Xu W, Gong K, Zhou X, Na H (2018): The influence of sedation level guided by bispectral index on therapeutic effects for patients with severe traumatic brain injury. World Neurosurg 110, 671-683

Yokoyama S, Hifumi T, Okazaki T, Noma T, Kawakita K, Tamiya T, Minamino T, Kuroda Y (2018): Association of abnormal carbon dioxide levels with poor neurological outcomes in aneurysmal subarachnoid hemorrhage: a retrospective observational study. J Intensive Care $\underline{6}$, 83

Zhao B, Fan Y, Xiong Y, Yin R, Zheng K, Li Z, Tan X, Yang H, Zhong M (2016): Aneurysm rebleeding after poor-grade aneurysmal subarachnoid hemorrhage: Predictors and impact on clinical outcomes. J Neurol Sci $\underline{371}$, 62-66 


\section{Danksagung}

Der größte Dank gilt meinem Doktorvater und Betreuer dieser Arbeit, Herrn Priv. Doz. Dr. Christian von der Brelie.

Desweiteren möchte ich mich herzlich für die Unterstützung bei folgenden Personen bedanken, meinem Zweitbetreuer Herrn Prof. Dr. José Hinz, der Erstautorin des korrespondierenden Papers Frau Dr. Silvia Hernandéz- Durán, sowie Herrn Prof. Dr. J. Schaeper, Herrn Prof. Dr. O. Moerer, Herrn Prof. Dr. V. Rohde, Frau Prof. Dr. D. Mielke für die Co-Autorenschaft des Papers „Mechanical ventilation, sedation and neuromonitoring of patients with aneurysmal subarachnoid hemorrhage in Germany: Results of a nationwide survey“. Herrn Dr. Golinski danke ich für das Feedback zum Erstellen des Fragebogens. Desweiteren danke ich den Mitarbeitern des Instituts für medizinische Statistik in Göttingen für die freundliche Beratung und Unterstützung hinsichtlich der Auswertung der Ergebnisse.

Zu guter Letzt sei an dieser Stelle ein großer Dank allen Zentren ausgesprochen, die an der Umfrage teilgenommen haben, ohne deren Kooperation die Daten für diese Arbeit nicht hätten erhoben werden können. 
